\title{
Optogenetic Manipulation of the Auditory System
}

\author{
Dissertation \\ for the award of the degree \\ "Doctor rerum naturalium" \\ of the Georg-August-Universität Göttingen
}

within the doctoral program

"International Max Planck Research School for Neuroscience"

of the Georg-August University School of Science (GAUSS)

submitted by

Burak Bali

from Erzurum, Turkey 


\section{Thesis Committee and Members of the Examination Board}

\section{Referee: Prof. Dr. Tobias Moser}

Institute for Auditory Neuroscience, University Medical Center Göttingen

$2^{\text {nd }}$ Referee: Prof. Dr. Manuela Schmidt

Somatosensoy Signaling Group, Max Planck Institute for Experimental Medicine

(if applicable) $3^{\text {rd }}$ referee: Dr. Jens Gruber

Medical RNA Biology, German Primate Center

\section{Further members of the Examination Board}

Arezoo Pooresmaeili, MD, PhD

Perception and Cognition, European Neuroscience Institute

Prof. Dr. Tim Gollisch

Sensory Processing in the Retina, University Medical Center Göttingen

Prof. Dr. Alexander Flügel

Institute for Neuroimmunology and Multiple Sclerosis Research, University Medical Center Göttingen

Date of oral examination: 15.01.2021 
Herewith I declare that I prepared this doctoral dissertation, entitled "Optogenetic Manipulation of the Auditory System", on my own and with no other sources and aids than quoted.

Burak Bali

Göttingen, 30 November 2020 


\section{Table of Contents}

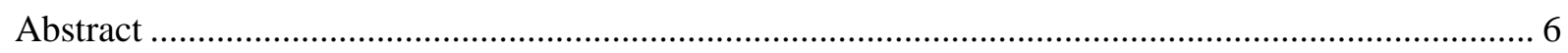

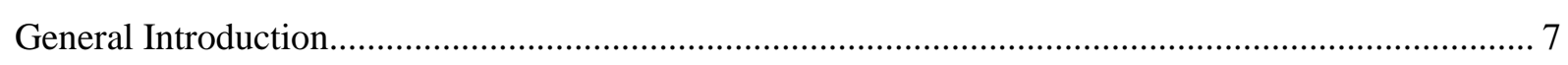

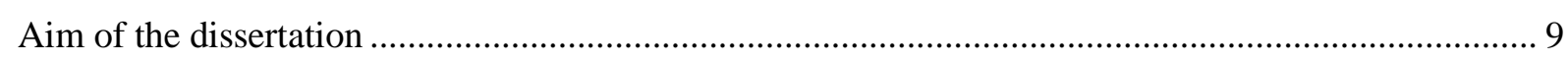

Chapter 1: Ultrafast optogenetic stimulation of the auditory pathway by targeting-optimized Chronos

10

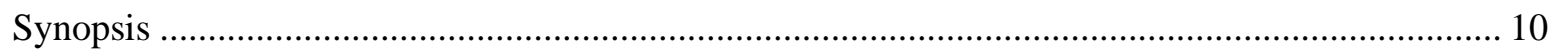

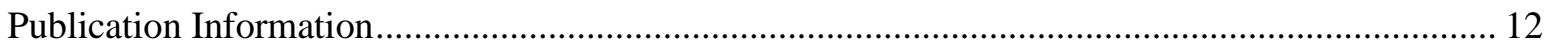

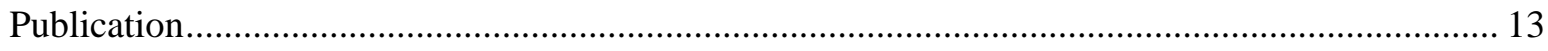

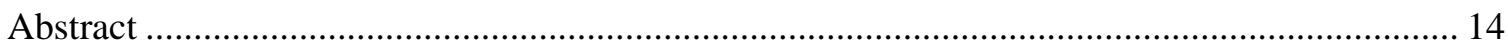

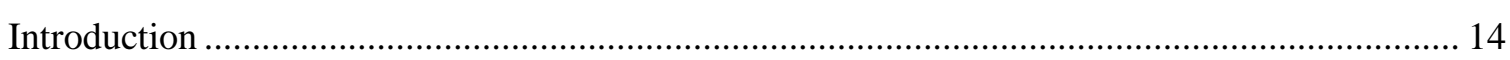

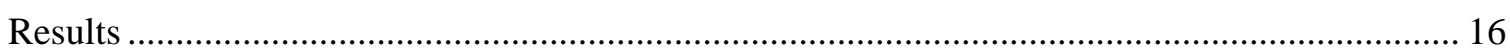

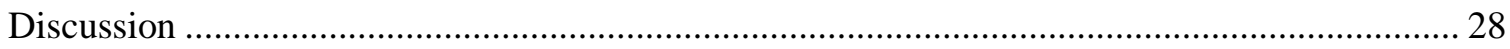

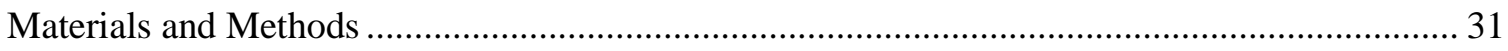

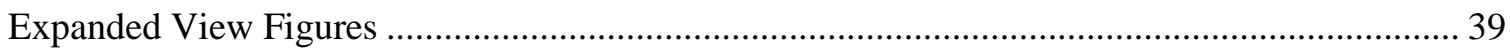

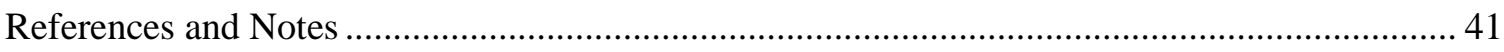

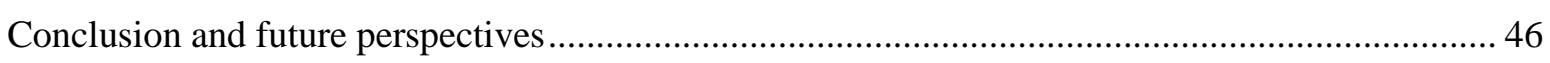

Chapter 2: Utility of red-light ultrafast optogenetic stimulation of the auditory pathway .................... 47

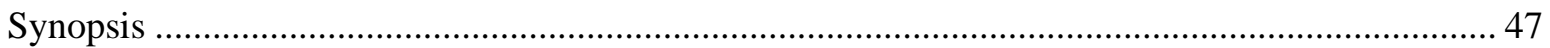

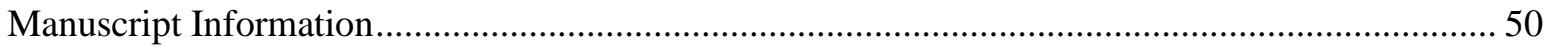

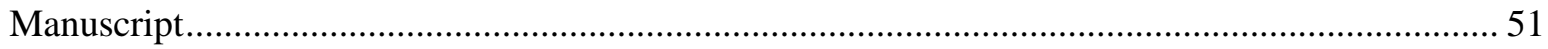

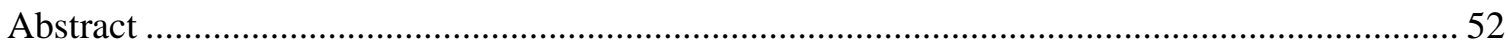

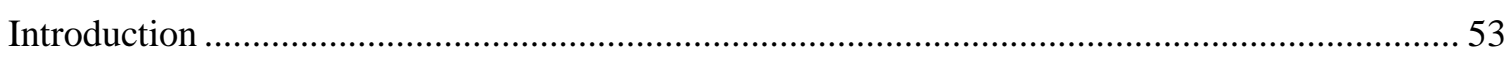

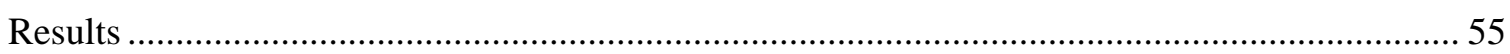

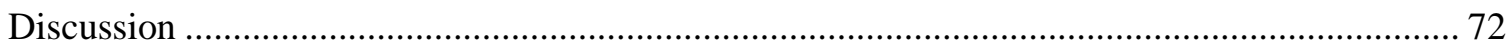

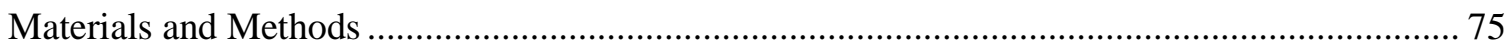

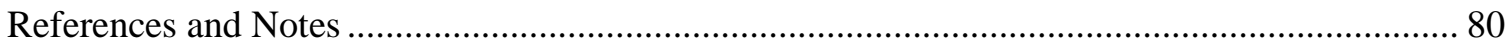

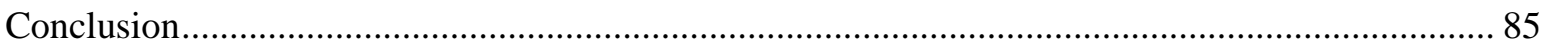

Chapter 3: Nonclinical biosafety assessment of red-shifted cochlear optogenetics ............................. 86

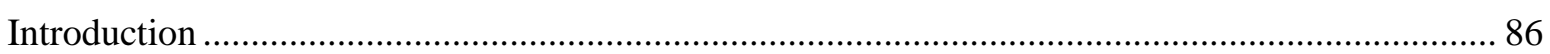

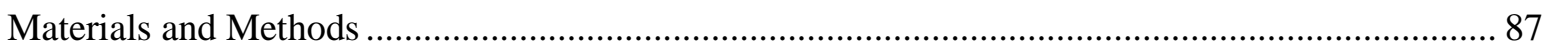

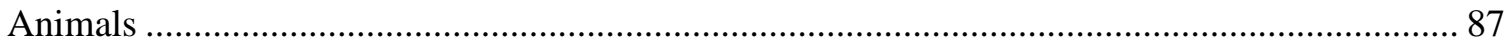

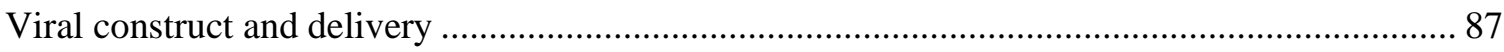

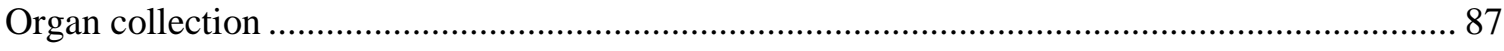

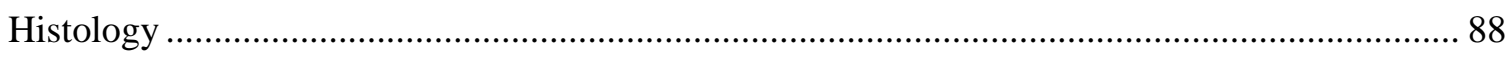

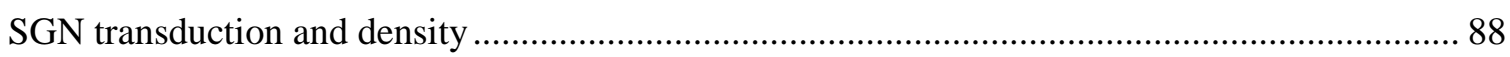

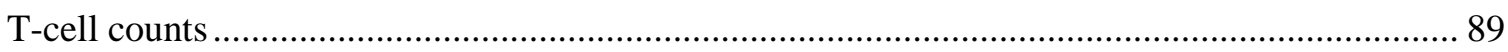




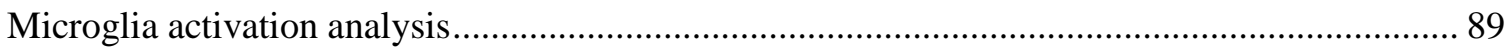

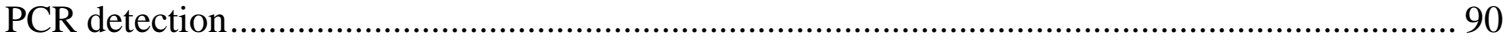

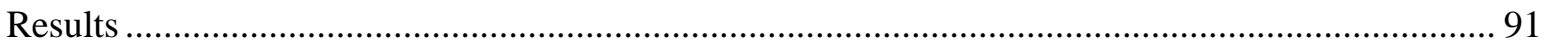

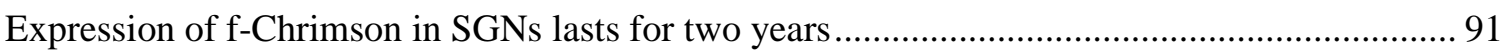

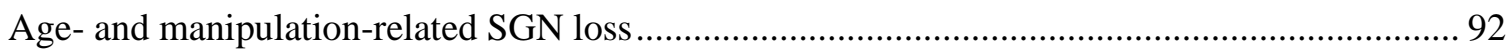

Spread of f-Chrimson expression to the brain following postnatal AAV-injection ....................... 95

Inflammatory traces in brain are age-related rather than due to AAV spread ................................. 97

Viral f-Chrimson construct was not detected in inner organs ................................................... 102

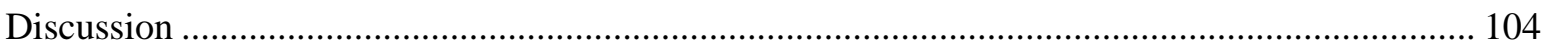

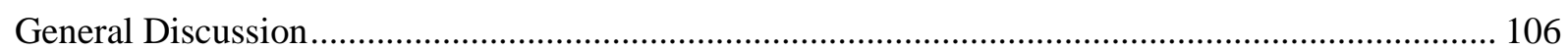

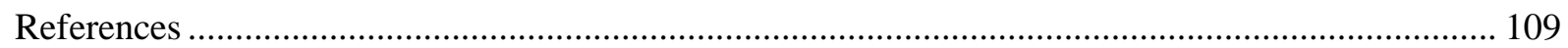

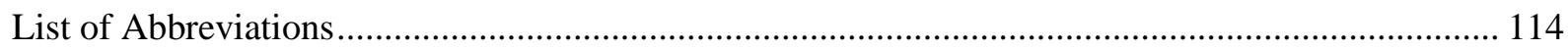

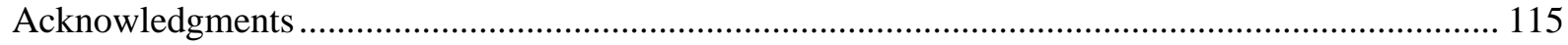

Curriculum Vitae ..................................................................................Error! Bookmark not defined. 


\section{Abstract}

There are millions of people who are affected from sensorineural hearing loss. In this type of deafness, sensory hair cells are typically dysfunctional or lost, and thereby not conveying sound information to brain. The state-of-the-art solution is the cochlear implant (CI) which circumvents the receptor problem via direct electric stimulation of spiral ganglion neurons (SGNs) of that from auditory nerve (AN). Thus, the CI enables hearing and provides open speech comprehension. Yet, for instance in noisy background, in most users, speech comprehension fails likely owing to poor frequency resolution. This results from wide current spread in the the saline-filled cochlea activating large sets of the tonotopically organized SGNs. Here, light can serve as an alternative mode of stimulation since it can be conveniently focused to evoke SGN populations more precisely. Clearly, SGNs are not light sensitive and hence we employ an optogenetic approach. The series of studies presented here gathers under the same roof: How to achieve optogenetic manipulation of the SGNs such that it will suit well to the intrinsic physiological properties of auditory system, be stably maintained for many years, and last but not least be safe? Temporal firing properties of SGNs are uniquely fast allowing fine coding of sound. Therefore, channelrhodopsins (ChRs) used should enable reliable control of light-induced spiking in SGNs. To this end, first, we investigated feasibility of blue-light-gated naturally occurring Chronos with the fastest on-/off-kinetics at the mouse AN with the help of molecular tools, i.e. endoplasmic reticulum exit signal (ES) and membrane trafficking signal (TS), enhancing membrane localization of the ChR. By this way, it was possible to drive the AN up to $1000 \mathrm{~Hz}$ stimulation rate. While Chronos suits temporal coding very well, we also sought for red-shifted fast alternatives since chronic exposure to blue light bears risk of phototoxicity in SGNs. Here, the engineered red-shifted very-fastChrimson (vf-Chrimson-ES/TS) which has similar fast kinetics as fast as Chronos-ES/TS, evoked fast responses until $500 \mathrm{~Hz}$ stimulation rate from the mouse AN. Moreover, we further characterized light intensity encoding of vf-Chrimson-ES/TS in comparison to the slower variant fast-Chrimson (f-Chrimson). Third, we studied the long-term availability and biosafety profile of f-Chrimson, a prominent candidate for use with the optical CI, showing expression in SGNs up to two years following early postnatal AAV-based delivery to the inner ear. There was also a tendency of SGN loss due to combinatorial effect of aging and fChrimson expression. Expression spread to the non-injection ear and parts of the brain. The spread was restricted to the vicinity of injection and was not detected in the distal organs such as kidney and spleen. All in all, these three projects that I was involved in at different extents pave the way to future medical optogenetic cochlear implants. 


\section{General Introduction}

The auditory system enables us to understand our daily acoustic scene such as speech, music, or click-clacks of my keyboard while typing these words. Upon arrival to the outer ear, composed of pinnae and auditory canal, these airborne soundwaves are funneled down to the eardrum, the thin tissue membrane bordering outer ear from the middle ear. It translates airtraveling soundwaves to mechanical vibrations and conveys the sound information to the middle ear residing auditory ossicles, malleus, incus and stapes which further transmits these vibrations to the place where neural coding of sound starts - the cochlea of the inner ear.

The snail-shaped bony structure of the inner ear is called the cochlea. It is composed of three fluid-filled chambers, i.e. (1) scala vestibuli and (2) scala tympani with perilymph, and (3) scala media with endolymph. Scala media is separated from the former two by Reissner's and basilar membranes, respectively. More importantly, it houses the organ of Corti, the neural mechanoelectric transformer of sound, which sits on the basilar membrane. Journey of the soundwave here continues through cochlear labyrinth and activates specific regions according to its frequency. This place code of cochlea, or tonotopy, is shaped by the physical properties of the basilar membrane which is highly stiff and thin at the base so that it vibrates most to the high frequency sounds. On contrary, this membrane is less stiff and thicker at the apex of the cochlea where then low frequency sounds are detected (von Bekesy and Wever, 1960; Oghalai, 2004). Sensory cells of the auditory system, inner and outer hair cells (IHCs and OHCs), residing in the organ of Corti are then activated and already starts coding for sound along the tonotopic axis. IHCs are responsible for conveying sound information to the auditory nerve whereas OHC amplify and sharpen the soundwaves (Ashmore, 2008).

In classical neuroanatomy, cranial nerve VIII is composed of vestibular and cochlear nerves leaving from mammalian inner ear (Carricondo and Romero-Gomez, 2019). The former has a role in body balance. The latter is also known as auditory nerve (AN) which is a bundle of afferent and efferent axon fibers relaying and modulating acoustic information. The afferent auditory nerve fibers (ANFs) are the central axons of primary bipolar spiral ganglion neurons (SGNs) which functionally are categorized into two: type I SGNs receiving information from the IHCs and type II from the OHCs. Type I SGNs are the majority constituent ( 90-95 \%), that have one-to-one connections to the tonotopically-organized inner hair cells. Therefore, they are also already tuned to the frequency of sound stimuli. Here, spike rate per SGN and number of SGNs recruited play a role in coding for sound amplitude (Dieter et al, 2020). 
When hair cells are dysfunctional or lost, current way to rehabilitate hearing is via electric cochlear implants (eCIs). The eCI is a medical device having two parts being external and internal. The former part is composed of a microphone and a processor which picks up the incoming sounds and analyzes frequency information of them and activates the internal part accordingly. Internal part of the eCI is linearly placed electrodes which span the tonotopic axis of the cochlea. Depending on the frequency of sound, electrodes inject current on to the SGNs in their vicinity to trigger spiking. Thus, for most users, open speech comprehension is provided. Yet, larger populations of SGNs are activated due to broad current spread from each active electrode where spectral resolution of sound is reduced (Shannon, 1983). This, for example, hampers understanding speech on a background noise for eCI users (Caldwell et al, 2017). 


\section{Aim of the dissertation}

In the course of developing an optogenetic cochlear implant for human use, the ultimate goal is to reinstate lost hearing function as natural as possible. This light-driven system should primarily refine spectral resolution of sound encoding which is limited in electrical CIs. However, while better utilizing the cochlear place code of frequency, optical stimulation of the auditory nerve should not lose out on exact coding of temporal features. In our optogenetic approach, the activation/deactivation cycle time of channelrhodopsins represents the limiting factor. Once the optogenetic hearing restoration is established, it should function for many years without harm. Therefore, preclinical testing of the optogenetic approach, i.e. genetic modification and to light stimulation need to evaluate stability and biosafety before human use. Here, my thesis elaborates making of an optogenetic cochlear implant system at three tiers:

1. by characterizing optical response properties of mouse auditory nerve using ultrafast opsin Chronos after membrane trafficking enhancement,

2. By investigating utility of ultrafast and red-shifted channelrhodopsins, fast- and very-fast-Chrimson, for future cochlear optogenetic applications,

3. By evaluating longevity, spread and possible effects of viral delivery of fastChrimson into the mouse cochlea. 


\section{Chapter 1: \\ Ultrafast optogenetic stimulation of the auditory pathway by targeting-optimized Chronos}

\section{Synopsis}

Understanding temporal features of neural coding is a prominent step to decipher how the nervous system works. The auditory system is exemplary to study temporal neural coding as the acoustic stimuli are computed within few milliseconds to result in a percept/behavior. Already at the auditory nerve, in mammals for example, the fibers can resolve temporal fine structures of a low-frequency pure tone $(\leq 4-5 \mathrm{kHz})$ by firing action potentials synchronous to the preferred phase of the stimulus cycle. This so-called phase-locked response behavior of auditory nerve fibers is thought to play a role in localizing sounds, perceiving low pitch and speech comprehension in normal hearing (Smith et al., 2002). In case of hearing via cochlear implant, as brief electric pulses $(80 \mu \mathrm{s})$ are used to encode the sound pressure envelop at rates of hundreds to thousands of hertz. However, increasing stimulation rate beyond $500 \mathrm{~Hz}$, does not lend to a better pitch perception for CI users (Shannon et al., 2011), possibly due to the current spread and charge accumulation in the cochlea. In addition to synchronicity of firing, the AN fibers show intrinsically high firing rates in particular at sound onset where discharge rates $\geq 1000$ spikes per second are observed (Taberner and Liberman, 2005). Although the firing rate drops to a sustained rate of 200-400 spikes per second due to the adaptation to the tone, fibers of the SGNs are still one of the fastest spiking constituents of the nervous system.

Over years, several optogenetic tools have been discovered and/or engineered to study lightcontrolled fine-grade temporal coding in neurons (Han et al., 2009). Among the characteristics of channelrhodopsins like action spectrum, channel conductance, expression and membrane localization, it is the channel on-/off-kinetics that primarily governs the temporal precision of optogenetic stimulation (Lin et al., 2011). Generally, opening of the channelrhodopsins are instantaneous roughly relative to light stimulus onset. The limiting time constant is then bound to closing kinetic ( $\tau$ off) which varies vastly across opsins. Classical ChR2 for example, has a closing rate of $\sim 10 \mathrm{~ms}$ at room temperature; whereas, redshifted VChR1 needs more than $90 \mathrm{~ms}$ to undergo into the closed state, and some mutants of ChR2 can remain open in the range of minutes (Jeschke and Moser, 2015). In different neuron cultures, the light cycle of ChR2 allowed to reliably produce spikes with using light pulse trains up to $30 \mathrm{~Hz}$ stimulation rate (Arenkiel et al., 2007; Boyden et al., 2005). Considering much faster spiking AN fibers, for faithful optical coding of sound, one should also ensure 
fast and high-fidelity control through optogenetics where such slow ChR kinetics will prove to be insufficient.

Besides fast ChR gating, for reliable command of spike timing and discharge, several studies emphasized the importance of efficient localization of channelrhodopsins to the neuronal cell membrane (Garita-Hernandez et al., 2018; Gauvin et al., 2020; Gradinaru et al., 2014). When expressed in mammalian cells, microbial opsins, evolved to function most efficiently in protozoans, may not have compatible signaling peptides which forward them to the membrane. Therefore, despite being trafficked to cell membrane, opsins can still be found abundantly in intracellular compartments such as membranous endoplasmic reticulum and the Golgi apparatus. To enhance membrane localization of channelrhodopsins, fluorescent tags are thought to have a helper role (Gauvin et al., 2020) or trafficking signals, ER export signal (ES) and membrane trafficking signal (TS), from the mammalian potassium channels Kir2.1 and Kir2.4 (Hofherr et al., 2004; Stockklausner et al., 2001) can be added.

In 2014, Klapoetke and colleagues described a novel channelrhodopsin, named Chronos, having the fastest off-kinetic which still holds true today. This naturally occurring blue-lightgated opsin, from the algae S.helveticum, has a $\tau$ off of $3.6 \mathrm{~ms}$ on average at room temperature which could drop to submillisecond range at $35 \mathrm{oC}$. This would suggest that this new opsin can enable generating precisely-timed spike series at high stimulation rates. In hippocampal neuron culture, Chronos compared ChR2 permitted action potentials with high probability and fidelity at $60 \mathrm{~Hz}$ stimulation rate even at lower illumination. Despite providing a sturdy proof of concept, to be able to test its full potential, one requires an inherently fast-spiking neuron type - spiral ganglion neurons.

In the following research article, we investigated electrophysiological characterization of Chronos-mediated photocurrents in vitro and SGN spiking in vivo to elaborate its capacity and to evaluate its potential for a future oCI. As the first step, in HEK cells, Chronos is shown to allow ultrafast light-induced currents at $36 \mathrm{oC}$. Then, the focus shifts to step-by-step enhancement of expression and membrane localization of the opsin to be able to elicit reliable responses from the auditory nerve. For that, firstly the method of gene delivery is changed from in utero to postnatal injections. Then, the viral capsid used is switched from AAV2/6 to AAV-PHP.B. At the end, addition of the ES and TS sequences ensures membrane localization. Once the efficient targeted expression paradigm was set up, we were able to elicit ultrafast light-triggered physiological responses from the mouse auditory nerve. 
Publication Information

\section{Ultrafast optogenetic stimulation of the auditory pathway by targeting-optimized Chronos}

Keppeler, D. ., Martins Merino, R. , Lopez de la Morena, D.*, Bali, B* ., Huet, A.T.", Gehrt, A, Wrobel, C, Subramanian, S, Dombrowski, T., Wolf, F., Rankovic, V., Neef, A. and Moser, T.

*,\#These authors contributed equally to the work

EMBO J, Accepted manuscript

doi: 10.15252/embj.201899649

Detailed author contribution of Burak Bali:

- Experimental work and analysis:

- Confocal imaging and expression/density analysis of cochlear cryosections (Fig 4 C, D, F, G)

- Preparation of the manuscript (together with the other authors)

Copyright notice:

This material is reprinted by permission from EMBO Press in accordance to their copyright and licensing regulations. Reprinted by permission from Wiley-VCH Verlag GmbH \& Co. KGaA: () owned by the authors, 2018. 


\section{Publication}

\section{Ultrafast optogenetic stimulation of the auditory pathway by targeting-optimized Chronos}

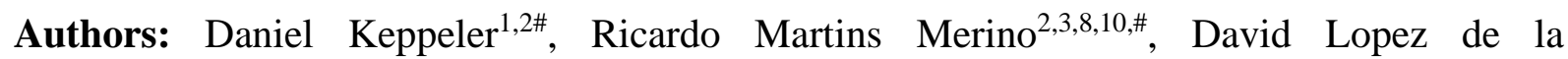
Morena $^{1,2,5 \#}$, Burak Bali ${ }^{1,2,6, \circ}$, Antoine Tarquin Huet ${ }^{1,3,5, \circ}$, Anna Gehrt ${ }^{1,7}$, Christian Wrobel ${ }^{1,7}$, Swati Subramanian ${ }^{1,2}$, Tobias Dombrowski ${ }^{1, \S}$, Fred Wolf ${ }^{4,7,8,10,11}$, Vladan Rankovic ${ }^{1,6^{*}}$, Andreas Neef $f^{3,8,11^{*}}$, Tobias Moser ${ }^{1,2,3,5,7,9,10^{*}}$

\section{Affiliations:}

${ }^{1}$ Institute for Auditory Neuroscience and InnerEarLab, University Medical Center Göttingen, Germany.

${ }^{2}$ Göttingen Graduate School for Neurosciences and Molecular Biosciences, University of Göttingen, Germany.

${ }^{3}$ Biophysics of Neural Computation Group, Bernstein Center for Computational Neuroscience Göttingen, Germany.

${ }^{4}$ Bernstein Center for Computational Neuroscience Göttingen, Germany.

${ }^{5}$ Auditory Neuroscience and Optogenetics Laboratory, German Primate Center, Göttingen, Germany.

${ }^{6}$ Restorative Cochlear Genomics group, Auditory Neuroscience and Optogenetics laboratory, German Primate Center, Göttingen, Germany.

${ }^{7}$ Collaborative Research Center 889, University of Göttingen, Göttingen, Germany.

${ }^{8}$ Neurophysics Group, Max Planck Institute for Dynamics and Self-Organization, Göttingen, Germany.

${ }^{9}$ Center for Nanoscale Microscopy and Molecular Physiology of the Brain, Göttingen, Germany.

${ }^{10}$ Max Planck Institute for Experimental Medicine, Göttingen, Germany.

${ }^{11}$ Campus Institute for Dynamics of Biological Networks, Göttingen, Germany.

$\#,{ }^{\circ}$ equal contribution

${ }^{\S}$ Department of Otorhinolaryngology, Head and Neck Surgery, Ruhr University Bochum, St. Elisabeth Hospital, Bochum, Germany.

*To whom correspondence should be addressed:

Dr. Vladan Rankovic; Dr. Andreas Neef; Prof. Dr. Tobias Moser. 


\begin{abstract}
Optogenetic tools, providing non-invasive control over selected cells, have the potential to revolutionize sensory prostheses for humans. Optogenetic stimulation of spiral ganglion neurons (SGNs) in the ear provides a future alternative to electrical stimulation used in cochlear implants. However, most channelrhodopsins do not support the high temporal fidelity pertinent to auditory coding because they require milliseconds to close after light-off. Here, we biophysically characterized the fast channelrhodopsin Chronos and revealed a deactivation time constant of less than a millisecond at body temperature. In order to enhance neural expression, we improved its trafficking to the plasma membrane (Chronos-ES/TS). Following efficient transduction of SGNs using early postnatal injection of the adenoassociated virus AAV-PHP.B into the mouse cochlea, fiber-based optical stimulation elicited optical auditory brainstem responses (oABR) with minimal latencies of $1 \mathrm{~ms}$, thresholds of $5 \mu \mathrm{J}$ and $100 \mu$ s per pulse, and sizable amplitudes even at $1000 \mathrm{~Hz}$ of stimulation. Recordings from single SGNs demonstrated good temporal precision of light-evoked spiking. In conclusion, efficient virus-mediated expression of targeting-optimized Chronos-ES/TS achieves ultrafast optogenetic control of neurons.
\end{abstract}

\title{
Introduction
}

Since the discovery of channelrhodopsins (ChRs, (Nagel et al., 2002, 2003) and the application of these light-gated ion channels for controlling excitable cells (Boyden et al., 2005), the concept of optogenetics has revolutionized the life sciences (Adamantidis et al., 2015; Kim et al., 2017). Application of optogenetics to restore sensory function in the immune-privileged eye and the ear are thought to have a fair chance of clinical translation (Jeschke and Moser, 2015; Sahel and Roska, 2013). Indeed, AAV-mediated optogenetics for vision restoration has recently entered a first clinical trial in a dose-finding effort (ClinicalTrials.gov, 2015; Identifier: NCT02556736) while neural coding of visual information can likely be achieved with $\mathrm{ChRs}$ that deactivate within several milliseconds (Busskamp et al., 2012), faster ChRs are required for sound coding in spiral ganglion neurons of the ear (SGNs) that spike at hundreds of $\mathrm{Hz}$ with sub-millisecond precision (Jeschke and Moser, 2015).

If such ultrafast optogenetic control of neural activity was available, it would serve auditory research and could fuel the development of future optical cochlear implants (oCIs). Clinically, this is highly relevant as approximately 466 million people-over 5\% of the world's 
population-suffer from a disabling hearing impairment (WHO, 2018) and we are still lacking causal therapies for the most common form; sensorineural hearing impairment. Consequences are impaired communication, often social isolation, depression, and reduction in professional capabilities. As of today, partial restoration of auditory function by hearing aids and electrical CIs (eCI) represent the options of choice for rehabilitation in sensorineural hearing impairment, which results from cochlear dysfunction or degeneration. The eCI bypasses dysfunctional or lost cochlear hair cells via direct electric stimulation of SGNs and, with most of the approximately 500.000 users achieving open speech comprehension, is considered the most successful neuroprosthesis (Lenarz, 2018; Zeng, 2017). Nonetheless, there is an urgent need for further improvement of the CI. The biggest bottleneck of the eCI is the poor spectral resolution of coding that arises from the wide spread of current around each electrode contact (Kral et al., 1998). Using light for stimulation in oCI is one of the present developments to improve spectral coding by CIs, as light can be better spatially confined than electric current (e.g. Richter et al, 2011; Hernandez et al, 2014). One of the implementations used optogenetic stimulation of SGNs for a first proof-of-principle study on activation of the auditory pathway up to the midbrain (inferior colliculus, IC), demonstrating a lower spread of cochlear excitation for fiber-based oCI than for monopolar eCI (Hernandez et al., 2014a). However, the temporal fidelity of ChR2-mediated optogenetic control of SGN firing seemed limited; auditory brainstem responses broke down even below $100 \mathrm{~Hz}$ of stimulation. Higher temporal fidelity of optogenetic SGN stimulation might be achieved when using faster ChRs such as Chronos (Klapoetke et al., 2014), the newly engineered Chronos mutant ChroME (Mardinly et al., 2018) or fast Chrimson mutants (Mager et al., 2018).

Therefore, characterizing and optimizing fast ChRs is of great importance for fast spiking neurons in the auditory system, but also in the somatosensory system, cerebellum, and a wide range of inhibitory circuits. Here, we targeted Chronos, the fastest ChR reported so far, and first dissected its gating by patch-clamp recordings of photocurrents. Towards its application for optogenetic stimulation of mouse SGNs we optimized Chronos, the viral vector and virus injection approach for achieving high plasma membrane expression. As described for another opsin (Gradinaru et al., 2010) we appended sequences for improved exit from the endoplasmic reticulum (ES) (Ma et al., 2001; Stockklausner et al., 2001) and trafficking to the plasma membrane (TS) (Hofherr, 2005) to Chronos (Chronos-ES/TS) and performed postnatal injections of AAV-PHP.B serotype (Deverman et al., 2016), which drove highly efficient Chronos-ES/TS expression. We demonstrate by recordings of oABR and single SGN firing that Chronos-ES/TS enables ultrafast stimulation of the auditory pathway. 


\section{Results}

Chronos undergoes sub-millisecond on/off transitions at $36^{\circ} \mathrm{C}$

The kinetic properties of ChRs are best studied in cells with little background conductance to characterize the light-induced conductance in isolation. To this end, we used human embryonic kidney cells 293T (HEK-293T cells) expressing Chronos or ChR2 (Methods). We clamped the membrane voltage to $-60 \mathrm{mV}$ where any light-induced change in the conductance is then linearly reflected in a change of the pipette current. First, we compared gating kinetics of ChR2 and Chronos at a low light intensity of $0.27 \mathrm{~mW} \mathrm{~mm}^{-2}$, far below the half maximal activation. At $22^{\circ} \mathrm{C}$, we found activation and de-activation time constants (mean \pm s.e.m.) of $\tau_{\text {act }}=4.9 \pm 0.3 \mathrm{~ms}, \quad \tau_{\text {deact }}=9.4 \pm 1.0 \mathrm{~ms} \quad(\mathrm{n}=6) \quad$ for $\quad \mathrm{ChR} 2$ and $\tau_{\text {act }}=1.5 \pm 0.1 \mathrm{~ms}$, $\tau_{\text {deact }}=3.0 \pm 0.2 \mathrm{~ms}(\mathrm{n}=21)$ for Chronos (Figure 1.1A). When increasing the temperature to $36^{\circ} \mathrm{C}$, activation and deactivation accelerated, with $\tau_{\text {act }}=0.58 \pm 0.02 \mathrm{~ms}$ and $\tau_{\text {deact }}=0.76 \pm 0.05 \mathrm{~ms}(\mathrm{n}=6)$, Chronos reached the sub-millisecond range, while ChR2 gating kinetics, $\tau_{\text {act }}=2.3 \pm 0.1 \mathrm{~ms}, \tau_{\text {deact }}=3.0 \pm 0.3 \mathrm{~ms}$ ( $\mathrm{n}=6$ and 5 respectively), at $36^{\circ} \mathrm{C}$ were comparable to the values achieved by Chronos at $22^{\circ} \mathrm{C}$. Hence, already at such low light intensity, Chronos, but not ChR2, activation and deactivation should permit signal transmission with a bandwidth of several hundred Hertz. Probing the frequency bandwidth directly by applying light chirps, Chronos confers a much higher bandwidth compared to ChR2 (Figure 1.1B). The gain of chirp responses could be very well characterized by a single cut-off frequency of $24 \mathrm{~Hz}$ and $86 \mathrm{~Hz}$ for $\mathrm{ChR} 2$ and Chronos, respectively, at $22^{\circ} \mathrm{C}$ and $63 \mathrm{~Hz}$ and $150 \mathrm{~Hz}$ at $36^{\circ} \mathrm{C}$ (Figure $1.1 \mathrm{C}$ ).

\section{Improving the plasma membrane expression of Chronos}

The above biophysical characterization had indicated Chronos as a strong candidate for optogenetic stimulation of SGNs with the required high temporal fidelity, provided sufficient plasma membrane expression can be achieved. Recent studies have shown that adding ER export and trafficking signals, isolated from a vertebrate inward rectifier potassium channel, to the cytoplasmic C-terminus of opsins promote their plasma membrane expression (Gradinaru et al., 2010). Hence, we added these sequences, here nick-named ES (Export Signal) and TS (Trafficking Signal), sandwiching EYFP, to Chronos (Chronos-ES/TS, Figure 1.2A). We first compared expression of Chronos-ES/TS and Chronos in HEK-293T cells transfected with the respective pAAV plasmid by immunocytochemistry. Using confocal and stimulated emission depletion (STED) microscopy we found a preferentially peripheral, likely plasmalemmal, localization of Chronos-ES/TS with some intracellular signal most likely 
arising from the Golgi, while the original Chronos construct was more diffusely distributed throughout the extra-nuclear intracellular space (Figure 1.2B-D). We quantified the cellular distribution of the opsin by line profile analysis (Figure 1.2C) and found a significantly greater peripheral than central immunofluorescence for Chronos-ES/TS when compared to the original Chronos construct (Figure 1.2C, D). The larger variance of the ratio of membrane and intracellular fluorescence for Chronos-ES/TS (Figure 1.2D) is likely explained by dividing by the relatively low, yet varying intracellular abundance of the opsin. In summary, the data suggests improved trafficking to the plasma membrane of Chronos-ES/TS.
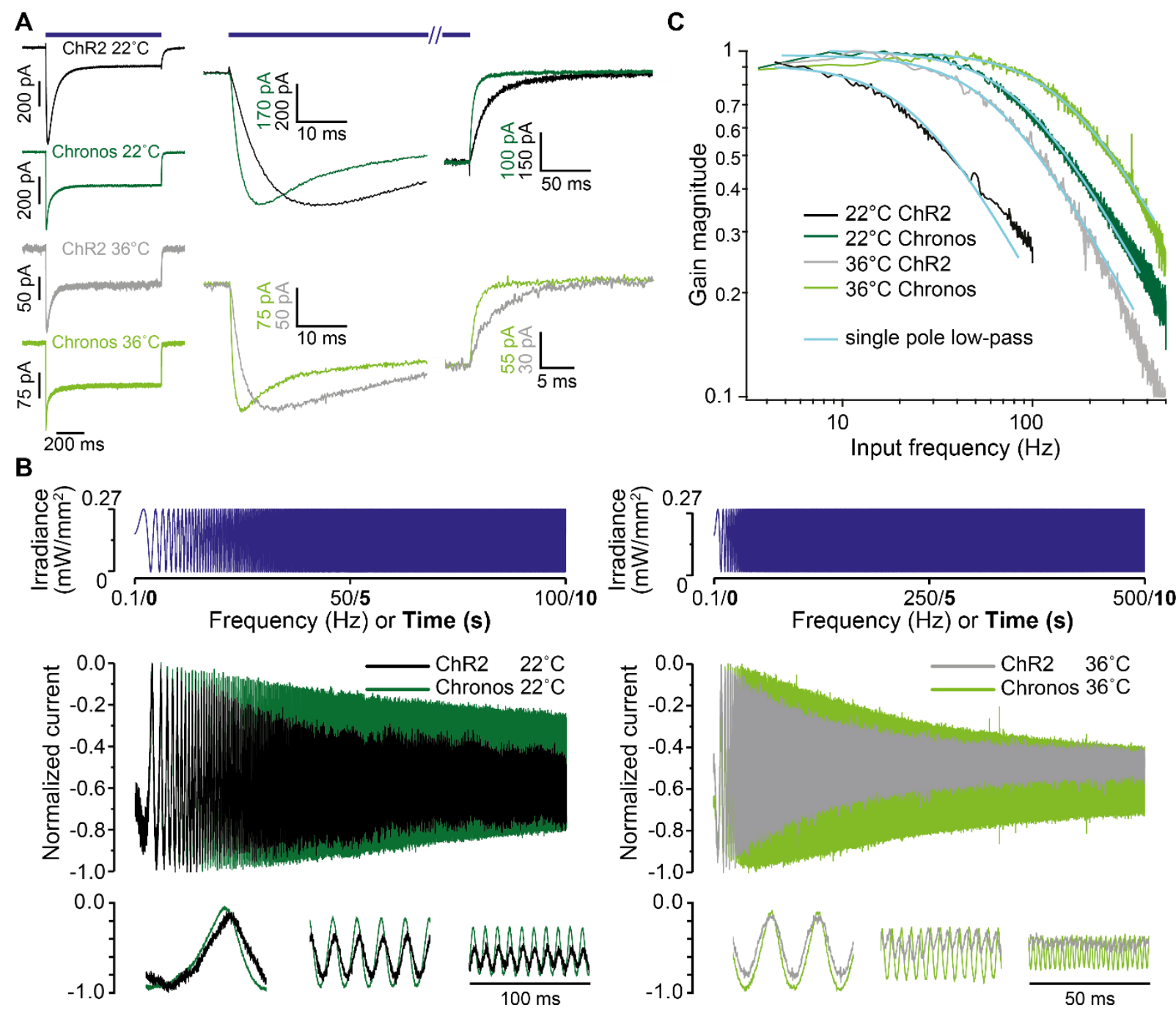

Figure 1.1: Chronos mediates fast light-driven currents

A: One second long light pulses (LED centered $480 \mathrm{~nm}, 0.27 \mathrm{~mW} \mathrm{~mm}^{-2}$ ) elicit current responses in HEK-293T cells expressing ChR 2 and Chronos, tested at $22^{\circ} \mathrm{C}$ and $36^{\circ} \mathrm{C}$. Right panels show activation and deactivation at higher time resolution. B: A quasi-periodic chirp stimulus is used to directly probe the bandwidth of Chronos- and ChR2-mediated photocurrents in HEK-293T cells. Top: stimulus, middle: full response, bottom: sections from the beginning, middle and end of the response. Note the substantially larger frequency range over which Chronos currents follow the light stimulus. At $36^{\circ} \mathrm{C}$ this range is extended even further. C: Analysis of the chirp responses of HEK-293T cells as in B. The current amplitude 
modulation is plotted against the stimulus frequency. The smooth lines represent fits to the magnitude of the transfer function of a single pole filter $a b s\left(\left(1+i f / f_{c u t}\right)^{-1}\right)$.

Similar findings were also obtained in hippocampal neurons in culture transduced by AAV2/6 or AAV-PHP.B (Figure 1.3). This indicates that neurons, too, struggle to traffic Chronos to the plasma membrane and that this can be alleviated when adding the ES- and TS-signals, at least when tested in culture.

\section{AAV-mediated expression of Chronos and Chronos-ES/TS in mouse SGNs}

Next, we turned to expression of Chronos in mouse SGNs in vivo and, once more, compared the original Chronos and Chronos-ES/TS. We aimed to establish efficient AAV-mediated transduction of SGNs and employed the human synapsin promoter (hSyn, Figure 1.2A) that had turned out to drive efficient and specific SGN expression (Hernandez et al., 2014a). We first followed our previous protocol using transuterine injections of AAV2/6 into the otocyst of mouse embryos at embryonic day 11.5 (Figure 1.4A, upper). In most of the cases the expression of Chronos-EGFP was absent or sparse (Figure 1.4B, left and middle). Exceptionally we saw high expression levels (Figure 1.4B right). As before (Hernandez et al., 2014a), the expression, if any, was largely limited to the SGNs of the basal cochlear turn and was never seen in inner hair cells (Figure 1.4B right, inset).

Next, we moved on to early postnatal injections (Figure 1.4A, middle and lower, postnatal day (P) 5-7) into the cochlea via the round window, which had proven highly successful for transduction of hair cells (e.g. (Akil et al., 2012; Jung et al., 2015). We employed AAVPHP.B, a novel AAV-serotype (Deverman et al., 2016) with improved efficiency of neural transduction, for expression of Chronos-ES/TS and Chronos (hSyn promoter, comparable titers, $10^{12} \mathrm{GC} \mathrm{ml}^{-1}$ ) in SGNs. Twelve out 12 AAV-PHP.B-injected mice showed substantial SGN transduction in immunohistochemistry for Chronos-ES/TS and 9 out 9 for Chronos. Postnatal injection of AAV-PHP.B drove strong expression of Chronos-ES/TS across all cochlear turns (Figure 1.4C). Similar to HEK-293T cells and hippocampal neurons in vitro, Chronos-ES/TS localized preferentially peripheral, likely plasmalemmal in SGNs in vivo, while the original Chronos construct was more diffusely distributed throughout the cell (Figure 1.4D). Once again, we quantified the cellular distribution of the opsin by line profile analysis (Figure 1.4E) and found a significantly greater peripheral than central immunofluorescence for Chronos-ES/TS when compared to the original Chronos construct (Figure 1.4E, $p=4.1 \mathrm{e}-5$ ). The difference can be appreciated by the $0.56 \mu \mathrm{m}$ more intracellular $50 \%$ FP-immunofluorescence for Chronos. We note that the cytosolic parvalbumin immunofluorescence allowed a better estimation of the cell border which was independent of 
the opsin expression, both advantageous when compared to HEK-293T cell analysis (Figure 1.2). Despite some differences in absolute numbers between both analyses, which are not unexpected given the different cell types, means of transfection and analysis method, both support the main observation: improved relative plasma membrane abundance of ChronosES/TS.

A

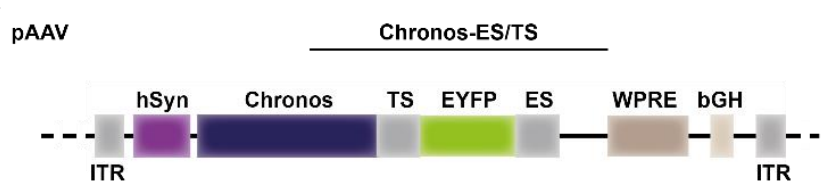

B
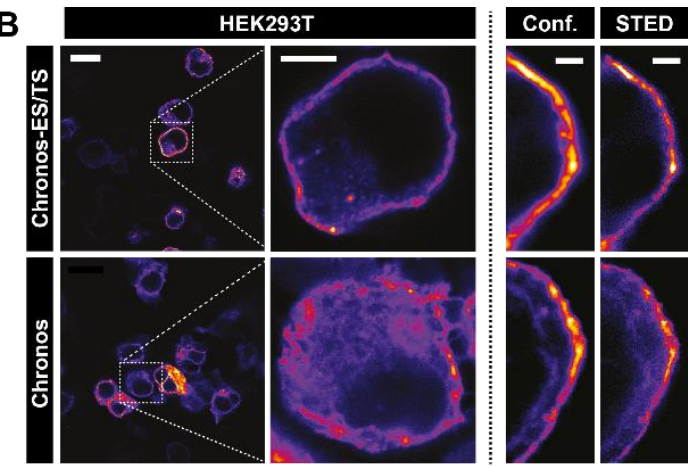

C
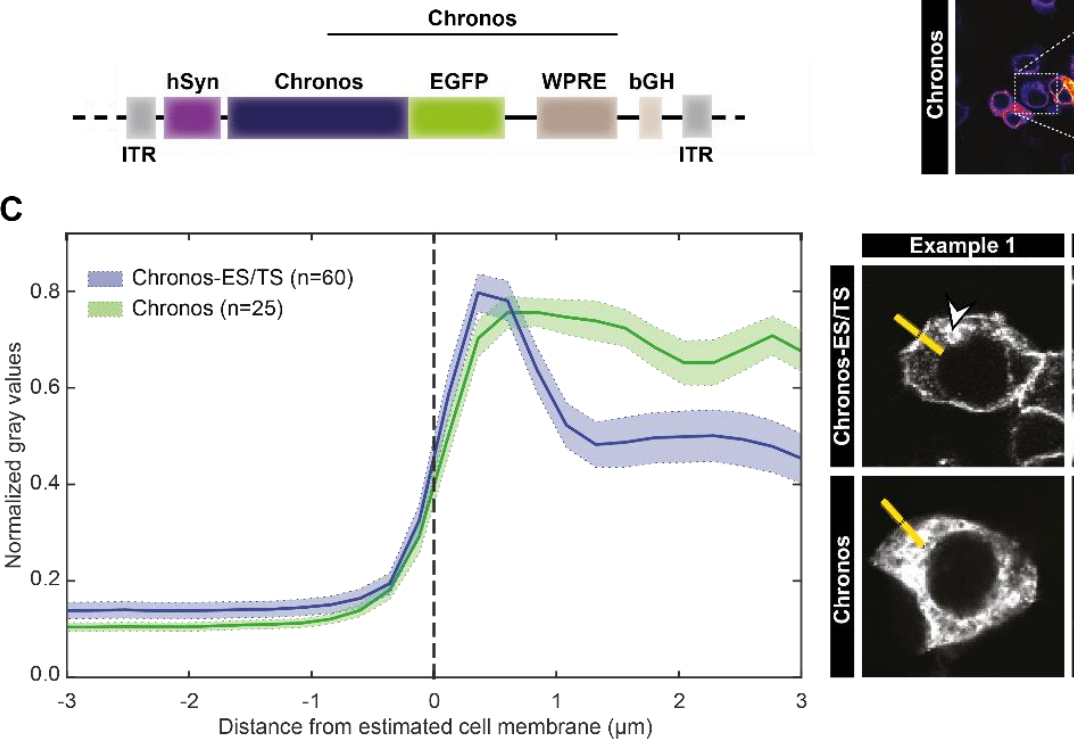

D

Figure 1.2: Optimizing membrane expression of Chronos by adding ER-exit and trafficking signals: HEK-293T cells

A: pAAV vector used in the study Chronos with a trafficking signal (TS), EYFP and ER export signal (ES) Chronos-ES/TS (upper) or containing the original Chronos-EGFP (Klapoetke et al., 2014), lower). In each, expression was driven by the human synapsin promoter (hSyn) and enhanced by the Woodchuck hepatitis virus posttranslational regulatory element (WPRE) and bovine Growth Hormone (bGH) polyadenylation signal (bGH poly A) sequences. ITR: inverted terminal repeats. B: Confocal and STED section of representative HEK-293T cells transfected with ChronosES/TS (upper) and Chronos (lower) and immunolabeled for FP: membranous labeling is more obvious for Chronos-ES/TS. Scale bars: 10,5 and $2 \mu \mathrm{m}$ for left, middle and right panels. C: Peak-normalized line profiles $(7.5 \mu \mathrm{m})$ centered on the estimated membrane of HEK-293T cells expressing Chronos-ES/TS (blue) or Chronos (green) as in B: mean \pm s.e.m. Chronos-ES/TS-expressing cells showed a clear peripheral, likely membrane peak, which is missing in Chronos-expressing cells. Inset shows box and whisker plot of the maximum peak at membrane. Right panels show exemplary line profile placements. One line per cell was placed perpendicular and centered to cell edge, aiming at sufficient intracellular coverage and avoiding fluorescent aggregates (arrowheads). $N$ corresponds to analyzed cells (1 sample/cell). D: Box and whisker plot of the ratio of membrane and cytoplasmic fluorescence of immunolabeled HEK-293T cells expressing Chronos-ES/TS or Chronos: Mann-Whitney U test showed significantly higher ratio in Chronos-ES/TS cells demonstrating an improved membrane expression of Chronos-ES/TS 
$(p=4.4 \mathrm{e}-4)$. The horizontal line within the box indicates the median, boundaries of the box indicate the 0.25 - and 0.75 -percentile, and the whiskers indicate the highest and lowest values of the results. Squares: individual data points. For details on membranous and cytoplasmic area, see Materials and Methods section.
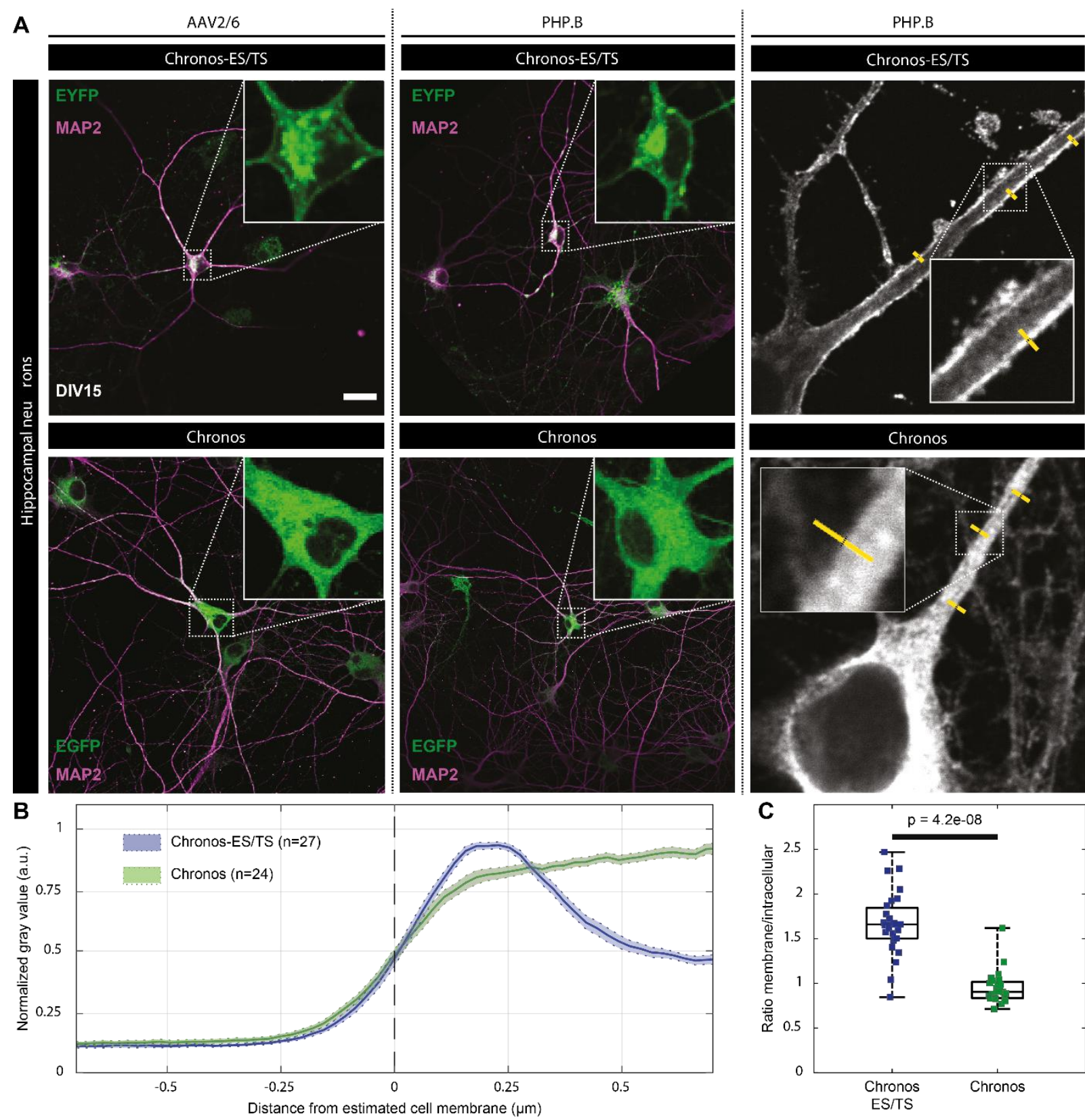

Figure 1.3: Improved membrane expression of optimized Chronos-ES/TS in hippocampal neurons

A: Hippocampal neurons infected at DIV 10 with two different versions of Chronos show very distinct expression patterns. Neurons infected with Chronos-ES/TS (upper panels) either using AAV2/6 (left) or PHP.B virus (middle) show very specific plasma membrane expression in somatic regions and proximal dendrites. Infection of neurons with Chronos (lower panels) either using AAV2/6 (left) or AAV-PHP.B (middle) showed more intracellular opsin abundance. Right panels show exemplary line profile placement (yellow). Scale bar: $50 \mu \mathrm{m}$ applies to all panels. B: Peak-normalized line profiles $(1.5 \mu \mathrm{m})$ centered on the outer cell edge of AAV-PHP.B transduced hippocampal proximal dendrites expressing ChronosES/TS (blue) or Chronos (green) as in A: mean \pm s.e.m. Chronos-ES/TS-expressing cells showed a clear peripheral, likely plasmalemmal peak, which is missing in Chronos-expressing 
cells. C: Box and whisker plot of the ratio of membrane and intracellular fluorescence of immunolabeled hippocampal neurons expressing Chronos-ES/TS or Chronos: Mann-Whitney U-test showed significantly higher ratio in Chronos-ES/TS cells demonstrating an improved membrane expression of Chronos-ES/TS $(p=4.2 \mathrm{e}-8)$. The horizontal line within the box indicates the median, boundaries of the box indicate the 0.25 - and 0.75 -percentile, and the whiskers indicate the highest and lowest values of the results. Squares: individual data points. For details on membranous and cytoplasmic area, see Materials and Methods section.

When analyzing the transduction rates in the injected (left) and non-injected (right) cochleae, we confirmed robust SGN transduction across all turns of the injected cochlea for both Chronos-ES/TS and Chronos (Figure 1.4F). We note that the counterstain for parvalbumin- $\alpha$, used for AAV-PHP.B injected ears, is a more general marker of SGNs than calretinin, used for AAV2/6 injected ears, which is present only in a subset of SGNs. Therefore, if anything, we would have been prone to overestimate the transduction rate for AAV2/6 injected ears, which, however, was very low. Interestingly, for both cases of AAV-PHP.B injection we also found substantial expression in the contralateral, non-injected cochleae, indicating spread of virus in the specific conditions of pressure injection into the scala tympani of the early postnatal cochlea. This spread likely occurred via the cochlear aqueduct and/or the endolymphatic ducts and the cerebrospinal fluid space (Lalwani et al., 1996). The density of SGNs in the injected as well as in non-injected ears were comparable (Figure 1.4G). The injected mice behaved normal as concluded from routine animal observation. 
A
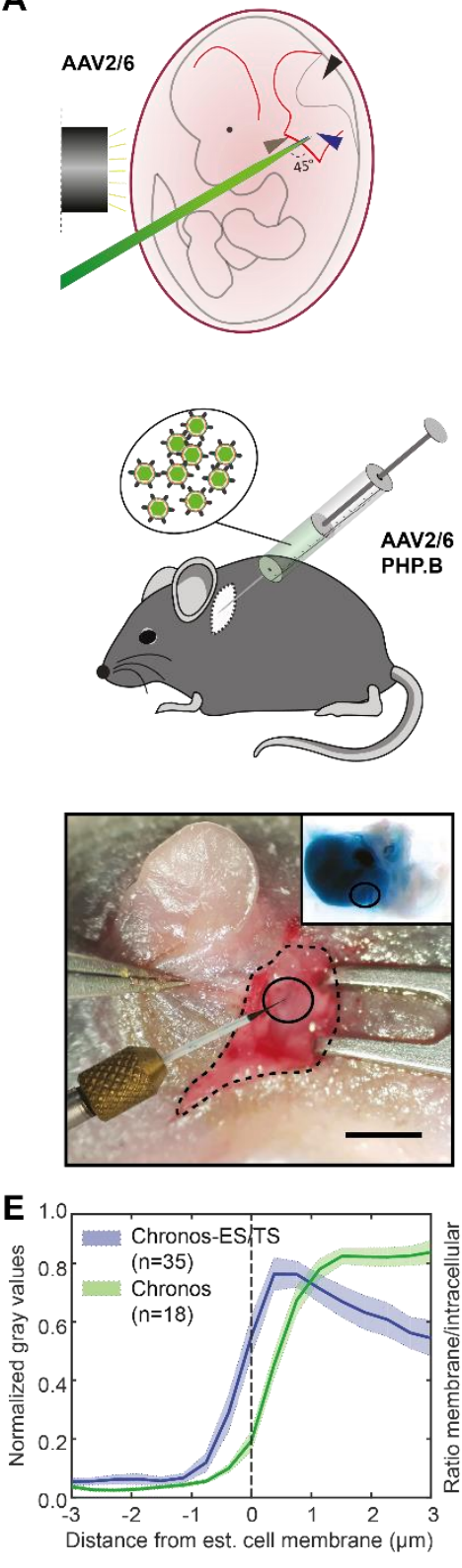

B

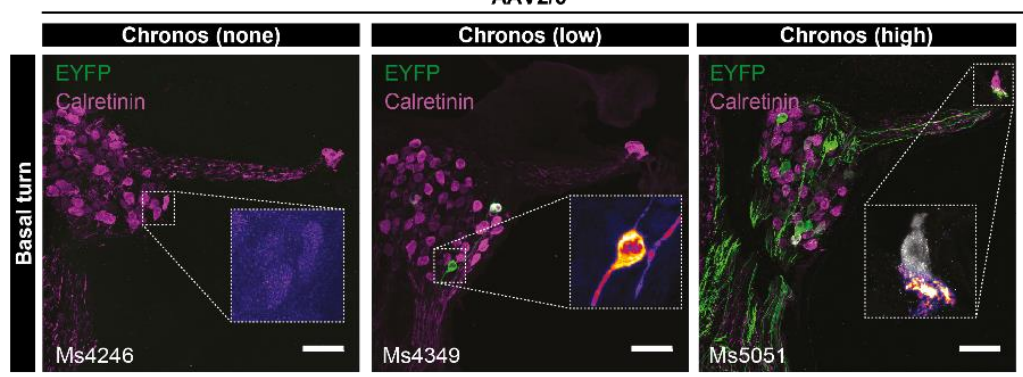

C

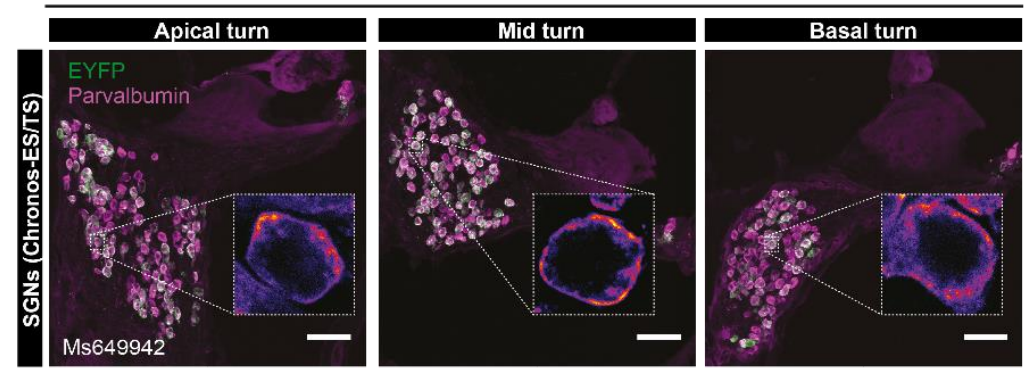

D
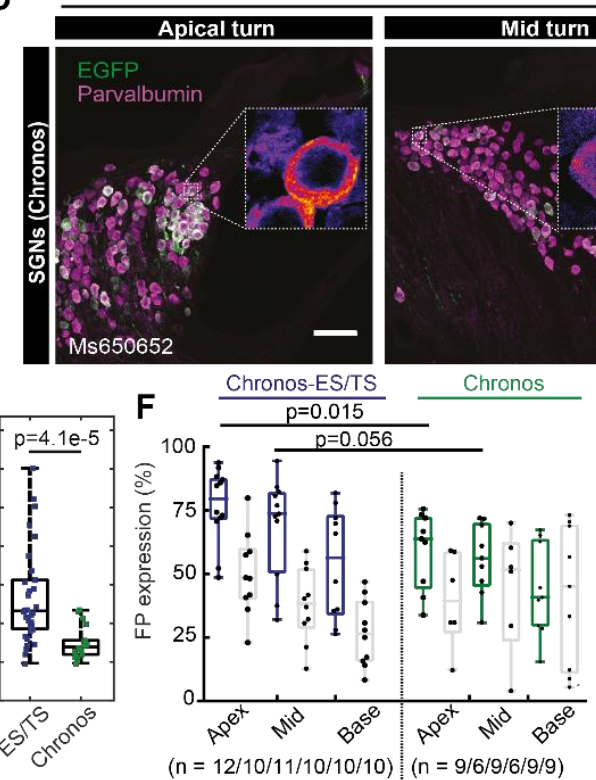

PHP.B
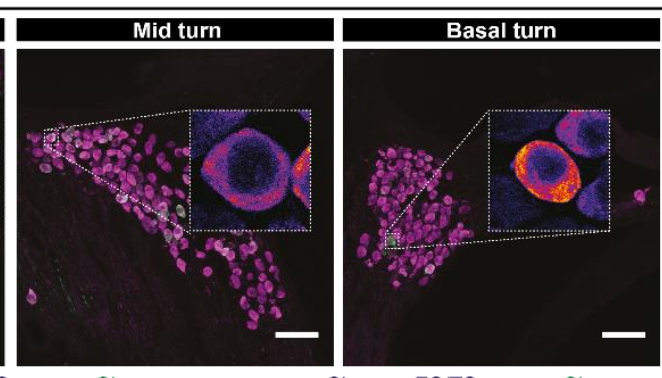

G

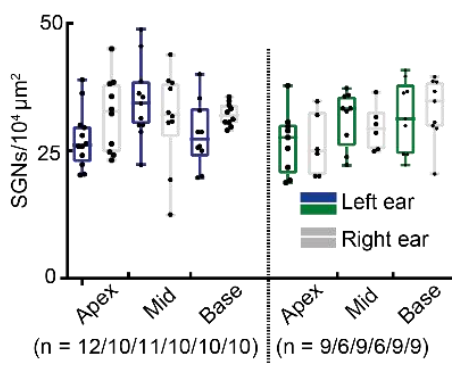

Figure 1.4: Establishing efficient expression of Chronos in SGNs: use of Chronos-ES/TS, potent AAV-PHP.B, and postnatal mode of AAV-injection

A: Upper panel: schematic representation of the viral injection into the embryonic otocyst (left: black cylinder marks the light guide used to trans-illuminate the embryo in the uterus after mobilization from the abdominal cavity, green: micropipette filled with fast greencolored AAV suspension). Middle panel: schematic representation of AAV-injection into the postnatal cochlea via the round window (RW). Lower panel: surgical situs of a P7 mouse with retroauricular incision, graphical aid encircles the injection site). Inset shows ex-vivo cochlea just after AAV injection via RW. Scale bar: $2 \mathrm{~mm}$. B: Maximum projection of confocal images of immunolabeled mid-modiolar cochlear cryosections (exemplary sections of basal turn) of embryonically AAV2/6-Chronos-injected mice collected at 4-weeks of age. EYFP (green) marks transduced SGNs, calretinin (magenta) was used as generic marker of SGNs, scale bar: $50 \mu \mathrm{m}$. In the inset, color code for EYFP channel was changed to fire (EYFP). Left panel: most common, non-expressing example, inset shows zoom of negative SGNs. Middle panel: occasional, sparsely expressing example, inset: one out of two positive SGNs. Right panel: rare, highly expressing example, inset: negative inner hair cell (calretinin in gray), exclusive localization of EYFP in the SGN boutons and fibers. C: Postnatally AAV-PHP.B- 
Chronos-ES/TS-injected mouse (see B except where stated differently). EYFP (green) marks transduced SGNs, parvalbumin (magenta) was used as generic marker of SGNs, scale bar: $50 \mu \mathrm{m}$. High transduction rate, good membrane expression. In inset, color code for the green channel was changed to fire for better visualization. Similar to B. D: Postnatally AAVPHP.B-Chronos-injected mouse (see C except where stated differently). Substantial SGN transduction, poor membrane expression. E: Line profile analysis of FP-immunofluorescence across the membrane of SGN somata. Traces were centered at the transition from high to low parvalbumin immunofluorescence as a proxy of plasma membrane location. Left panel: clear membrane abundance in Chronos-ES/TS (blue, mean \pm s.e.m.), but mostly intracellular localization in Chronos (green, mean \pm s.e.m.). Inset shows box and whisker plot of the maximum fluorescence at the membrane. Right panel: Box and whisker plot of the ratio of membrane and intracellular FP fluorescence for Chronos-ES/TS (left) and Chronos (right): stronger relative membrane expression in Chronos-ES/TS (Mann-Whitney U test, $p=4.1 \mathrm{e}-5$ ). Squares: individual data points. For details on membranous and cytoplasmic area, see Materials and Methods section. F: Box and whisker plot of the fraction of FP-positive SGNs (transduced out of all parvalbumin-positive SGNs) for the apical, middle and basal cochlear turn of the injected ear (colored) and contralateral, non-injected (grey) ear of Chronos-ES/TSAAV-PHP.B and Chronos-AAV-PHP.B mice. Points: individual animals plotted on top; $n$ refers to number of cochleae studied. The horizontal line within the box indicates the median, boundaries of the box indicate the 0.25- and 0.75-percentile, and the whiskers indicate the highest and lowest values of the results. G: Box and whisker of the SGN density for the apical, middle and basal cochlear turn of the injected (colored) ear and the contralateral, noninjected (grey) ear (as in F). No significant differences between Chronos and Chronos-ES/TS nor between injected and non-injected ear. Points: individual cells plotted on top, $n$ as stated in $\mathrm{F}$.

Chronos-mediated optogenetic neural stimulation of the auditory pathway: transuterine injections of $A A V 2 / 6$

We performed acute recordings of oABRs using fiber-coupled laser stimulation 4-12 weeks after AAV-injection. Following posterior tympanotomy, we inserted a $50 \mu \mathrm{m}$ optical fiber through the round window (RW) to project the light of a blue laser $(\lambda=473 \mathrm{~nm})$ onto the SGNs. When oABRs were present (Figure EV1.1), we typically found three to five oABR peaks, which likely reflected the synchronous activation of Chronos-expressing SGNs (first peak) and downstream auditory pathway (subsequent peaks). Transuterine injections of AAV2/6-Chronos did not generally support oABRs; only 3 out of dozens of injected mice (including 2 different AAV2/6 produces) showed oABRs. In these positive mice, oABR grew in amplitude and showed shorter latencies when increasing radiant flux (see inset of Figure EV1.1).

\section{Chronos-ES/TS enables ultrafast optogenetic stimulation of the auditory pathway: oABRs}

Postnatal injection of AAV-PHP.B reliably achieved high transduction rates (Figure 1.4), which enabled oABRs in 19 out of 20 AAV-PHP.B-Chronos-ES/TS-injected mice and 8 out of 17 AAV-PHP.B-Chronos-injected mice. Next, we performed a detailed characterization of Chronos-ES/TS-mediated optogenetic activation using oABRs and compared the results to 
those obtained with Chronos. oABR amplitude grew with increasing stimulus intensity (Figure 1.5A,B, $1 \mathrm{~ms}$ light pulses delivered at $10 \mathrm{~Hz}$ - averaged across 1000 repetitions), while oABR latency (i.e. the time interval between the stimulus onset and the oABR $\mathrm{P}_{1}$ wave) got shorter for Chronos-ES/TS (sign test between the threshold and highest radiant flux tested, $p=0.009$ ), which did not reach statistical significance for Chronos (Figure 1.5A,C; $p=0.11$ ). oABR thresholds and amplitudes differed between animals (shown for the first peak, $\mathrm{P}_{1}-\mathrm{N}_{1}$, Figure 1.5B). Stimuli as weak as $1.08 \mathrm{~mW}$ (duration: $1 \mathrm{~ms}$, rate: $10 \mathrm{~Hz}$ - averaged across 1000 repetitions) were sufficient to drive oABRs in one Chronos ES/TS injected mouse and $4.56 \mathrm{~mW}$ in a Chronos injected mouse. For $1 \mathrm{~ms}$ light pulses delivered at $10 \mathrm{~Hz}$, the average oABR threshold amounted to $6.58 \pm 1.08 \mathrm{~mW}$ and $13.95 \pm 3.52 \mathrm{~mW}$ for Chronos-ES/TS and Chronos, respectively ( $p=0.0367$, Mann-Whitney $\mathrm{U}$ test; $n=13$ and $n=8$ ). In most animals oABR amplitudes grew with radiant flux increasing over more than one order of magnitude (Figure 1.5B). The minimal latency of the first oABR peak $\left(\mathrm{P}_{1}\right.$, Figure $\left.1.5 \mathrm{C}\right)$ amounted to $0.95 \pm 0.07 \mathrm{~ms}(\mathrm{n}=13)$ for Chronos-ES/TS and $1.26 \pm 0.07 \mathrm{~ms}$ for Chronos $(\mathrm{n}=8, p=0.014$, Mann-Whitney U test).

oABRs could be elicited by light pulses as short as $20 \mu \mathrm{s}$ with Chronos-ES/TS and $400 \mu \mathrm{s}$ with Chronos (irradiance: $38 \mathrm{~mW}$, rate: $10 \mathrm{~Hz}$ - averaged across 1000 repetitions, Figure 1.5D,E). oABR amplitudes grew with pulse duration up to approximately $0.6 \mathrm{~ms}$ and tended to become smaller for longer pulses, possibly due to accumulating channel inactivation and/or increasing depolarization-block of SGNs upon prolonged photo-depolarization (Figure 1.5D,E). 


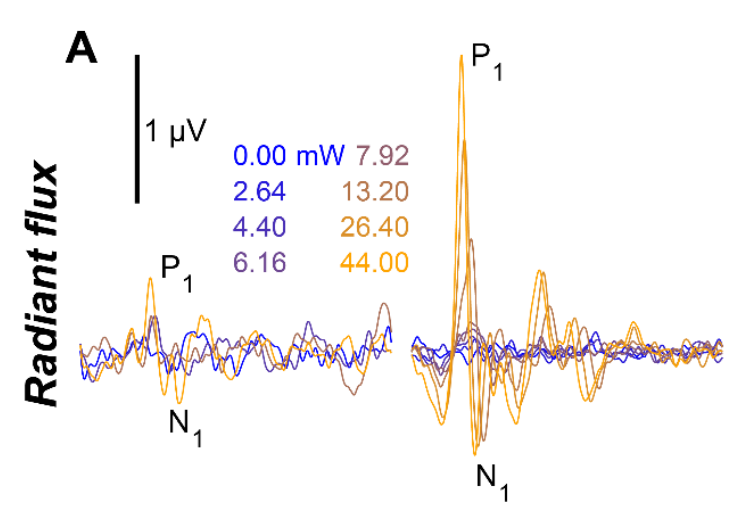

Chronos Chronos-ES/TS
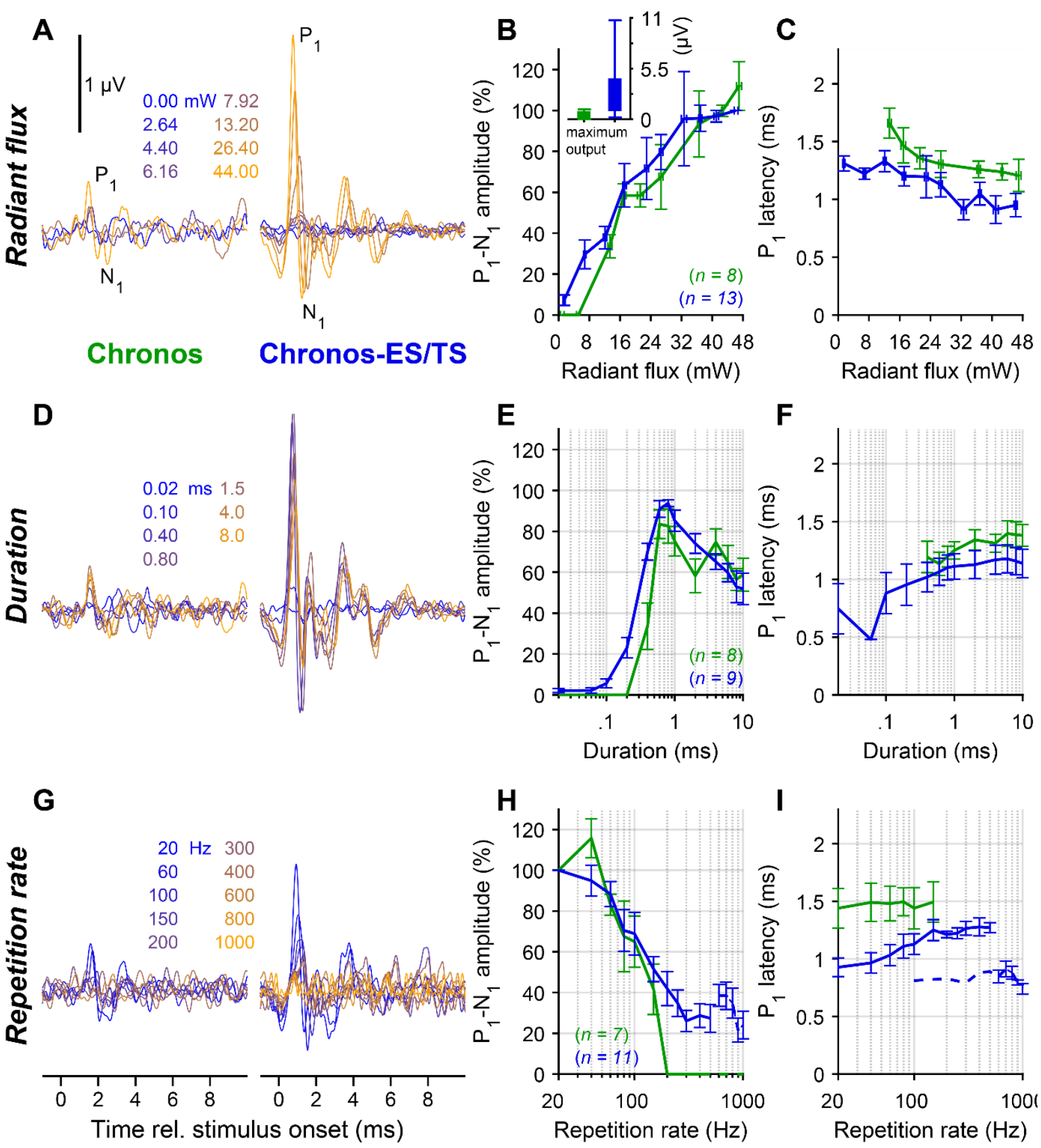

Figure 1.5: Characterizing optogenetic stimulation by recordings of oABRs

A: oABRs driven with varying radiant flux $(1 \mathrm{~ms}$ at $10 \mathrm{~Hz}$, colors code the radiant flux in $\mathrm{mW}$ ) for exemplary mice injected with AAV-PHP.B-Chronos (left) or AAV-PHP.B-ChronosES/TS (right). B: Normalized $\mathrm{P}_{1}-\mathrm{N}_{1}$ amplitude of oABR as a function of light intensity for Chronos as in A (green, throughout figure) and Chronos-ES/TS (blue). Radiant flux was binned per $5 \mathrm{~mW}$ steps (horizontal error: \pm s.e.m.). Inset: maximal absolute $\mathrm{P}_{1}-\mathrm{N} 1$ amplitude of oABR (mean \pm s.e.m.) was greater for Chronos-ES/TS than for Chronos $(p$ - $\leq 0.001$; Mann-Whitney-U test). C: Latency of oABR $\mathrm{P}_{1}$ as a function of radiant flux as in B. Radiant flux was binned per $5 \mathrm{~mW}$ steps (horizontal error: \pm s.e.m.). D: oABRs driven with varying stimulus duration $(10 \mathrm{~Hz}, 38 \mathrm{~mW}$, colors code the duration) for exemplary mice injected with AAV-PHP.B-Chronos (left) or AAV-PHP.B-Chronos-ES/TS (right). E: $\mathrm{P}_{1}-\mathrm{N}_{1}$ amplitude as a function of stimulus duration as in $\mathrm{D}$ (normalized against $\mathrm{P}_{1}-\mathrm{N} 1$ oABR amplitude. F: Latency of $\mathrm{oABR} \mathrm{P}_{1}$ as a function of stimulus duration as in $\mathrm{E}$. G: oABRs driven with varying stimulus rate $(1 \mathrm{~ms}$ and $0.5 \mathrm{~ms}$ for $10-500 \mathrm{~Hz}$ and $500-1000 \mathrm{~Hz}$, respectively, $38 \mathrm{~mW}$, colors code the stimulus rate) for exemplary mice injected with AAV-PHP.B-Chronos (left) or 
AAV-PHP.B-Chronos-ES/TS (right). H: $\mathrm{P}_{1}-\mathrm{N}_{1}$ amplitude as a function of stimulus rate as in $\mathrm{G}$ (normalized against $\mathrm{P}_{1}-\mathrm{N}_{1} \mathrm{oABR}$ amplitude at $20 \mathrm{~Hz}$ ). I: Latency of oABR $\mathrm{P}_{1}$ as a function of stimulus rate as in $\mathrm{H}$ : Chronos-ES/TS enables responses up to at least $1000 \mathrm{~Hz}$. Dashed line indicates latency data points obtained with a pulse duration of $0.5 \mathrm{~ms}$.

Data information: The $\mathrm{P}_{1}-\mathrm{N}_{1}$ amplitude and $\mathrm{P}_{1}$ latency were measured on the same animals. Animal count $(n)$ is stated on bottom right of panel B, E, and H. Data are expressed as mean \pm s.e.m.

Next, we tested the dependence of oABRs on the stimulus rate [duration: $1 \mathrm{~ms}$ up $500 \mathrm{~Hz}$ and $0.5 \mathrm{~ms}$ from $500 \mathrm{~Hz}$, intensity: maximum $(38-43 \mathrm{~mW})]$. When increasing stimulus rate, oABR amplitudes declined (Figure 1.5G) and latencies prolonged (Figure 1.5G,I). However, in contrast to our previous reports on: i) $\mathrm{ChR} 2$ : where potentials were found only up to $70 \mathrm{~Hz}$ (Hernandez et al., 2014a); ii) CatCh: up to $200 \mathrm{~Hz}$, and f-Chrimson: up to $250 \mathrm{~Hz}$ (respectively (Wrobel et al., 2018; Mager et al., 2018), we could detect sizable $\mathrm{P}_{1}-\mathrm{N}_{1}$ up to stimulus rates of $500 \mathrm{~Hz}$ for Chronos (Figure 1.5G,H) and $1000 \mathrm{~Hz}$ for Chronos-ES/TS (the highest tested in our experiments, Figure $1.5 \mathrm{G}-\mathrm{H}) . \mathrm{P}_{1}$-latency increased with higher stimulus rates in both cases.

Chronos-ES/TS enables ultrafast optogenetic stimulation of the auditory pathway: recordings from single putative SGNS

To further validate the Chronos-ES/TS-mediated SGN stimulation and evaluate the temporal fidelity of stimulation, we performed juxtacellular recordings from auditory nerve fibers (central axon of SGN) as described in (Hernandez et al., 2014a; Mager et al., 2018). In brief, we targeted glass micropipettes to where the auditory nerve enters the anteroventral cochlear nucleus (AVCN) and searched for responses while stimulating the SGNs through the round window via an optical fiber coupled to a blue laser. We favored recordings of SGNs by deep positioning ( $>1000 \mu \mathrm{m}$ relative to the surface of the cochlear nucleus) of the pipette tip, but, given that responses to acoustic stimuli were lost upon the ear surgery, we could not safely discriminate SGNs from AVCN neurons, hence, we termed the light-responsive neurons "putative SGN". We found that the putative SGNs fired upon optogenetic stimulation with high temporal precision for stimulus rates of up to hundreds of $\mathrm{Hz}$ (Figure 1.6A, B); some neurons followed stimulation to some extent even up to $1000 \mathrm{~Hz}$ (Figure 1.6B). Temporal precision of firing, evaluated based on vector strength (Goldberg and Brown, 1969), see Materials and Methods, Figure 1.6C) and spike jitter (i.e., standard deviation of spike latency across trials, Figure 1.6D), was generally high, but varied across the recorded neurons.

Temporal precision and spike probability (Figure 1.6E) diminished with increasing stimulation rates, indicating that single SGNs code optogenetic information in a less reliable 
manner at very high stimulation rates. Spike jitter, calculated for spikes occurring in a time window comprised between two pulse onsets, increased with rate but it was typically below a millisecond for stimulus rates lower than $300 \mathrm{~Hz}$ (Figure 1.6D). At higher stimulus rates, spike jitter increased beyond the values obtained for simulated Poisson spike trains (see Materials and Methods, red-shaded area, Figure 1.6D), indicating that spike synchronization with the light pulses became less reliable. The lower spike precision and limited spike probability at stimulus rates beyond $100 \mathrm{~Hz}$ observed are likely compensated by the population response, as several SGNs jointly encode information from each place of the tonotopic map (Liberman, 1978).

Light-evoked firing rate patterns differed among the putative SGNs as stimulation rates increased (Figure 1.6F). There was, in general, a linear rise of discharge rates up to $100 \mathrm{~Hz}$, followed by a constant decrease as a response to mid and high stimulation rates. Nevertheless, some putative SGNs were able to maintain moderate (and to some extent synchronized) spike rates even at pulse rates of $1000 \mathrm{~Hz}$ (fibers showing computable spike probability — see Materials and Methods- at $500 \mathrm{~Hz}: 7 / 21,33.3 \%$; at $600 \mathrm{~Hz}: 3 / 18,16.7 \%$; at $700 \mathrm{~Hz}: 3 / 18$, 16.7\%; at $800 \mathrm{~Hz}: 1 / 19,5.26 \%$; at $900 \mathrm{~Hz}: 2 / 21,9.52 \%$; and at $1000 \mathrm{~Hz} 2 / 19,10.53 \%$ ). Interestingly, the response patterns of the putative SGNs that we recorded varied across units at increasing stimulation rates (Figure EV1.2). We found two main types of responses; putative SGNs that rapidly adapted their spiking as stimulation rates rose (Figure EV1.2A), and neurons that continued spiking even at very high pulse rates (albeit with a lower frequency as compared to lower stimulation rates, Figure EV1.2B). 


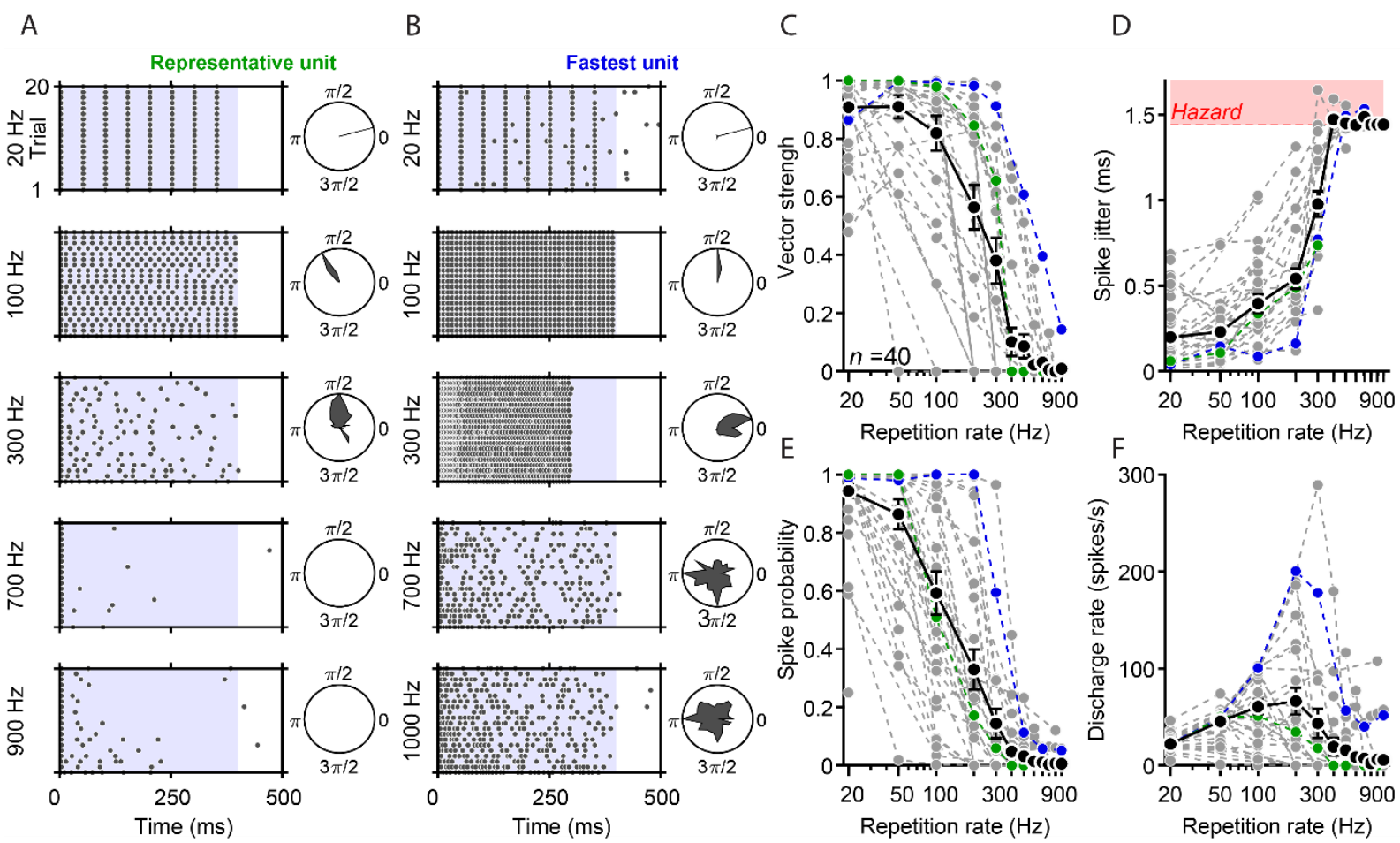

Figure 1.6: Characterizing optogenetic stimulation by juxtacellular recordings from single putative SGNs

A: Raster plots showing spiking activity from a representative putative SGN ("unit") in response to $400 \mathrm{~ms}-$ long trains of laser pulses (at $30 \mathrm{~mW}, 1 \mathrm{~ms}$ for $20-600 \mathrm{~Hz}, 500 \mu \mathrm{s}$ for $\geq 700 \mathrm{~Hz}$ ) recorded at five different stimulation rates over 20 repetitions. Polar plots to the right side of raster plots show synchronicity of firing relative to the cycle between two pulse onsets. B: Same protocol and analysis of the "fastest unit" recorded, showing lower adaptation and firing even at very high stimulation rates. C-F: Quantification of the vector strength $(\mathbf{C})$, spike jitter $(\mathbf{D})$, spike probability $(\mathbf{E})$ and discharge rate $(\mathbf{F})$ as a function of repetition rate of 40 putative SGNs (obtained from 6 mice). In panel D, the red-shaded area represents the hazard function obtained in response to simulated Poisson spike trains.

Data information: Black data points and lines show mean \pm s.e.m.; individual units are shown in gray. The units shown on A-B are shown in green and blue, respectively. Number of units tested for each repetition rate: $20 \mathrm{~Hz}: 40,50 \mathrm{~Hz}: 25,100 \mathrm{~Hz}: 24,200 \mathrm{~Hz}: 26,300 \mathrm{~Hz}: 21,400$ Hz: 20, 500 Hz: 21, 600 Hz: 18, 700 Hz: 18, 800 Hz: 19, 900 Hz: 21, 1000 Hz: 19.

\section{Discussion}

Here, we characterized and optimized Chronos for its utility in fast optogenetic control of excitable cells. We demonstrate sub-millisecond off-kinetics at physiological temperature of Chronos. Using the auditory system as a fast spiking neural model circuitry, we show that Chronos supports ultrafast control of neuronal spiking. In doing so, we solved a major shortcoming that plagued optogenetics as a stimulus modality for the cochlea; the low temporal fidelity of light-driven SGN-firing, caused by the slow deactivation kinetics of the ChR2 employed so far. We found that adding sequences promoting ER-exit and plasma membrane trafficking to Chronos (Chronos-ES/TS) and using postnatal injection of the powerful AAV-PHP.B vector critically improved the in vivo utility. In conclusion, Chronos- 
ES/TS in combination with potent viral vectors such as AAV-PHP.B is a promising tool for auditory neuroscience and a candidate ChR for use in future optical CIs.

\section{Biophysical characterization of Chronos and improving plasma membrane expression}

Here, we compared activation and deactivation for ChR2 and Chronos and also studied the temperature dependence of gating for Chronos. In our hands Chronos deactivates about 3 times faster than ChR2 and has sub-milliseconds off-kinetics at physiological temperature with a $\mathrm{Q}_{10}$ of 2.7. This reflects short-lived open states for Chronos resulting in lower open probability, which together with the relatively poor membrane expression likely explains the challenges we faced with using the original Chronos construct for driving SGN spiking, when using the transuterine injection in the AAV2/6-hSyn vector that we previously used successfully for the ChR2 mutant CatCh (Hernandez et al., 2014a). No problems were reported in two other studies of Chronos in the auditory system using different viruses and stages of the pathway; cochlear nucleus (Hight et al., 2015) and inferior colliculus (Guo et al., 2015). A recent study using the in silico predicted ancient AAV Anc80 also achieve functional expression in the cochlea, but did not differentiate between expression in SGNs and hair cells (Duarte et al., 2018). Inspecting the Chronos-GFP expression in the cochlear nucleus (Figure 2 of (Hight et al., 2015)) and SGNs (Duarte et al., 2018) suggest a similar diffuse intracellular distribution as we observed in HEK-293T cells (Figure 1.2) and hippocampal neurons (Figure 1.3) in culture as well as in SGNs in our study (Figure 1.4). Using these 3 cell types and vastly different conditions, we show a robust improvement of the plasma membrane expression of Chronos upon adding ER-export (Ma et al., 2001; Stockklausner et al., 2001) and trafficking signals (Hofherr et al., 2005) of inward rectifying $\mathrm{K}^{+}$channels. Introduction of the powerful AAV-variant AAV-PHP.B (Deverman et al., 2016) was an additional factor changing the game: even the unmodified Chronos (Klapoetke et al., 2014) enabled oABRs on a regular basis. However, when comparing the functionality of Chronos-ES/TS with enhanced membrane trafficking to Chronos, transduced by the same AAV-PHP.B vector, titer, injection method and incubation time, we found increased oABR amplitudes and improved temporal fidelity of the Chronos-ES/TS-mediated responses, when increasing the rate of stimulation. Disentangling the contributions of the improved membrane expression and the slightly higher fraction of Chronos-positive SGNs with Chronos-ES/TS is challenging. Regardless of the precise contributions, these experiments suggest that ChronosES/TS will be a valuable tool for optogenetic applications requiring good temporal fidelity. Moreover, avoiding the largely intracellular localization of Chronos lowers the proteostatic stress to the cell. 


\section{Ultrafast Chronos-mediated stimulation of the auditory pathway}

Stimulation of the auditory pathway is a prime example for an application of optogenetics requiring both high speed and light sensitivity. Upon sound stimulation, SGNs fire at hundreds of $\mathrm{Hz}$ and show sub-millisecond temporal precision of spiking relative to the stimulus. When considering optogenetics for improved cochlear prosthetics, each stimulus should not exceed the energy requirements of electrical cochlear implants which are in the range of $0.2 \mu \mathrm{J}$ per pulse (Zierhofer et al., 1995). However, so far, the threshold for optogenetic activation of SGNs reported for $\mathrm{ChR} 2$ and $\mathrm{ChR} 2$ variant $\mathrm{CatCh}$ amounted to approximately $2 \mu \mathrm{J}$, and the responses broke down for stimulation rates exceeding $20 \mathrm{~Hz}$ (Hernandez et al., 2014a).

Chronos, featuring a sub-millisecond deactivation time constant at physiological temperature (Figure 1.1) is an obvious candidate for cochlear optogenetics. So far, we had employed transuterine AAV2/6-injections into the embryonic otocyst and observed a strong basoapical gradient of expression in the cochlea (Hernandez et al., 2014a). Using AAV-PHP.B, a novel AAV vector (Deverman et al., 2016) at high titers (1-2.5 $\left.10^{12} \mathrm{GC} / \mathrm{ml}\right)$ for injections into the cochlea of P5-P7 mice, we found highly efficient transduction of SGNs across all cochlear turns (Figure 1.5). The light thresholds estimated for oABRs mediated by postnatal AAVPHP.B injections of Chronos-ES/TS in SGNs amounted to $7 \mu \mathrm{J}$ (14 $\mu \mathrm{J}$ for Chronos), which is substantially higher than the energy per pulse for suprathreshold stumulation in eCIs. Therefore, further improvements of membrane expression of Chronos or the design of fast channels with larger pore remain an important objective. At present, we can only speculate how much light will be required for an auditory percept mediated by Chronos-based cochlear optogenetics. Future studies using behavioral experiments will be required to address this point. Nonetheless, we argue that future optogenetic CIs might not need the high stimulation rates employed in eCIs ( $800 \mathrm{~Hz}$ or higher) which might help balancing a greater energy requirement per pulse of an oCI. In most animals, oABR increased in amplitude when increasing light intensity over more than one order of magnitude (Figure 1.6). Hence, the output dynamic range of optical stimulation assessed as $\mathrm{P}_{1}-\mathrm{N}_{1}$ amplitude, on average, was $>10 \mathrm{~dB}(\mathrm{~mW})$ compared to typically $<10 \mathrm{~dB}$ for coding with eCI (Zeng et al., 2008). This likely reflects the lower spread of excitation with optical stimulation as well as differences in the levels of Chronos-ES/TS expression among SGNs at the same tonotopic place of stimulation. 
The temporal fidelity of Chronos-ES/TS-mediated optogenetic stimulation was estimated at the single SGN and the SGN population levels. The minimal oABR latency was considerably shorter $(1.01 \pm 0.09 \mathrm{~ms})$ than that of the first light-evoked potential, typically a trough, that we had previously reported for transgenic ChR2 mice $(3.14 \pm 0.26 \mathrm{~ms}$ (Hernandez et al., 2014a)). Moreover, the minimal duration of the light pulse required for eliciting an oABR at high light intensity was shorter $(<100 \mu \mathrm{s})$ than that found before with $\mathrm{ChR} 2$ or CatCh ( $\geq 200 \mu$ s; (Hernandez et al., 2014a)). Most importantly, population responses mediated by Chronos-ES/TS followed pulse rates up to at least $1000 \mathrm{~Hz}$ unlike for ChR2, where oABRs were lost below $100 \mathrm{~Hz}$. Obviously, the analysis had to be restricted to a very short measurement time window in order to track the response to such high rates and hence signal propagation along the pathway cannot be demonstrated. This ABR analysis suggests that the Chronos-ES/TS mediated optogenetic stimulation achieves a temporal fidelity similar to that of acoustic coding. Using juxtacellular recordings we could demonstrate firing of single SGNs in response to trains of light pulses at hundreds of $\mathrm{Hz}$ with sub-millisecond temporal precision. Such temporal fidelity of optogenetic control of SGN firing marks a major breakthrough on the way towards using optogenetic stimulation for auditory research and for the future development of the clinical optical cochlear implant.

\section{Materials and Methods}

\section{Cloning}

For the cloning of pAAV_hSyn_Chronos-ES/TS as a starting material we have used pAAVEf1a-DIO-eNpHR 3.0-EYFP (Addgene, plasmid nr. \#26966). In order to obtain flanked EYFP with ES/TS sequences we performed a classical PCR reaction. The primers that we used for this cloning were 5'-GAGAACCGGTCAAGAGCAGGATCAC-3' and 5'GTGGGGTACCCCTTACACCTCGTTCTC-3'. In the second step, the obtained PCR fragment was digested with AgeI/Acc65I (Thermo Scientific, MA, USA) (Acc65I produces compatible cohesive ends as BsrGI) gel extracted (GeneJET Gel Extraction Kit, Thermo Scientific, MA, USA) and further used for ligation. At the same time, the plasmid pAAV_hSyn_Chronos-GFP (Addgene, plasmid no. 59170) was also digested using restriction enzymes AgeI/BsrGI and used as a backbone plasmid. All obtained ligation products were further tested by the means of colony PCR and finally sequenced by an external company.

\section{Cell Culture and HEK-293T transfection}

HEK-293T cells (ATCC, USA) were cultured at $37^{\circ} \mathrm{C}$ and $5 \% \mathrm{CO}_{2}$ in DMEM (Gibco, USA) supplemented with $10 \%$ fetal calf serum (Gibco, Germany), and $1 \%$ penicillin/streptomycin 
(Sigma, Germany). One day prior to transient transfections the HEK293T cells were seeded on 24-well plates. The day after seeding, cells were transiently transfected with pAAV_hSyn_Chronos-ES/TS or pAAV_hSyn_Chronos using PEI 25.000MW (Polysciences Inc. USA). Two days after transfection cells were briefly washed and then fixed with $4 \%$ PFA for $10 \mathrm{~min}$. After fixation cells were mounted with Mowiol (Sigma, Germany) and processed to confocal/STED imaging. Cells were regularly tested for mycoplasma contamination. No method of cell line authentication was used.

For characterization of gating kinetics, HEK-293T cells were transfected with 3-5 $\mu \mathrm{g}$ of plasmid DNA encoding a ChR2-YFP or a Chronos-GFP fusion protein via nucleofection using a Lonza nucleofector device and the Amaxa Cell Line Nucleofection Kit V (Lonza, Switzerland; program Q-01), following the manufacturer's instructions. The pcDNA 3.1ChR2-YFP construct was kindly provided by Ernst Bamberg (MPI for Biophysics, Frankfurt, Germany), and Chronos-GFP was kindly provided by Edward Boyden (MIT, Cambridge, MA). After transfection, the cells were plated on poly-L-lysine coated $10 \mathrm{~mm}$ glass cover slips. Electrophysiological characterization was performed 20 to $30 \mathrm{~h}$ after transfection.

\section{Electrophysiology}

Transfection success was accessed via fluorescence in an inverted Axiovert $135 \mathrm{TV}$ fluorescence microscope (ZEISS, Germany), equipped with a 40X/0.65 N.A. Achroplan objective. Only cells isolated from others and exhibiting clear membrane fluorescence signal were recorded. Whole-cell patch-clamp recordings were made in voltage-clamp mode ($60 \mathrm{mV}$ holding potential) using an EPC 10 USB amplifier (HEKA Elektronik, Germany). Current signals were low-pass filtered at $3 \mathrm{kHz}$ and digitized at $20 \mathrm{kHz}$. Patch-pipettes were prepared from PG10165-4 glass capillaries (World Precision Instruments, USA) in a PIP 6 vertical puller (HEKA Elektronik, Germany), and had resistances between 3-5 M $\Omega$ when filled with the following pipette solution (in $\mathrm{mM}$ ): $110 \mathrm{NaCl}, 10 \mathrm{Na}_{4}-\mathrm{EGTA}, 4 \mathrm{MgCl}_{2}$, 10 HEPES, and 10 Glucose (pH 7.4 and osmolarity between 285-290 mOsm). The bath solution contained (in $\mathrm{mM}$ ): $145 \mathrm{NaCl}, 3 \mathrm{KCl}, 1 \mathrm{MgCl}_{2}, 2 \mathrm{CaCl}_{2}, 10 \mathrm{HEPES}$, and 15 Glucose (osmolarity between 310-315 mOsm and pH 7.35). Series resistance (always $<20 \mathrm{M} \Omega$ before compensation) was electronically compensated 60 to $90 \%$. For recordings at physiological temperature, warm solution was perfused by gravitation in the recording chamber via an HPT2 in-line heater (ALA Scientific Instruments, USA) controlled by a TC-10 temperature controller (NPI Electronic, Germany). Temperature at the recording chamber was monitored via a thermistor placed in the chamber and it was maintained at $36 \pm 1{ }^{\circ} \mathrm{C}$. Light-stimulation 
was achieved using a $480 \mathrm{~nm}$ diode (5 W Luxeon rebel color with Lambertian dome; Philips Lumileds) controlled by a custom-built controller. Stimulation protocols consisted of 1 s-long light steps at different intensities $\left(0.08,0.14,0.19,0.23,0.27 \mathrm{~mW} \mathrm{~mm}^{-2}\right)$, with a $11 \mathrm{~s}$-long dark period in between, or a 10 s-long light chirp linearly-increasing from 0.1 to 100 (or 500) $\mathrm{Hz}$ and maximum amplitude of $0.27 \mathrm{~mW} \mathrm{~mm}^{-2}$ (following a $1 \mathrm{~s}$-long pre-pulse at half maximum intensity). The LED output faithfully followed the voltage command up to at least $5 \mathrm{kHz}$. Light-elicited currents were analyzed offline in Matlab 2011b/2014b (Mathworks, USA), Igor Pro 6/7 (Wavemetrics, USA) and OriginPro 7 (OriginLab, USA), and this included the correction of baseline drifts during the 10 s-long chirp recordings. For recordings at $36^{\circ} \mathrm{C}$ occasionally spike-like artifacts occurred due to the in-line heater. Those spikes were removed for display purposes in Figure 1.1.

\section{Dissociation and culture of hippocampal neurons, AAV infection and immunocytochemistry}

E18 pregnant Wistar rats were sacrificed using $\mathrm{CO}_{2}$, embryos were removed and heads of embryos were placed in a $10 \mathrm{~cm}$ petri dish containing ice-cold dissection media (HBSS $($ Gibco $)+10 \mathrm{mM}$ Hepes (Gibco)). Brains were removed and collected in fresh dissection medium. Hippocampi were separated from the brain and meninges were removed. Hippocampi were digested with $2 \mathrm{ml}$ pre-warmed $37^{\circ} \mathrm{C} 0.05 \%$ trypsin-EDTA (Gibco, Germany) for $20 \mathrm{~min}$ at $37^{\circ} \mathrm{C}$. Trypsin was removed and the tissue was washed three times with $4^{\circ} \mathrm{C}$ dissection medium. Dissection medium was replaced with $1 \mathrm{ml}$ pre-warmed NB+ (Neurobasal with 1X B-27 supplement, 1X Glutamax and penicillin (5000 $\mathrm{U} \mathrm{ml}^{-}$ $\left.{ }^{1}\right) /$ streptomycin $\left(5000 \mu \mathrm{g} \mathrm{ml}^{-1}\right)$; all from Gibco) and tissue was triturated by gentle pipetting. The tissue suspension was filtered through a $100 \mathrm{~mm}$ cell strainer (BD Biosciences). Cells were counted using the trypan blue exclusion method and cultured on $12 \mathrm{~mm}$ glass coverslips (Thermo Scientific) coated with poly-D-lysine (PDL, Sigma) dissolved in 0.1 M borate buffer, in 24-well plates (CytoOne) at a density of 80000 hippocampal neurons per $\mathrm{cm}^{2}$ in $\mathrm{NB}^{+}$medium in a HeraCell 240i cell culture incubator (Thermo Scientific) at $37^{\circ} \mathrm{C}$ and $5 \%$ $\mathrm{CO}_{2}$. On DIV 10, neurons were infected with $1 \mu \mathrm{l}$ AAV2/6 Chronos, AAV2/6 Chronos-ES/TS or the same constructs packed in PHP.B virus capsid. $72 \mathrm{~h}$ after infection, neurons were briefly washed with pre-warmed PBS and then fixed with $4 \%$ PFA at RT for $10 \mathrm{~min}$. After fixation and several steps of washing, neurons were subjected to immunocytochemistry (rabbit anti-Map2 primary antibody, 1:1000, Abcam and goat anti-rabbit Alexa 555 secondary antibody, 1:1000, Invitrogen) and later on to confocal microscopy (Leica SP5). 


\section{Virus purification}

AAVs were generated in HEK-293T cells (ATCC) using polyethylenimine transfection (25.000 MW, Polysciences, USA) (Gray et al., 2011; Deverman et al., 2016). In brief, triple transfection of HEK-293T cells was performed using pHelper plasmid (TaKaRa/Clontech), trans-plasmid providing viral capsid PHP.B (generous gift from Ben Deverman and Viviana Gradinaru, Caltech, USA) and cis-plasmid providing Chronos or Chronos-ES/TS (Figure 1.2A). The cell line was regularly tested for mycoplasma. We harvested viral particles $72 \mathrm{~h}$ after transfection from the medium and $120 \mathrm{~h}$ after transfection from cells and the medium. Viral particles from the medium were precipitated with $40 \%$ polyethylene glycol 8000 (Acros Organics, Germany) in $500 \mathrm{mM} \mathrm{NaCl}$ for $2 \mathrm{~h}$ at $4^{\circ} \mathrm{C}$ and then after centrifugation at $4000 \mathrm{~g}$ for $30 \mathrm{~min}$ combined with cell pellets for processing. The cell pellets were suspended in $500 \mathrm{mM} \mathrm{NaCl}, 40 \mathrm{mM}$ Tris, $2.5 \mathrm{mM} \mathrm{MgCl} 2, \mathrm{pH} 8$, and $100 \mathrm{U} \mathrm{mL}^{-1}$ of salt-activated nuclease (Arcticzymes, USA) at $37^{\circ} \mathrm{C}$ for $30 \mathrm{~min}$. Afterwards, the cell lysates were clarified by centrifugation at $2000 \mathrm{~g}$ for $10 \mathrm{~min}$ and then purified over iodixanol (Optiprep, Axis Shield, Norway) step gradients (15\%, 25\%, $40 \%$ and $60 \%$ ) (Zolotukhin et al., 1999; Grieger et al., 2006) at 320,006 $\mathrm{g}$ for $2.25 \mathrm{~h}$. Viruses were concentrated using Amicon filters (EMD, UFC910024) and formulated in sterile phosphate-buffered saline (PBS) supplemented with $0.001 \%$ Pluronic F-68 (Gibco, Germany). Virus titers were measured using AAV titration kit (TaKaRa/Clontech) according to manufacturer's instructions by determining the number of DNase I resistant vg using qPCR (StepOne, Applied Biosystems). Purity of produced viruses was routinely checked by silver staining (Pierce, Germany) after gel electrophoresis (Novex ${ }^{\mathrm{TM}}$ 4-12 \% Tris-Glycine, Thermo Fisher Scientific) according to manufacturer's instructions. The presence of viral capsid proteins was positively confirmed in all virus preparations. Viral stocks were kept at $-80{ }^{\circ} \mathrm{C}$ until the experimental day.

\section{Transuterine AAV injection into the cochlea}

For in vivo transduction, anesthesia was induced with a mixture of ketamine and xylazine $\left(0.125 / 5 \mathrm{mg} \mathrm{kg}^{-1}\right)$ and maintained with isoflurane (1\%-2\%). Viral inoculum ( $250 \mathrm{nl}, 4 \mathrm{e}+8$ particles $\mu^{-1}$ ) was microinjected through the uterus into the mouse otocyst from E11.5 to E12.5 as previously described (Brigande et al., 2009; Reisinger et al., 2011). Only the left otocyst of each embryo was injected. The non-injected contralateral ear served as an internal control. AAV2/6_hSyn_Chronos-GFP and AAV2/6_hSyn_Chronos-ES/TS were purchased from the University of North Carolina Vector Core, Chapel Hill, USA. 


\section{Postnatal AAV injection into the cochlea}

Postnatal AAV-injection into scala tympani of the left ear via the round window was performed in P5-P7 wild-type C57BL/6 mice essentially as described (Akil et al., 2012) using AAV-PHP.B viral capsids and hSyn promoter to drive transgenic expression of opsins in SGNs. In brief, under general isoflurane anaesthesia and local analgesia achieved by means of xylocaine, the left ear was approached via a dorsal incision and the round window membrane was identified and gently punctured using a borosilicate capillary pipette that was kept in place to inject approximately $1-1.5 \mu \mathrm{l}$ of AAV2/6_hSyn-Chronos $\left(2.33 \mathrm{E}+12 \mathrm{GC} \mathrm{ml}^{-1}\right)$, AAV2/6_hSyn-Chronos-ES/TS (2.2E+12 $\left.\mathrm{GC} \mathrm{ml}^{-1}\right), \quad$ PHP.B_hSyn-Chronos-GFP $\left(1.07 \mathrm{E}+12 \mathrm{GC} \mathrm{ml}^{-1}\right)$ or PHP.B_hSyn-Chronos-ES/TS-EYFP $\left(2.31 \mathrm{E}+12 \mathrm{GC} \mathrm{ml}^{-1}\right)$. After virus application, the tissue above the injection site was repositioned and the wound was sutured and buprenorphine $\left(0.1 \mathrm{mg} \mathrm{kg}^{-1}\right)$ was applied as pain reliever. Recovery of the animals was then daily tracked. In all experiments, mice were randomly selected for injection. No blinding was possible since injections had to be performed in the left ear leaving the right ear as an internal control. Hence, surgery prior to stimulation needed to be done in the injected ear. Animals were then kept in a $12 \mathrm{~h}$ light/dark cycle, with access to food and water ad libitum. All experiments were done in compliance with the national animal care guidelines and were approved by the board for animal welfare of the University Medical Center Goettingen and the animal welfare office of the state of Lower Saxony. The calculation of animal number was performed prior to starting experiments. We planned to use the Wilcoxon Rank Sum Test and an error probability alpha smaller than 0.05 , a power (1-beta) of 0.95 and effect size depending on the precise experimental protocol.

\section{Immunostaining and imaging of cochlear cryosections}

Cochleae were fixed with $4 \%$ paraformaldehyde in phosphate buffered saline $(1 \mathrm{~h})$. Sections of the cochlea were cryosectioned following 0.12 M EDTA decalcification. After incubation of sections for $1 \mathrm{~h}$ in goat serum dilution buffer $(16 \%$ normal goat serum, $450 \mathrm{mM} \mathrm{NaCl}$, $0.6 \%$ Triton $\mathrm{X}-100,20 \mathrm{mM}$ phosphate buffer, $\mathrm{pH}$ 7.4) primary antibodies were applied over night at $4^{\circ} \mathrm{C}$. The following antibodies were used: chicken anti-GFP (catalog no.: ab13970, Abcam, 1:500), guinea pig anti-parvalbumin (catalog no.: 195004, Synaptic Systems, 1:300). Secondary AlexaFluor-labeled antibodies (goat anti-chicken 488 IgG $(\mathrm{H}+\mathrm{L})$, catalog no.: A11039, Thermo-Fisher Scientific, 1:200; goat-anti guinea pig 568 IgG $(\mathrm{H}+\mathrm{L})$, catalog no.: A1107, Thermo-Fisher Scientific, 1:200) were applied for $1 \mathrm{~h}$ at room temperature. Confocal images were collected using a SP5 microscope (Leica) and processed in ImageJ. Expression 
was considered positive when FP fluorescence in a given cell (marked by parvalbumin) was found to be higher than 3 SD above the background fluorescence of the tissue.

For FP localization analysis line profiles (length: $7.5 \mu \mathrm{m}, 7.5 \mu \mathrm{m}$ and $1.5 \mu \mathrm{m}$ for SGNs, HEK cells and hippocampal neurons, respectively, width: 3 pixels) were centered to the outer edge of the estimated cell membrane. The line profiles were oriented perpendicular to the cell edge. Sample size was 1, 1 and 3 per cell for SGNs, HEK cells and hippocampal neurons, respectively. For membrane/intracellular expression ratio, a maximum peak detection was performed for membranous area (defined positionally on the line profiles as -1 to $1 \mu \mathrm{m},-1$ to $1 \mu \mathrm{m}$ and -0.5 to $0.3 \mu \mathrm{m}$ for SGNs, HEK cells, and hippocampal neurons, respectively) and for intracellular area (defined positionally on the line profiles as 1.1 to $2 \mu \mathrm{m}, 1.1$ to $2 \mu \mathrm{m}$, and 0.4 to $0.5 \mu \mathrm{m}$ for SGNs, HEK cells, and hippocampal neurons, respectively).

\section{STED microscopy}

Images were acquired using an Abberior Instruments Expert Line STED microscope, with excitation lasers at $488 \mathrm{~nm}$ and STED lasers at $595 \mathrm{~nm}, 1 \mathrm{~W}$ using a $1.4 \mathrm{NA} 100 \mathrm{x}$ oil immersion objective, either in confocal or in 2D-STED mode.

\section{Optical stimulation in vivo}

The left bulla was reached using a retroauricular approach and opened to expose the cochlea. A $50 / 200 \mu \mathrm{m}$ optical fiber coupled to a 473-nm laser (MLL-FN-473-100, $100 \mathrm{~mW}$, diode pumped solid state [DPSS]; Changchun New Industry Optoelectronics) was inserted into the cochlea via the round window. Irradiance was calibrated with a laser power meter (LaserCheck; Coherent Inc.).

\section{Auditory brainstem responses}

For stimulus generation and presentation, data acquisition, and off-line analysis, we used a NI System and custom-written Matlab software (The MathWorks, Inc.). Optically-evoked ABRs (oABRs) and acoustically-evoked ABRs (aABRs) were recorded by needle electrodes underneath the pinna, on the vertex, and on the back near the legs. The difference potential between vertex and mastoid subdermal needles was amplified using a custom-designed amplifier, sampled at a rate of $50 \mathrm{kHz}$ for $20 \mathrm{~ms}$, filtered $(300-3000 \mathrm{~Hz})$ and averaged across 1000 and 500 presentations (for oABRs and aABRs, respectively). The first ABR wave was detected semi-automatically with a custom-written Matlab script in which the wave was detected for each trace in a temporal window defined by the user and for which the amplitude was bigger than the average +2 standard deviations. Thresholds were determined by visual 
inspection as the minimum sound or light intensity that elicited a reproducible response waveform in the recorded traces.

\section{Juxtacellular recordings from single putative SGNS}

For auditory nerve recordings, glass microelectrodes ( $50 \mathrm{M} \Omega$ ) were advanced through the posterior end of the anteroventral cochlear nucleus using an Inchworm micro-positioner (EXFO Burleigh, NY, USA) and aimed towards the internal auditory canal. Action potentials were amplified using an ELC-03XS amplifier (NPI Electronic, Tamm, Germany), filtered (300-3000 Hz), digitized (National Instruments card PCIe-6323), analyzed and prepared for display using using custom-written Matlab (The MathWorks, Inc.) software.

When light-responsive fibers were found, $400 \mathrm{~ms}$-long pulse trains at repetition rates $20-1000$ $\mathrm{Hz}$ were presented, leaving $100 \mathrm{~ms}$ inter-train recovery over 20 iterations for each tested rate. Different rates were tested following no particular order, being $20 \mathrm{~Hz}$ the first repetition rate presented across all units. For repetition rates higher or equal to $200 \mathrm{~Hz}$, parameters were computed if the spike probability was equal or superior to $5 \%$. If not, values were set to 0 . Phase-locking was quantified using the vector strength (Goldberg \& Brown, 1969), considering a cycle starting at the onset of a light pulse and ending at the onset of the subsequent pulse, and conforming to the equation: vector strength = $\frac{\sqrt{\left[\sum_{\mathrm{i}=1}^{\mathrm{n}} \cos \theta_{\mathrm{i}}\right]^{2}+\left[\sum_{\mathrm{i}=1}^{\mathrm{n}} \sin \theta_{\mathrm{i}}\right]^{2}}}{\mathrm{n}}$, being $\Theta_{1}, \Theta_{2}, \ldots, \Theta_{n}$ cycle phases in which spikes occurred. The Rayleigh test was used to evaluate the significance of vector strength: if $\mathrm{L}>13.8$, the null hypothesis is rejected at the 0.001 significance level (Hillery and Narins, 1987) and insignificant VS were set to 0 . The spike probability was calculated as the ratio between the number of spikes and the number of light-pulses. The temporal jitter is the standard deviation of spike latency across trials. The hazard function (for the temporal jitter analysis) was calculated for each stimulation rate by simulating spiking as a Poisson process at given rates (from 10 to 1000 spikes s$^{-1}$ ).

\section{Data analysis}

The data were analysed using Matlab (Mathworks), Excel (Microsoft), Igor Pro (Wavemetrics), FIJI (ImageJ2), Origin (Microcal Software), and GraphPad Prism (GraphPad Software). Averages were expressed as mean \pm s.e.m. or mean \pm s.d., as specified. References to data in the main text were expressed as mean \pm s.e.m. For statistical comparison between two groups, data sets were tested for normal distribution (the D'Agostino \& Pearson omnibus normality test or the Shapiro-Wilk test) and equality of variances (F-test) followed by two- 
tailed unpaired Student's t-test, or the unpaired two-tailed Mann-Whitney U test when data were not normally distributed and/or variance was unequal between samples.

For evaluation of multiple groups, statistical significance was calculated by using one-way ANOVA test followed by Tukey's test for normally distributed data (equality of variances tested with the Brown-Forsythe test) or one-way Kruskal-Wallis test followed by Dunn's test for non-normally distributed data.

Acknowledgments: We would like to thank Dr. Edward Boyden for providing the ChronosEGFP construct and Rachel Care for contributing in the initial phase of the project. We thank Andrea Koch, Daniela Gerke, Sandra Gerke and Christiane Senger-Freitag for expert technical assistance and Gerhard Hoch for devising hard- and software for system physiology and Jakob Neef for the help with STED microscopy. We thank Camin Dean for providing hippocampal neuronal culture. We thank Blanche Schwappach for feedback on the manuscript.

Funding: This work was funded by the European Research Council through the Advanced Grant 'OptoHear" to T.M. under the European Union's Horizon 2020 Research and Innovation program (grant agreement No. 670759) and was further supported by the German Ministry of Research and Education through the Bernstein Center for Computational Neuroscience to A.N., F.W. and T.M. (under grant number 01GQ1005A) and the grant Optical CI (under grant number 13N13729) to T.M.) and the German Research Foundation (through the DFG-Research Center (FZT103) and Cluster of Excellence (EXC171) Center for Nanoscale Microscopy and Molecular Physiology of the Brain to T.M., as well as the Leibniz Program to T.M.).

Author contributions: D.K., V.R., F.W., A.N. and T.M. designed the study. D.K. performed o/aABR recordings, immunohistochemistry and FP expression analysis. A.G., T.D. and C.W. performed initial o/aABR recordings. R.M. performed patch-clamp recordings, B.B. performed immunohistochemistry. S.S. and D.L.M. performed initial cloning. V.R. performed AAV injections, HEK293T and hippocampal neuron experiments, confocal and STED microscopy, cloning and production of the viruses. D.L.M. performed recordings from single SGNs and oABRs. A.T.H. developed analysis toolboxes for ABRs and recordings from single SGNs and performed analysis of electrophysiological data. All authors analyzed data and contributed to the writing of the manuscript. T.M., D.K., V.R. and A.N. took a lead on preparing the manuscript.

Competing interests: The authors declare no competing interests. 


\section{Expanded View Figures}

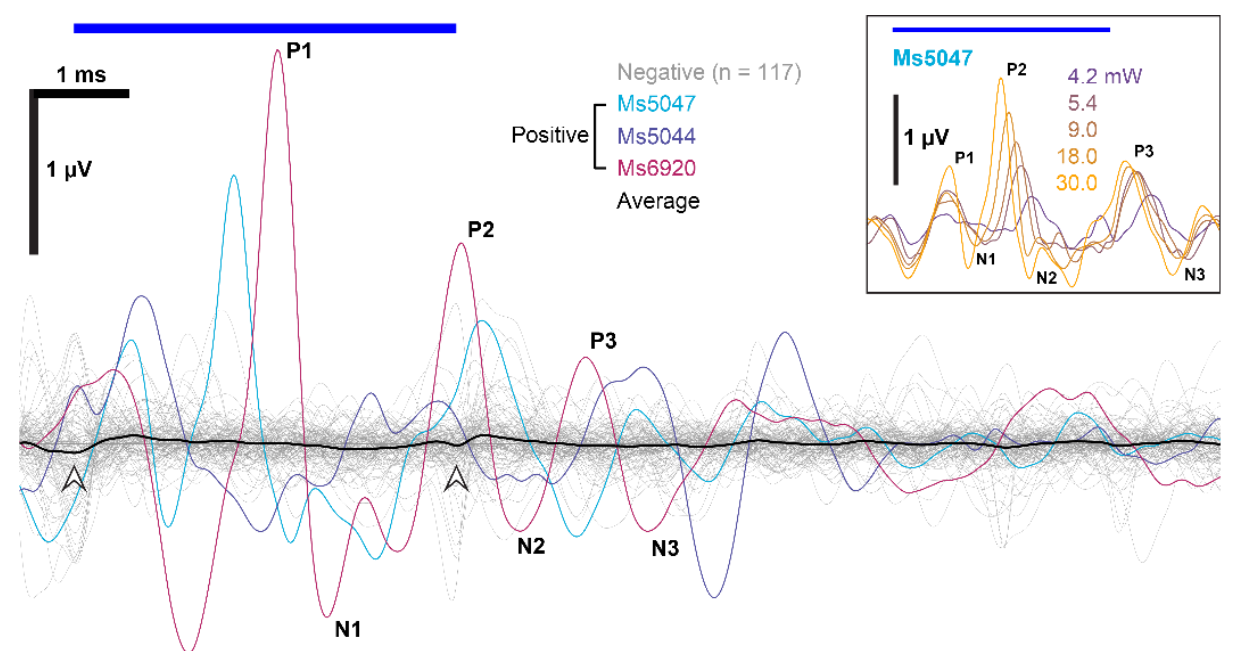

Figure EV1.1: Demonstrating functional expression of Chronos in SGNs following transuterine AAV2/6 injection reported by recordings of oABRs

In 3 out of dozens of animals we could record oABRs using fiber-coupled laser stimulation 412 weeks after embryonic transuterine injection of AAV2/6 carrying Chronos using $4 \mathrm{~ms}$ pulse trains at $10 \mathrm{~Hz}$ with $30 \mathrm{~mW}$ radiant flux. Responses were verified as oABRs by the increasing amplitude with stronger light pulses (inset: shows oABRs of an exemplary oABRpositive mouse for varying radiant flux, colors code the radiant flux in $\mathrm{mW}$ ). A small onset and offset artifact (arrowheads) is visible for the average (black) of the negative animals (gray) which we occasionally observed in the early phase of the project. 
A
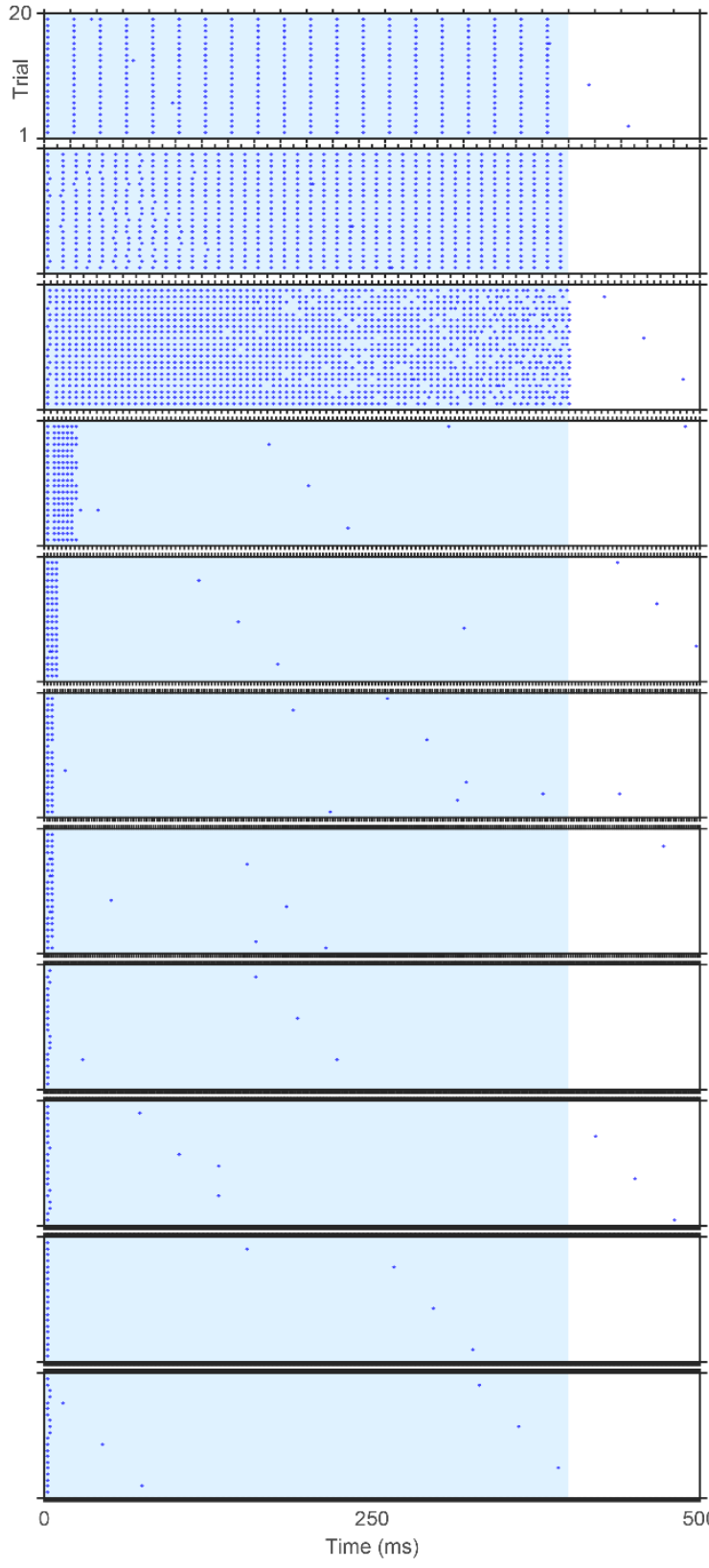

Figure EV1.2: Activity of two exemplary putative SGNs upon light stimulation at increasing repetition rates

A: Raster plot showing spike timing (blue symbols) of a rapidly adapting putative SGN in response to $400 \mathrm{~ms}$ pulse trains at stimulation rates $50-1000 \mathrm{~Hz}(30 \mathrm{~mW}, 1 \mathrm{~ms}$ for $50-600 \mathrm{~Hz}$; $30 \mathrm{~mW}, 0.5 \mathrm{~ms}$ for $700-1000 \mathrm{~Hz}$ ). B: Raster plot showing activity of a slowly adapting putative SGN in response to the stimulation paradigm described in A. 


\section{References and Notes}

Adamantidis A, Arber S, Bains JS, Bamberg E, Bonci A, Buzsáki G, Cardin JA, Costa RM, Dan Y, Goda Y, Graybiel AM, Häusser M, Hegemann P, Huguenard JR, Insel TR, Janak PH, Johnston D, Josselyn SA, Koch C, Kreitzer AC, et al (2015) Optogenetics: 10 years after ChR2 in neurons--views from the community. Nat. Neurosci. 18: 1202-1212

Akil O, Seal RP, Burke K, Wang C, Alemi A, During M, Edwards RH \& Lustig LR (2012) Restoration of Hearing in the VGLUT3 Knockout Mouse Using Virally Mediated Gene Therapy. Neuron 75: 283-293

Boyden ES, Zhang F, Bamberg E, Nagel G \& Deisseroth K (2005) Millisecond-timescale, genetically targeted optical control of neural activity. Nat Neurosci 8: 1263-1268

Brigande JV, Gubbels SP, Woessner DW, Jungwirth JJ \& Bresee CS (2009) Electroporationmediated gene transfer to the developing mouse inner ear. Methods Mol. Biol. 493: 125-139

Busskamp V, Picaud S, Sahel JA \& Roska B (2012) Optogenetic therapy for retinitis pigmentosa. Gene Ther. 19: 169-175

Deverman BE, Pravdo PL, Simpson BP, Kumar SR, Chan KY, Banerjee A, Wu W-L, Yang B, Huber N, Pasca SP \& Gradinaru V (2016) Cre-dependent selection yields AAV variants for widespread gene transfer to the adult brain. Nat. Biotechnol. 34: 204-209

Duarte MJ, Kanumuri VV, Landegger LD, Tarabichi O, Sinha S, Meng X, Hight AE, Kozin ED, Stankovic KM, Brown MC \& Lee DJ (2018) Ancestral Adeno-Associated Virus Vector Delivery of Opsins to Spiral Ganglion Neurons: Implications for Optogenetic Cochlear Implants. Mol. Ther. Available at: http://www.sciencedirect.com/science/article/pii/S1525001618302569 [Accessed July 31, 2018]

Goldberg JM \& Brown PB (1969) Response of binaural neurons of dog superior olivary complex to dichotic tonal stimuli: some physiological mechanisms of sound localization. $J$. Neurophysiol. 32: 613-636

Gradinaru V, Zhang F, Ramakrishnan C, Mattis J, Prakash R, Diester I, Goshen I, Thompson KR \& Deisseroth K (2010) Molecular and Cellular Approaches for Diversifying and Extending Optogenetics. Cell 141: 154-165 
Gray SJ, Choi VW, Asokan A, Haberman RA, McCown TJ \& Samulski RJ (2011) Production of Recombinant Adeno-Associated Viral Vectors and Use in In Vitro and In Vivo Administration. In Current Protocols in Neuroscience, Crawley JN Gerfen CR Rogawski MA Sibley DR Skolnick P \& Wray S (eds) Hoboken, NJ, USA: John Wiley \& Sons, Inc. Available at: http://doi.wiley.com/10.1002/0471142301.ns0417s57 [Accessed January 19, 2018]

Grieger JC, Choi VW \& Samulski RJ (2006) Production and characterization of adenoassociated viral vectors. Nat. Protoc. 1: $1412-1428$

Guo W, Hight AE, Chen JX, Klapoetke NC, Hancock KE, Shinn-Cunningham BG, Boyden ES, Lee DJ \& Polley DB (2015) Hearing the light: neural and perceptual encoding of optogenetic stimulation in the central auditory pathway. Sci. Rep. 5: Available at: http://www.nature.com/srep/2015/150522/srep10319/full/srep10319.html\#supplementaryinformation [Accessed May 29, 2015]

Hernandez VH, Gehrt A, Reuter K, Jing Z, Jeschke M, Mendoza Schulz A, Hoch G, Bartels M, Vogt G, Garnham CW, Yawo H, Fukazawa Y, Augustine GJ, Bamberg E, Kügler S, Salditt T, de Hoz L, Strenzke N \& Moser T (2014) Optogenetic stimulation of the auditory pathway. J. Clin. Invest. 124: 1114-1129

Hight AE, Kozin ED, Darrow K, Lehmann A, Boyden E, Brown MC \& Lee DJ (2015) Superior temporal resolution of Chronos versus channelrhodopsin-2 in an optogenetic model of the auditory brainstem implant. Hear. Res. 322: 235-241

Hillery CM \& Narins PM (1987) Frequency and time domain comparison of low-frequency auditory fiber responses in two anuran amphibians. Hear. Res. 25: 233-248

Hofherr A (2005) Selective Golgi export of Kir2.1 controls the stoichiometry of functional Kir2.x channel heteromers. J. Cell Sci. 118: 1935-1943

Hofherr A, Fakler B \& Klöcker N (2005) Selective Golgi export of Kir2.1 controls the stoichiometry of functional Kir2.x channel heteromers. J. Cell Sci. 118: 1935-1943

Jeschke M \& Moser T (2015) Considering optogenetic stimulation for cochlear implants. Hear. Res. 322: 224-234 
Kim CK, Adhikari A \& Deisseroth K (2017) Integration of optogenetics with complementary methodologies in systems neuroscience. Nat. Rev. Neurosci. 18: 222-235

Klapoetke NC, Murata Y, Kim SS, Pulver SR, Birdsey-Benson A, Cho YK, Morimoto TK, Chuong AS, Carpenter EJ, Tian Z, Wang J, Xie Y, Yan Z, Zhang Y, Chow BY, Surek B, Melkonian M, Jayaraman V, Constantine-Paton M, Wong GK-S, et al (2014) Independent optical excitation of distinct neural populations. Nat. Methods 11: 338-346

Kral A, Hartmann R, Mortazavi D \& Klinke R (1998) Spatial resolution of cochlear implants: the electrical field and excitation of auditory afferents. Hear. Res. 121: 11-28

Lalwani AK, Walsh BJ, Reilly PG, Muzyczka N \& Mhatre AN (1996) Development of in vivo gene therapy for hearing disorders: introduction of adeno-associated virus into the cochlea of the guinea pig. Gene Ther. 3: 588-592

Lenarz T (2018) Cochlear implant - state of the art. GMS Curr. Top. Otorhinolaryngol. Head Neck Surg. 16: Available at: https://www.ncbi.nlm.nih.gov/pmc/articles/PMC5818683/ [Accessed July 31, 2018]

Liberman MC (1978) Auditory-nerve response from cats raised in a low-noise chamber. $J$. Acoust. Soc. Am. 63: 442-455

Ma D, Zerangue N, Lin Y-F, Collins A, Yu M, Jan YN \& Jan LY (2001) Role of ER Export Signals in Controlling Surface Potassium Channel Numbers. Science 291: 316-319

Mager T, Morena DL de la, Senn V, Schlotte J, D’Errico A, Feldbauer K, Wrobel C, Jung S, Bodensiek K, Rankovic V, Browne L, Huet A, Jüttner J, Wood PG, Letzkus JJ, Moser T \& Bamberg E (2018) High frequency neural spiking and auditory signaling by ultrafast redshifted optogenetics. Nat. Commun. 9: 1750

Maguire LP, Szilagyi S \& Scholten RE (2004) High performance laser shutter using a hard disk drive voice-coil actuator. Rev. Sci. Instrum. 75: 3077

Mardinly AR, Oldenburg IA, Pégard NC, Sridharan S, Lyall EH, Chesnov K, Brohawn SG, Waller L \& Adesnik H (2018) Precise multimodal optical control of neural ensemble activity. Nat. Neurosci. 21: 881-893 
Nagel G, Ollig D, Fuhrmann M, Kateriya S, Musti AM, Bamberg E \& Hegemann P (2002) Channelrhodopsin-1: a light-gated proton channel in green algae. Science 296: 2395-2398

Nagel G, Szellas T, Huhn W, Kateriya S, Adeishvili N, Berthold P, Ollig D, Hegemann P \& Bamberg E (2003) Channelrhodopsin-2, a directly light-gated cation-selective membrane channel. Proc. Natl. Acad. Sci. 100: 13940-13945

Reisinger E, Bresee C, Neef J, Nair R, Reuter K, Bulankina A, Nouvian R, Koch M, Buckers J, Kastrup L, Roux I, Petit C, Hell SW, Brose N, Rhee J-S, Kugler S, Brigande JV \& Moser T (2011) Probing the Functional Equivalence of Otoferlin and Synaptotagmin 1 in Exocytosis. J. Neurosci. 31: 4886-4895

Richter C-P, Rajguru SM, Matic AI, Moreno EL, Fishman AJ, Robinson AM, Suh E \& Walsh JT (2011) Spread of cochlear excitation during stimulation with pulsed infrared radiation: inferior colliculus measurements. J. Neural Eng. 8: 056006

RST-001 Phase I/II Trial for Advanced Retinitis Pigmentosa - Full Text View ClinicalTrials.gov Available at: https://clinicaltrials.gov/ct2/show/NCT02556736 [Accessed February 20, 2018]

Sahel J-A \& Roska B (2013) Gene therapy for blindness. Annu. Rev. Neurosci. 36: 467-488

Stockklausner C, Ludwig J, Ruppersberg JP \& Klöcker N (2001) A sequence motif responsible for ER export and surface expression of Kir2.0 inward rectifier $\mathrm{K}(+)$ channels. FEBS Lett. 493: 129-133

WHO (2006) Primary Ear \& Hearing Care Training Resource World Health Organization Wrobel C, Dieter A, Huet A, Keppeler D, Duque-Afonso CJ, Vogl C, Hoch G, Jeschke M \& Moser T (2018) Optogenetic stimulation of cochlear neurons activates the auditory pathway and restores auditory-driven behavior in deaf adult gerbils. Sci. Transl. Med. 10: eaao0540

Zeng FG (2017) Challenges in Improving Cochlear Implant Performance and Accessibility. IEEE Trans. Biomed. Eng. 64: 1662-1664

Zeng F-G, Rebscher S, Harrison WV, Sun X \& Feng H (2008) Cochlear Implants:System Design, Integration and Evaluation. IEEE Rev. Biomed. Eng. 1: 115-142 
Zierhofer CM, Hochmair-Desoyer IJ \& Hochmair ES (1995) Electronic design of a cochlear implant for multichannel high-rate pulsatile stimulation strategies. IEEE Trans. Rehabil. Eng. 3: $112-116$

Zolotukhin S, Byrne BJ, Mason E, Zolotukhin I, Potter M, Chesnut K, Summerford C, Samulski RJ \& Muzyczka N (1999) Recombinant adeno-associated virus purification using novel methods improves infectious titer and yield. Gene Ther. 6: 973-985 


\section{Conclusion and future perspectives}

This study contributes to the field of cochlear optogenetics in two ways for (1) reporting ultrafast and temporally precise light-control of auditory nerve and for (2) improving the opsin of interest. Using a more efficient viral vector and delivery method along with enhanced membrane targeting of Chronos did secure temporally fine-paced activation of the AN fibers. High vector potency and spike probability of the fibers corroborate with presence of wave I in oABR recording at stimulation rates $\geq 500 \mathrm{~Hz}$. Considering encoding of fine temporal structures of sound, this promises high fidelity in bionic coding using optogenetics.

Due to its exceptional physiological relevance, Chronos has a strong stance for future oCI applications. Moreover, blue $\mu$ LED multichannel implants are already available to elicit reliable physiological and behavioral responses in rodents (Dieter et al., 2020; Keppeler et al., 2020; Jablonski et al., 2020). Therefore, one can test better spatial resolution empowered by $\mu$ LED oCIs combined with ultrafast Chronos-mediated temporal resolution of auditory nerve activation in pre-clinical research. The only immediate reservation regarding utility of Chronos in translational setting is chronic stimulation for many years via blue light. The concern lies in the phototoxic effects of blue light in living tissue (Duebel et al., 2015; Sengupta et al., 2016) where short wavelength high-energy photons cause formation of detrimental reactive oxygen species. Additionally, blue light is absorbed and scattered more resulting in poorer tissue penetration. Thus, a quest for longer wavelength channelrhodopsins has begun as red-light stimulation has less of such harmful effects caused by blue one. Yet, is there any red-shifted optogene available just as fast as Chronos? 


\section{Chapter 2: \\ Utility of red-light ultrafast optogenetic stimulation of the auditory pathway}

\section{Synopsis}

Traditionally, blue light-gated channelrhodopsins have often remained the first choice for the optogenetic control of neurons. Depending on the research question, one should also consider modifying the optogene of choice. As described in the previous chapter for example, Chronos, the ultrafast member of the blue-shifted opsins, shows fine temporal orchestration of the optical encoding when efficient membrane localization is achieved. This works well in acute experiments spanning maximum 10-12 hours of blue-light stimulation. Meantime, behavioral experiments using gerbils and rats expressing $\mathrm{CatCh}$, a $\mathrm{ChR} 2$ variant, that can generate reliably detectable percepts over months, though not continuously, by blue-light stimulation (Jablonski et al., 2020; Keppeler et al., 2020; Wrobel et al., 2018). Here, durable SGNs are at utmost importance to provide a long-lasting audition through both eCI and prospective medical oCI systems as well. However, from the translational point of view when implemented in humans, blue light stimulation of the auditory nerve over many years may be harmful and shorten its viability (Kleinlogel et al., 2020). Even the neural tissue of mammalian eye which has evolved to process light will disintegrate faster under long-term blue light exposure (European Commission, 2006).

Blue light bears three disadvantages when considering clinical translatio: (1) reactive oxygen species (ROS) generation, (2) higher absorption and (3) scattering within tissue. First, due to their high-energy nature, photons of blue light can undertake chemical reactions which excessively yield ROS in the cells. These toxic compounds then accumulate over time interfering with cell's equilibrium and eventually cause its death. Secondly, greater absorption of blue light through the surrounding tissue and fluids can diminish the amount of available light for the target neurons in deeper layers. For example, in oCIs, the light emitted should cross perilymph-filled scala tympani and bony capsule until it reaches the most external SGNs wrapped in glial cells. Since penetration of short wavelength light is attenuated, this might in turn decrease the number of SGNs activated at a given light radiant flux or require higher radiant flux, and therefore energy, to stimulate the SGNs. As a third drawback, stronger scattering of blue light might limit the spectral selectivity of the oCI. Red light stimulation, in contrast, avoids such complications since longer wavelength photons contain less energy and 
can penetrate tissue deeper. Hence, these reasons urged us to seek for a red-shifted and still fast channelrhodopsin alternatives.

In the course of discovering new ChRs, besides Chronos, Klapoetke and fellows (2014) also identified a cation-selective channelrhodopsin from Chlamydomonas noctigama having a spectral peak at $590 \mathrm{~nm}$; thus it is named as Chrimson referring to orange-red color. Initial characterization of the channel revealed that Chrimson confers favorably large light-induced currents and hence high light-sensitivity. In terms of activation/deactivation kinetics, on the other hand, it is rather a slow opsin, i.e. $\tau$ on and $\tau$ off are around $6 \mathrm{~ms}$ and $21 \mathrm{~ms}$, respectively, which will not allow high fidelity sustained spiking at ultrafast stimulation rates. In the same article, after substituting a lysine residue to an arginine (K176R), the authors generates a faster mutant ChrimsonR which has a $\tau$ off of $15.8 \pm 0.4 \mathrm{~ms}$ enabling red-light driven precise spiking at least at $20 \mathrm{~Hz}$ stimulation rate; whereas, native one can only allow this up to $10 \mathrm{~Hz}$. The authors, on a final note, state that despite being sufficient for two-color stimulation in mouse cortical neurons, Chrimson needs to be further engineered to speed up the kinetics being as fast as Chronos.

Since the foundation of optogenetics in neurosciences (Boyden et al., 2005), there is an endeavor to discover and devise new optogenes to be able to run fast-spiking neurons with precision. As discovery is more a matter of chance, designing fast channelrhodopsins can provide rapid solutions. The study from Mager and colleagues in 2018 exemplifies such a situation where targeted point mutations are introduced in the protein coding sequence of helix F of Chrimson singly or in combination with others. As described in previous studies (Luecke, 2001; Nakanishi et al., 2013; Subramaniam et al., 1993), closed to open state is achieved when helix F of serpentine opsin molecule moves setting on-/off-cycle time. Among several modified opsins, two become prominent in terms of fast kinetics: (1) double mutant (Y261F/S267M) on helix F nicknamed as fast-Chrimson (f-Chrimson) and (2) triple mutant (K176R/Y261F/S267M), a combination of ChrimsonR (Klapoetke et al., 2014) and helix F mutations, named as very-fast-Chrimson (vf-Chrimson). At body temperature, the f-Chrimson has a closing time constant of $3.2 \mathrm{~ms}$ whereas vf-Chrimson is two folds faster. In addition to accelerating closing kinetics, neither f-Chrimson nor vf-Chrimson has changed optimal absorption wavelength at $590 \mathrm{~nm}$. Motivated by fast kinetics and red action spectrum, the authors then tested both variants in different in vitro and in vivo neuronal scenarios. In hippocampal neuronal cultures, high fidelity and fast spiking was obtained up to $100 \mathrm{~Hz}$ stimulation rate using either variant. Light sensitivities of Chrimson and the variants differed 
from each other such that the slower, the more sensitive. As it can be inferred from the closing time constants, both variants have the potential to drive neurons faster than $100 \mathrm{~Hz}$. Hence, to investigate ultrafast red-shifted neuronal control, f-Chrimson was tested in the auditory nerve in mouse and vf-Chrimson in the fast-spiking parvalbumin-positive neurons in culture. FChrimson enabled optical driving of AN at 200-300 Hz stimulation rate meanwhile requiring low light intensities. Vf-Chrimson however could carry out reliable spiking up to $300 \mathrm{~Hz}$ and above.

A candidate opsin should meet three criteria for hearing via oCI. First, to stimulate fast spiking of auditory nerve fibers the candidate opsin should have rapid on-/off-cycles. Second, it should convey high light sensitivity allowing recruitment of SGNs to encode increasing sound intensities with a reasonable energy budget. Third, chronic red-light stimulation is more favorable than blue considering phototoxicity. The initial study (Mager et al., 2018) brought in f-Chrimson and vf-Chrimson as strong candidates checking the three criteria. What remained unanswered in that study are in vivo characterization of vf-Chrimson focusing on temporal properties and light intensity encoding by f-Chrimson or vf-Chrimson in mouse auditory nerve. To address these, we investigated response properties of mouse auditory nerve upon light stimulation after optogenetic manipulation of cochlea using vf-Chrimson. We also harnessed the viral and molecular developments tested in the Chronos study (Keppeler et al., 2018) to enhance expression level and membrane targeting of vf-Chrimson. 


\section{Manuscript Information}

\section{Utility of red-light ultrafast optogenetic stimulation of the auditory pathway}

Burak Bali ${ }^{1,2,3}$, David Lopez de la Morena ${ }^{1,2,4 \#}$, Artur Mittring ${ }^{1}$, Antoine Tarquin Huet ${ }^{1,4}$, Thomas Mager ${ }^{1,6}$, Vladan Rankovic ${ }^{1,3^{*}}$, Tobias Moser ${ }^{1,4,5,6^{*}}$

\#, *These authors contributed equally to the work

EMBO Mol Med, Under revision

Detailed author contribution of Burak Bali:

- Experimental work and analysis:

$\circ$ Confocal imaging and expression/density analysis of cochlear cryosections (Fig 1)

○ Optically-evoked auditory brainstem recordings (Fig 2)

○ Optically-evoked single unit measurements at the auditory nerve (Fig $3 \& 4$ )

- Preparation of the manuscript (together with the other authors) 


\section{Manuscript}

\section{Utility of red-light ultrafast optogenetic stimulation of the auditory pathway}

Keywords: channelrhodopsin, gating, spiral ganglion, temporal coding, dynamic range, cochlear implant

Running title: Ultrafast red-light cochlear optogenetics

Authors: Burak Bali ${ }^{1,2,3 \#}$, David Lopez de la Morena ${ }^{1,2,4 \#}$, Artur Mittring ${ }^{1}$, Antoine Tarquin Huet $^{1,4}$, Thomas Mager ${ }^{1,6}$, Vladan Rankovic ${ }^{1,3^{*}}$, Tobias Moser ${ }^{1,4,5,6^{*}}$

\section{Affiliations:}

${ }^{1}$ Institute for Auditory Neuroscience and InnerEarLab, University Medical Center Göttingen, Germany

${ }^{2}$ Göttingen Graduate School for Neurosciences and Molecular Biosciences, University of Göttingen, Germany

${ }^{3}$ Restorative Cochlear Genomics group, Auditory Neuroscience and Optogenetics laboratory, German Primate Center, Göttingen, Germany

${ }^{4}$ Auditory Neuroscience and Optogenetics laboratory, German Primate Center, Göttingen, Germany

${ }^{5}$ Auditory Neuroscience Group, Max-Planck-Institute for Experimental Medicine, Göttingen, Germany

${ }^{6}$ Cluster of Excellence "Multiscale Bioimaging: from Molecular Machines to Networks of Excitable Cells" (MBExC), University of Göttingen, Germany

\# equal contribution

*To whom correspondence should be addressed:

Dr. Vladan Rankovic, Email: vrankovic@dpz.eu

Dr. Tobias Moser, Email: tmoser@gwdg.de 


\begin{abstract}
Optogenetic stimulation of spiral ganglion neurons (SGNs) in the ear provides a future alternative to electrical stimulation used in current cochlear implants. Here, we employed fast and very fast variants of the red-light activated channelrhodopsin (ChR) Chrimson (fChrimson and vf-Chrimson) to study their utility for optogenetic stimulation of SGNs of mice. Moreover, we investigated the effects of adding trafficking sequences derived from Kir2.1 to vf-Chrimson. Optogenetic time and intensity coding by single SGN was compared to coding of acoustic clicks. vf-Chrimson enabled SGN to fire at near physiological rates with good temporal precision up to $250 \mathrm{~Hz}$ of stimulation. The dynamic range of optogenetic stimulation was narrower than for acoustic clicks but larger than that reported for electrical stimulation. In contrast to the fast gating blue ChR Chronos, the trafficking sequences did not lend a major benefit for the utility of vf-Chrimson. In conclusion, f-Chrimson and vfChrimson are promising candidates for optogenetic stimulation of SGNs in auditory research and future cochlear implants.
\end{abstract}




\section{Introduction}

Optogenetics, the control of cells with light, has revolutionized the life sciences and bears potential for innovative therapies such as for sensory restoration (Sahel \& Roska, 2013; Kleinlogel et al, 2020; Dieter et al, 2020; Dombrowski et al, 2019). AAV-mediated optogenetics for vision restoration has recently entered clinical trials (ClinicalTrials.gov identifier: NCT02556736, Allergan; NCT03326336, GenSight Biologics) while the development of optogenetic hearing restoration is at the preclinical stage. Restoring light sensitivity of the retina by optogenetics seems plausible and can likely be achieved with ChRs that deactivate within several milliseconds (Busskamp et al, 2012). The unmet need for means to restore vision is very high: except for gene therapy of Leber's congenital amaurosis 2 by Luxturna, an FDA-approved AAV-therapy, currently there are no treatment options for retinal degeneration. As a matter of fact, production of retinal implants has recently stopped in Europe and the US.

Optogenetic hearing restoration, on the other hand, seems less intuitive and rehabilitation of the deaf has successfully built on the cochlear implant (CI). The CI enables open speech comprehension in most of the more than 700,000 users and is, therefore, considered the most successful neuroprosthesis (Zeng, 2017; Lenarz, 2018). However, an urgent need for further improvement of the CI remains: users typically cannot understand speech in the presence of background noise, interpret the emotional tone in speech or appreciate music. As for the retinal implant, the biggest bottleneck of the CI is the wide spread of current around each electrode contact, which limits the spectral resolution of sound coding (Kral et al, 1998). Using light for stimulation in future optical CIs (oCI) is one of the present developments to improve spectral coding by CIs, as light can be better spatially confined than electric current (Hernandez et al, 2014; Richter et al, 2011; Dieter et al, 2019, 2020; Keppeler et al, 2020). However, unlike for vision restoration, high temporal fidelity of bionic sound coding is as important as high spectral resolution of coding that is offered by optical stimulation. Therefore, fast-closing ChRs have been employed, such as Chronos (Keppeler et al, 2018; Duarte et al, 2018) and fast (f-) Chrimson (Mager et al, 2018), which enabled SGN firing at near physiological firing rates. The deactivation time constants of Chronos, very fast (vf)Chrimson and f-Chrimson at physiological temperature amounted to $0.8,1.6$, and $3.2 \mathrm{~ms}$ (Mager et al, 2018; Keppeler et al, 2018). Optogenetic SGN stimulation mediated by Chronos required highly potent vectors (Keppeler et al, 2018; Duarte et al, 2018) and optimization of trafficking (Keppeler et al, 2018). This indicates that short channel lifetimes need to be offset 
by high levels of plasma membrane expression for comparable photo-depolarization at the same light intensity.

f-Chrimson, on the other hand, mediated large photocurrents and enabled optogenetic stimulation of the auditory pathway at lower light intensities (Mager et al, 2018) than those required for Chronos (Keppeler et al, 2018). In fact, the radiant flux threshold for evoking auditory brainstem responses (oABRs) was an order of magnitude lower $(0.5 \mathrm{~mW})$ than for trafficking-optimized Chronos (6.6 mW vs. $14 \mathrm{~mW}$ for the original Chronos). Chrimson variants, as red-light activated ChRs, have the additional advantage of neural stimulation with lower risk of phototoxicity and, therefore, are candidates for translating optogenetic sensory restoration to the clinic (Kleinlogel et al, 2020). Here, we studied the utility of f-Chrimson and vf-Chrimson for optogenetic SGN stimulation addressing temporal fidelity as well as intensity coding. We demonstrate that they enable fast SGN stimulation graded over $>10 \mathrm{~dB}$ $(\mu \mathrm{J})$. 


\section{Results}

Establishing, characterizing and optimizing the utility of $v$-Chrimson-mediated SGN stimulation

In vitro biophysical characterization (Mager et al, 2018) had indicated vf-Chrimson as a strong candidate for optogenetic stimulation of SGNs with high temporal fidelity. As the closing kinetics of vf-Chrimson is twice as fast as that of f-Chrimson (Mager et al, 2018), we anticipated an improved temporal fidelity of SGN stimulation. However, we reasoned that the trafficking of vf-Chrimson to the plasma membrane is less efficient, as the photocurrent density estimated in NG cells (neuroblastoma and neuroglioma cell line, NG108-15) for vfChrimson was not only halved, as one would expect from the closing kinetics being twice faster, but dropped to about one fourth of that of f-Chrimson (Mager et al, 2018). Therefore, we aimed to improve membrane targeting by adding ER-export and trafficking signals, isolated from a vertebrate inward rectifier potassium channel (Stockklausner et al, 2001; Hofherr et al, 2005) sandwiching EYFP (Figure 2.1A). These sequences, nick-named ES (Export Signal) and TS (Trafficking Signal) were shown to enhance the plasma membrane expression of opsins (Keppeler et al, 2018; Gradinaru et al, 2010). We first compared expression of vf-Chrimson-ES/TS and vf-Chrimson in mouse SGNs in vivo. We used local intracochlear injection on postnatal day 6 (P6) of AAV-PHP.B (vf-Chrimson) or AAVPHP.eB (vf-Chrimson-ES/TS) as potent viral vectors (Deverman et al, 2016; Chan et al, 2017; Keppeler et al, 2018) with similar titers, 8.7E+12 and 1.1E+13 genome copies/ml, respectively. We employed the human synapsin promoter (hSyn, Figure 2.1A) that drives efficient and specific channelrhodopsin expression in SGNs (Hernandez et al, 2014; Mager et al, 2018; Wrobel et al, 2018). In order to evaluate viral transduction and membrane expression of vf-Chrimson-ES/TS and vf-Chrimson in SGNs, we performed confocal imaging of mid-cochlear sections following decalcification and immunolabeling for parvalbumin (SGN marker) and EYFP (for detection of the opsins). 
A

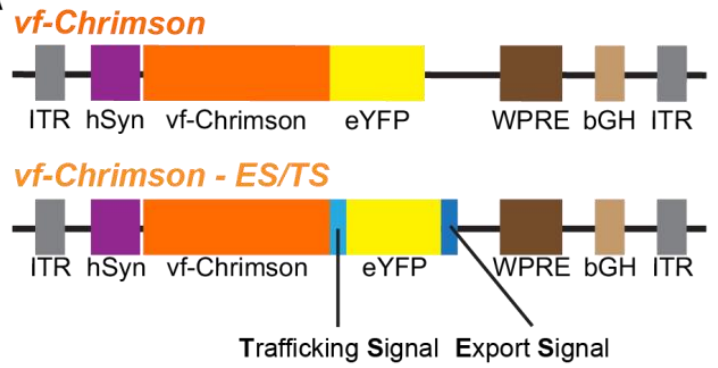

$\mathrm{Bi}$

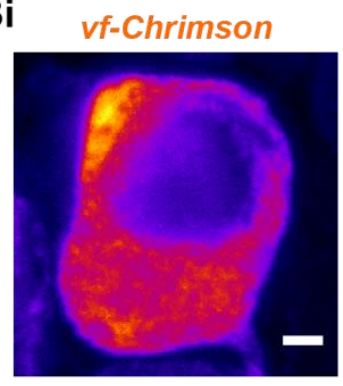

Bii

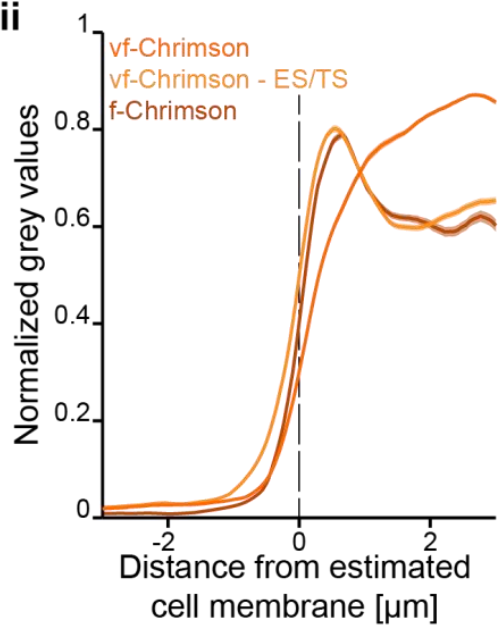

D
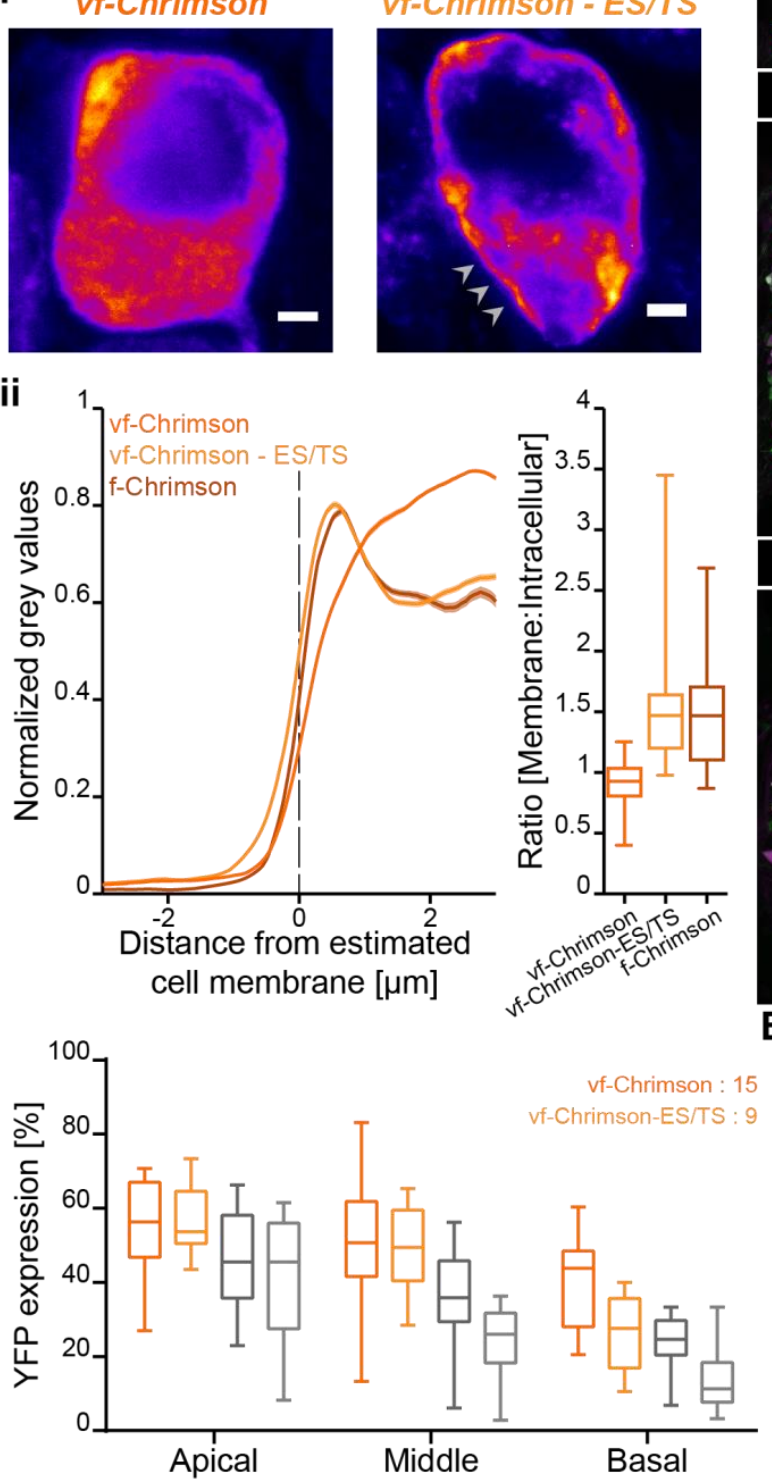
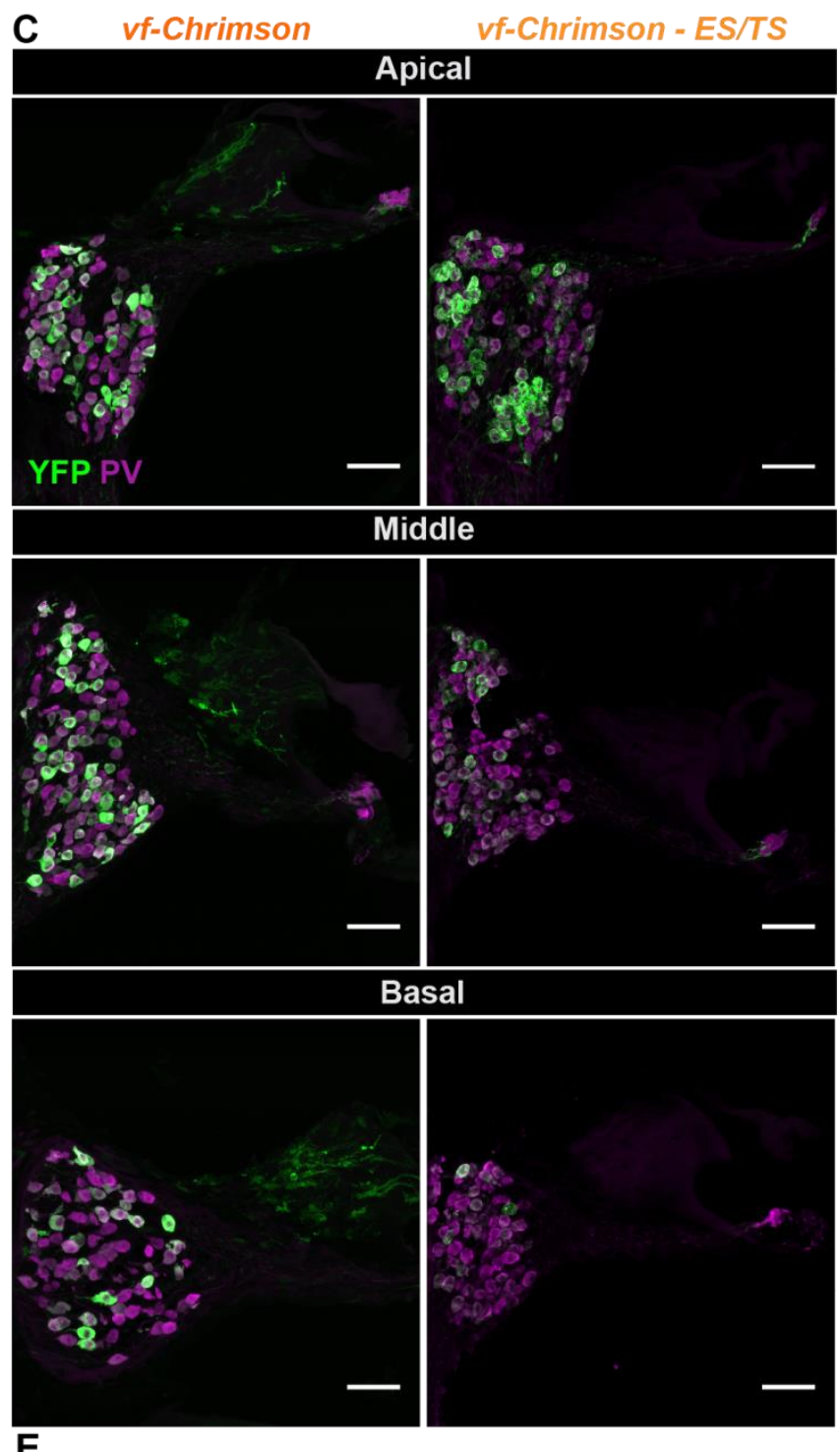

$\mathbf{E}$

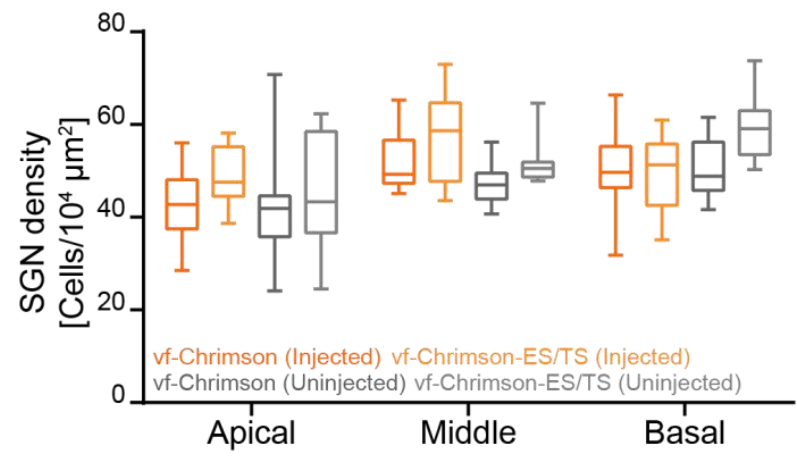

Figure 2.1. Establishing efficient expression of vf-Chrimson in SGNs

(A) pAAV vector used in the study containing vf-Chrimson-EYFP ("vf-Chrimson", upper) or with a trafficking signal (TS), EYFP and ER export signal (ES) vf-Chrimson-EYFP ("vfChrimson-ES/TS", lower). In each, expression was driven by the human synapsin promoter (hSyn) and enhanced by the Woodchuck hepatitis virus posttranslational regulatory element (WPRE) and bovine Growth Hormone (bGH) polyadenylation signal (bGH poly A) sequences. ITR: inverted terminal repeats.

(B)(i) Single-stack confocal images of GFP-immunolabeled SGN somata transduced by either construct are shown. Warmer colors represent higher fluorescence intensity finely indicating subcellular distribution of the channelrhodopsin expression. Enhanced localization of the 
opsin to the plasma membrane (arrowheads) is clearly visible when employing the trafficking signals. Scale bars: $2 \mu \mathrm{m}$. (ii) Quantification for the enhanced membrane localization of vfChrimson is done as previously described (Keppeler et al., 2018) where fluorescence intensity of the immunofluorescence of the anti-GFP-antibody (mean \pm SEM) is plotted across the cell membrane ( $\mathrm{n}=30$ cells, $\mathrm{N}=5$ mice per group). In comparison, line profile of $\mathrm{f}$-Chrimson is analyzed from Mager et al., 2018, which innately favors localization at the plasma membrane. Right panel: Box-and-whisker plots of ratio of maximum membrane fluorescence to maximum cytoplasm one are shown. The vf-chrimson without the trafficking tags score around 1, which is lower compared to the other two ( $\mathrm{p}<0.0001$, Kruskal-Wallis test, Dunn's multiple comparison test) suggesting a relatively ubiquitous intracellular distribution of the opsin.

(C) Representative maximum-projection confocal images of fluorescently-labeled midmodiolar cryosections of injected mouse cochlea are shown for both constructs. SGN somata (magenta) and transduced neurons (green) are labeled along the modiolar cross sections. Scale bars: $50 \mu \mathrm{m}$.

(D) Box-and-whisker plots of the percentage of GFP-positive SGNs to all PV-positive ones are shown for the three modiolar turns of the mouse cochlea which is either injected (colored) or contralateral non-injected (grey). The horizontal line within the box indicates the median, boundaries of the box indicate the 0.25- and 0.75-percentile, and the whiskers indicate the highest and lowest values of the results. No significant differences are observed between vfChrimson and vf-Chrimson-ES/TS injected ears $(\mathrm{N}=15$ for vf-Chrimson; $\mathrm{N}=9$ for vfChrimson-ES/TS; $\mathrm{p}=0.3596$, Mann-Whitney-U test).

(E) Box-and-whisker plots of the SGN density (number of PV-positive SGN somata per cross-sectional area of Rosenthal's canal) are shown for the three turns of the mouse cochlea either injected (colored) or contralateral uninjected (grey). No significant differences are observed between vf-Chrimson and vf-Chrimson-ES/TS in the injected ear $(p=0.1060$, Mann-Whitney test) nor between injected and non-injected cochleae of either construct $(\mathrm{N}=$ 15 for vf-Chrimson; $\mathrm{N}=9$ for vf-Chrimson-ES/TS; $\mathrm{p}$ (vf-Chrimson) $=0.2157$; $\mathrm{p}$ (vfChrimson-ES/TS) $=0.6517$, Mann-Whitney-U test).

First, we probed for membrane targeting of vf-Chrimson and vf-Chrimson-ES/TS (Figure 2.1B). The ratio of membrane over intracellular immunofluorescence obtained from line profile analysis was significantly larger for vf-Chrimson-ES/TS than for vf-Chrimson, and similar to f-Chrimson (Figure 2.1 Bii) and to Chronos-ES/TS (Keppeler et al, 2018). Expression of vf-Chrimson-ES/TS and vf-Chrimson was found across all turns of the injected cochlea (Figure 2.1C) with overall transduction rate of approximately 50\% and a tendency for higher rates of expression in apical SGNs in both cases (Figure 2.1D, apical vs. basal, p = 0.0014, Mann-Whitney-U test). We also found substantial expression in the contralateral, non-injected cochleae, indicating spread of virus in the specific conditions of pressure injection into the scala tympani of the early postnatal cochlea (Figure 2.1D). This spread likely occurred via the cochlear aqueduct and/or the endolymphatic ducts and the cerebrospinal fluid space (Lalwani et al, 1996). Clearly, incomplete and inhomogeneous SGN transduction along the tonotopic axis as well as viral spread indicate the need for further optimization of AAV-mediated gene transfer for improved efficiency and safety. The 
densities of SGNs in the injected as well as in non-injected ears were not significantly different from each other (Figure 2.1E). The injected mice behaved normally as concluded from routine animal observation and lacked obvious phenotypes such as circling, seizures, abnormal motor activity or reduced body size.

oABRs were recorded in response to $594 \mathrm{~nm}$ light delivered via a $200 \mu \mathrm{m}$ optical fiber that was inserted into the round window. oABRs were found in 9 out of 11 AAV-PHP.eB-vfChrimson-ES/TS-injected mice and 13 out of 16 AAV-PHP.B-vf-Chrimson-injected mice. Please note that the negative deflection of the signal before stimulus onset results from lowpass filtering of the oABRs. oABRs of AAV-PHP.eB-vf-Chrimson-ES/TS-injected mice typically showed more differentiated waveforms, i.e. displaying more obvious ABR waves beyond wave I than those of AAV-PHP.B-vf-Chrimson-injected mice (Figure 2.2A, $1 \mathrm{~ms}$ light pulses delivered at $10 \mathrm{~Hz}, 1000$ repetitions). In both cases, the amplitude of oABR wave $\mathrm{I}, \mathrm{P}_{1}-\mathrm{N}_{1}$, reflecting the optically evoked spiral ganglion compound action potential, grew with increasing stimulus intensity (Figure 2.2D) while its latency (i.e. the time interval between the stimulus onset and the oABR $\mathrm{P}_{1}$ wave) got shorter (Figure 2.2E). 
A

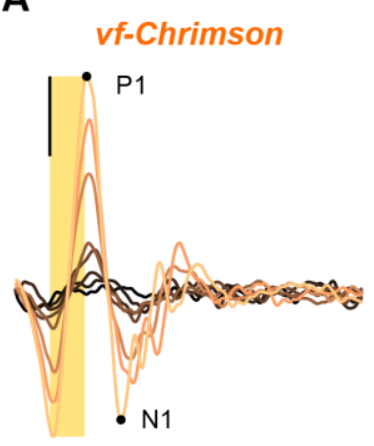

B

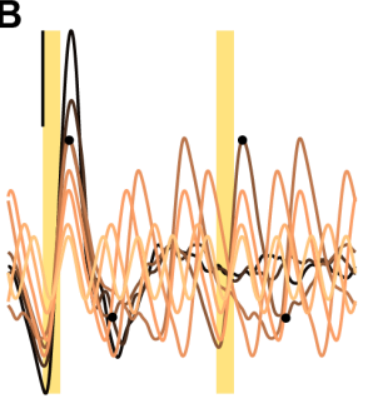

C

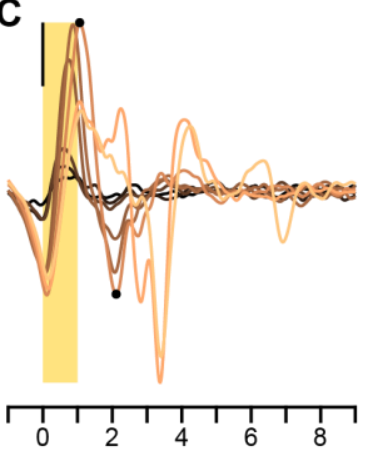

Time to pulse onset [ms]
vf-Chrimson - ES/TS
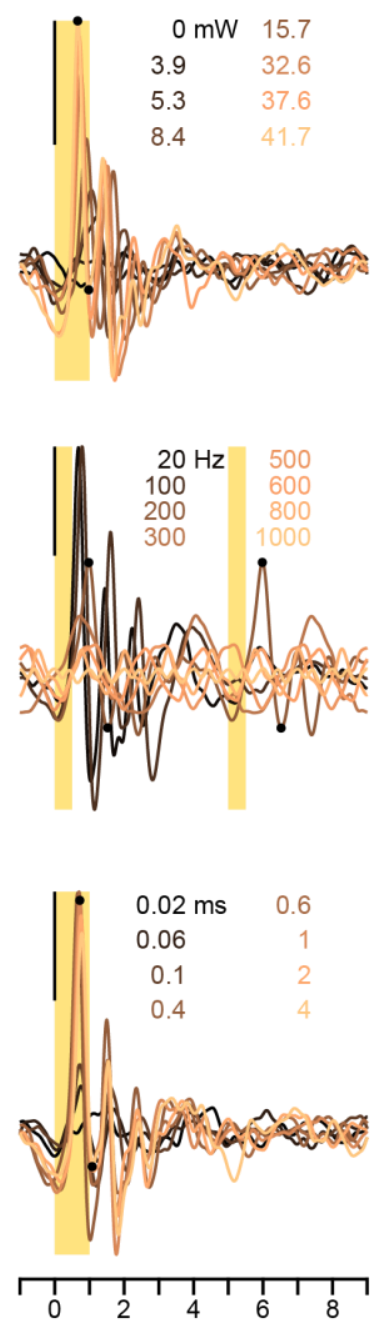

Time to pulse onset [ms]

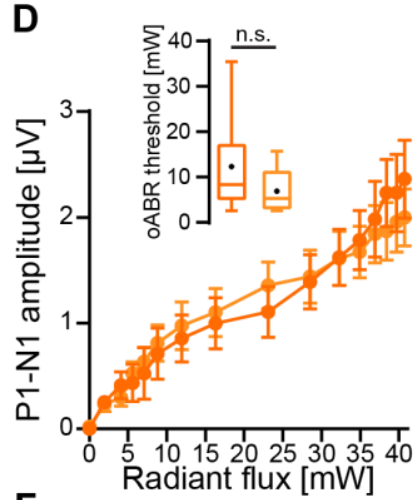

$\mathbf{F}$

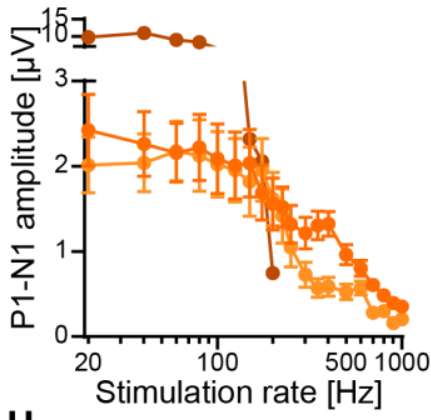

$\mathbf{H}$

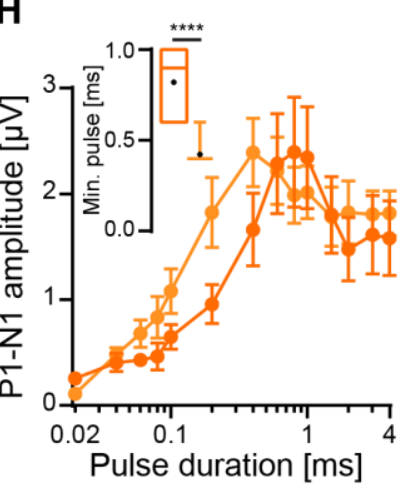

E

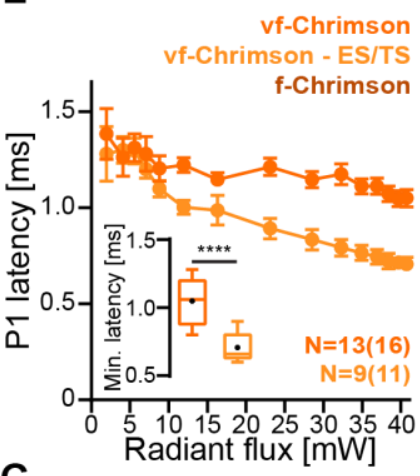

G
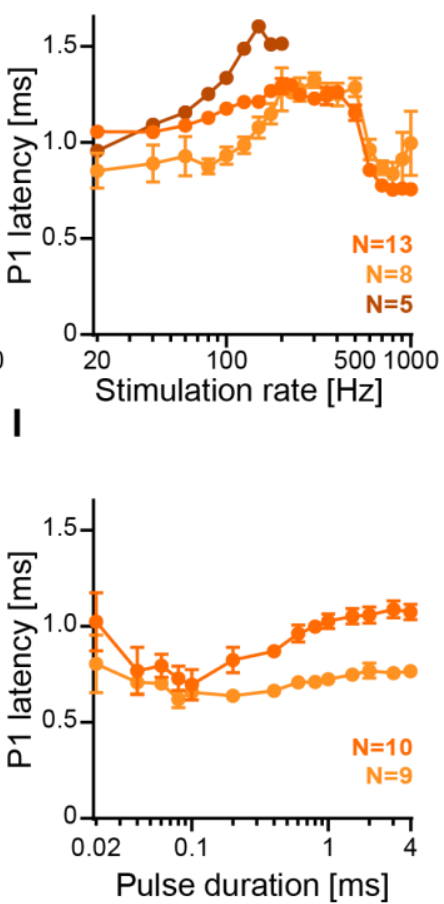

Figure 2.2. Characterizing optogenetic stimulation by recordings of oABRs

(A) Exemplary oABRs driven with varying radiant flux $(1 \mathrm{~ms}$ at $10 \mathrm{~Hz}$, colors code the radiant flux in $\mathrm{mW}$ ) of mice injected with AAV-PHP.B-vf-Chrimson (left) or AAV-PHP.Bvf-Chrimson-ES/TS (right). First positive (P1) and first negative (N1) peaks are indicated by black dots on the waveform triggered upon $41.7 \mathrm{~mW}$ radiant flux (the initial negative deflection results from filtering). Vertical scale bar: $1 \mu \mathrm{V}$.

(B) Exemplary oABRs driven with varying stimulation rate ( $1 \mathrm{~ms}$ for data points $\leq 500 \mathrm{~Hz}$ and $0.5 \mathrm{~ms}$ above $500 \mathrm{~Hz}$ at $41.7 \mathrm{~mW}$ (highest radiant flux), colors code the stimulation rate) of the same animals from (A). Stimuli applied at a rate of $200 \mathrm{~Hz}$ and the corresponding P1-N1 pairs are indicated by yellow shaded area and black dots, respectively. Vertical scale bar: 1 $\mu \mathrm{V}$.

(C) oABRs driven with varying light pulse duration $(10 \mathrm{~Hz}$ at $41.7 \mathrm{~mW}$ (highest radiant flux)), colors code the pulse duration) of the same animals from (A). Exemplary pulse duration of $1 \mathrm{~ms}$ and corresponding P1-N1 pair are indicated by yellow shaded area and black dots. Vertical scale bar: $1 \mu \mathrm{V}$.

$(\mathrm{D}, \mathrm{F}, \mathrm{H})$ plot P1 - N1 amplitudes (mean \pm SEM) of oABR as a function of radiant flux (D), stimulation rate $(\mathrm{F})$, and pulse duration $(\mathrm{H})$. The box-and-whisker plot $(\mathrm{D}$, inset) represents the distribution of observed oABR thresholds of each animal where the black dot is the mean and horizontal line is for median threshold value. A similar plot in $(\mathrm{H}$, inset) represents the 
minimum pulse duration required to elicit maximum P1-N1 amplitude. The average P1-N1 amplitude of f-Chrimson (brown in F and G) is replotted from Mager et al., 2018.

(E, G, I) P1 latencies (mean \pm SEM) of oABR as a function of radiant flux $(\mathrm{E})$, stimulation rate $(\mathrm{G})$, and pulse duration (I) are plotted. The box-and-whisker plot (E, inset) represents the distribution of shortest P1 latencies elicited among any radiant flux. P1 latencies were significantly shorter for vf-Chrimson-ES/TS than for vf-Chrimson $(p<0.0001$, MannWhitney-U test).

On average, the oABR threshold for radiant flux amounted to $6.88 \pm 1.72 \mathrm{~mW}$ ( $\mathrm{N}=9$ mice) for vf-Chrimson-ES/TS and $12.3 \pm 2.81 \mathrm{~mW}(\mathrm{~N}=13 \mathrm{mice}$ ) for vf-Chrimson (duration: $1 \mathrm{~ms}$, rate: $10 \mathrm{~Hz}, 1000$ repetitions, $\mathrm{p}=0.1191$, Mann-Whitney-U test). In most animals, the growth of oABR amplitudes did not saturate with increasing radiant flux, despite changes over more than an order of magnitude (Figure 2.2D). The minimal latency of the first oABR peak $\left(\mathrm{P}_{1}\right.$ latency at the maximum radiant flux, Figure 2.2E inset) was shorter for vf-Chrimson-ES/TS $(0.71 \pm 0.03 \mathrm{~ms}, \mathrm{~N}=9)$ than for vf-Chrimson-mediated oABRs $(1.05 \pm 0.05 \mathrm{~ms}, \mathrm{~N}=13, \mathrm{p}<$ 0.0001, Mann-Whitney-U test). Next, we tested the dependence of oABRs on the stimulus rate (duration: $1 \mathrm{~ms}$ up $500 \mathrm{~Hz}$, intensity: maximum $(38-43 \mathrm{~mW})$ ). When increasing stimulus rate, oABR amplitudes declined (Figure 2.2F) and latencies increased (Figure 2.2G). However, we could detect sizable $\mathrm{P}_{1}-\mathrm{N}_{1}$ up to stimulus rates of $500 \mathrm{~Hz}$. Amplitudes were comparable between vf-Chrimson and vf-Chrimson-ES/TS for stimulation rates of 100, 300 and $500 \mathrm{~Hz}$ ( $\mathrm{p}>0.05$, Kruskal-Wallis ANOVA, post-hoc Dunn's multiple comparison test). For a reference we replot the data from f-Chrimson-expressing SGNs recorded in our previous studies (Mager et al, 2018). Sizable oABRs could be elicited by light pulses as short as $20 \mu \mathrm{s}$ with vf-Chrimson and $40 \mu \mathrm{s}$ with vf-Chrimson-ES/TS (irradiance: $38 \mathrm{~mW}$, rate: $10 \mathrm{~Hz}-$ averaged across 1000 repetitions, Figure 2.2C). oABR amplitudes grew with pulse duration up to approximately $0.4 \mathrm{~ms}$ for vf-Chrimson-ES/TS and $0.8 \mathrm{~ms}$ for vf-Chrimson ( $\mathrm{p}<0.0001$, Mann-Whitney-U test) and tended to become smaller for longer pulses, possibly due to accumulating channel inactivation and/or increasing depolarization-block of SGNs upon prolonged photo-depolarization (Figure 2.2H). In summary, the oABR comparison indicates greater temporal fidelity but higher required radiant flux for both vf-Chrimson-ES/TS- and vfChimson-mediated SGN stimulation than previously found for f-Chrimson (Mager et al, 2018). This is in line with the lower current density and the shorter deactivation time constant of vf-Chrimson expressed in NG cells (Mager et al, 2018).

Ultrafast optogenetic stimulation of the auditory pathway: recordings from single putative SGNS

To further evaluate the temporal fidelity of red-light optogenetic SGN stimulation mediate by vf-Chrimson-ES/TS and vf-Chrimson, we performed juxtacellular recordings from putative 
auditory nerve fibers (central axon of SGN) as previously described (Mager et al, 2018; Keppeler et al, 2018). As illustrated by the exemplary recordings in Figure 2.3, putative SGNs fired upon optogenetic stimulation with high temporal precision for stimulus rates of up to few hundreds of $\mathrm{Hz}$ without an obvious advantage of vf-Chrimson-ES/TS over vf-Chrimson. The temporal precision of firing, evaluated either with the vector strength (Figure 2.4A, i.e. spike synchronization in one period of stimulation, see methods for definition) or spike jitter (i.e., standard deviation of spike latency across pulses, Figure 2.4B), was generally high up to $100-200 \mathrm{~Hz}$ of stimulation. Temporal precision and spike probability (Figure 2.4C) diminished with increasing stimulation rates in both vf-Chrimson- and vf-Chrimson-ES/TSexpressing putative SGNs in a similar manner.

Spike jitter, calculated for spikes occurring in a time window comprised between two pulse onsets, increased with rate but was typically below a millisecond for stimulus rates lower than $300 \mathrm{~Hz}$ (Figure 2.4B). At higher stimulus rates, spike jitter increased beyond the values obtained for simulated Poisson spike trains (see methods, Figure 2.4C), indicating spike synchronization with the light pulses became less reliable. As a second temporal property, we calculated the spike latencies (Figure 2.4D) within the same time window, which remained fairly stable up to $300 \mathrm{~Hz}$. For a reference we replot the data from f-Chrimson-expressing putative SGNs recorded in our previous study (Mager et al, 2018). The comparison indicates comparable temporal fidelity of optogenetic SGN stimulation by vf-Chimson and f-Chrimson at least for stimulation rates up to $250 \mathrm{~Hz}$. We did not find a major advantage of addition of the ES/TS sequences for vf-Chrimson-mediated cochlear optogenetics, at least when using AAV-PHP.eB as the viral vector, the human synapsin promoter and by injecting the cochlea at P6. 
A
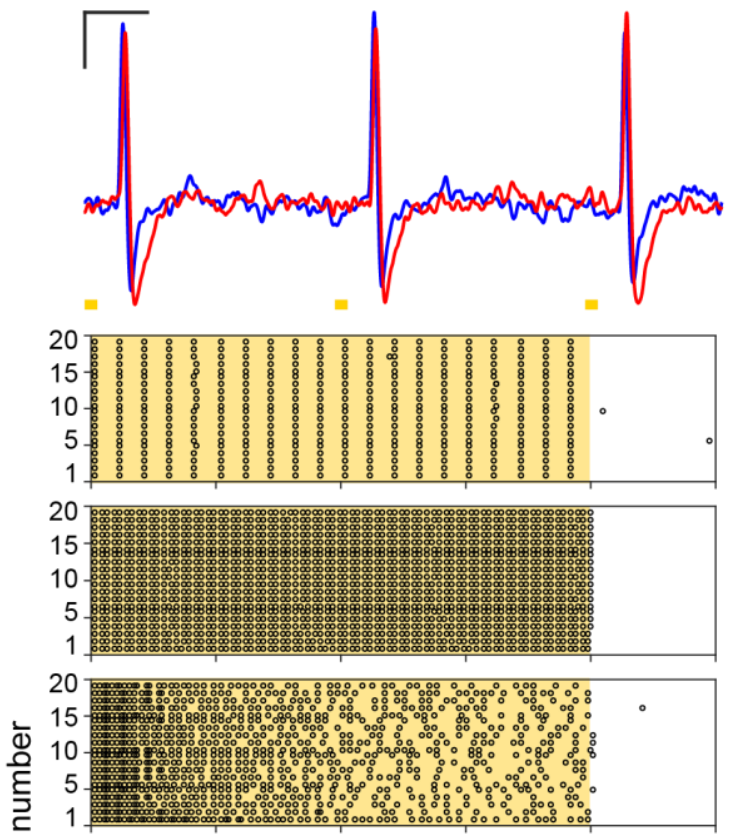

든 20

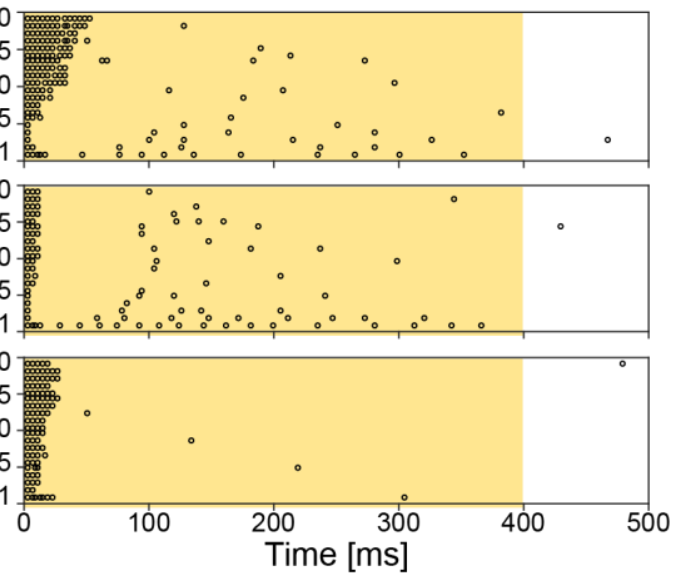

B

$1^{\text {st }}$ iteration

$20^{\text {th }}$ iteration

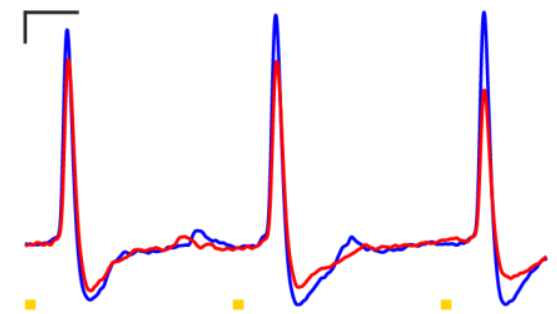

Stimulus rate
$50 \mathrm{~Hz}$
Pulse duration
$1 \mathrm{~ms}$

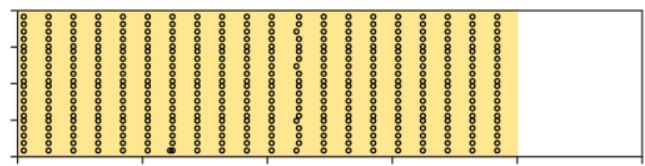

$200 \mathrm{~Hz}$

$1 \mathrm{~ms}$

$300 \mathrm{~Hz}$

$1 \mathrm{~ms}$

$500 \mathrm{~Hz}$

$1 \mathrm{~ms}$

$800 \mathrm{~Hz}$

$1 \mathrm{~ms}$

$1000 \mathrm{~Hz}$

$0.5 \mathrm{~ms}$
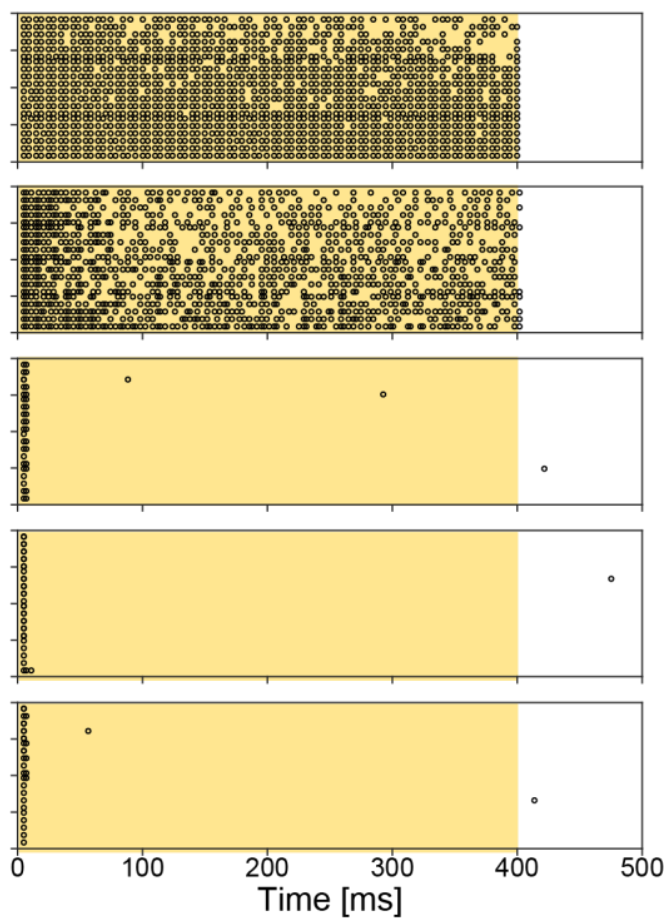

Figure 2.3. Characterizing optogenetic stimulation by extracellular recordings from single putative SGNs

(A, B) Exemplary spike traces at $50 \mathrm{~Hz}, 1 \mathrm{~ms}$, maximum radiant flux and corresponding raster plots at varying stimulation rates of vf-Chrimson- (A) and vf-Chrimson-ES/TS- (B) expressing putative SGNs. Raster plots showing spiking activity of the above units in response to $400 \mathrm{~ms}-$ long trains of laser pulses (shaded areas, at $43 \mathrm{~mW}, 1 \mathrm{~ms}$ for $20-800 \mathrm{~Hz}$, $500 \mu \mathrm{s}$ for $\geq 900 \mathrm{~Hz}$ ) recorded at six different stimulation rates over 20 iterations. Scale bars $0.1 \mathrm{mV}, 5 \mathrm{~ms}(\mathrm{~A})$ and $0.5 \mathrm{mV}, 5 \mathrm{~ms}(\mathrm{~B})$. 
A

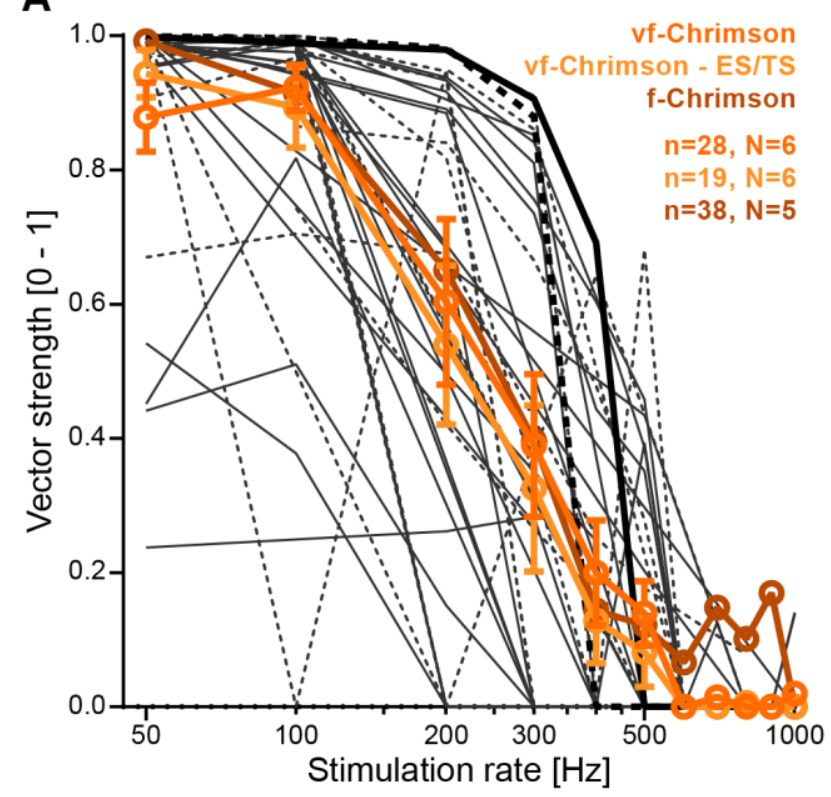

C

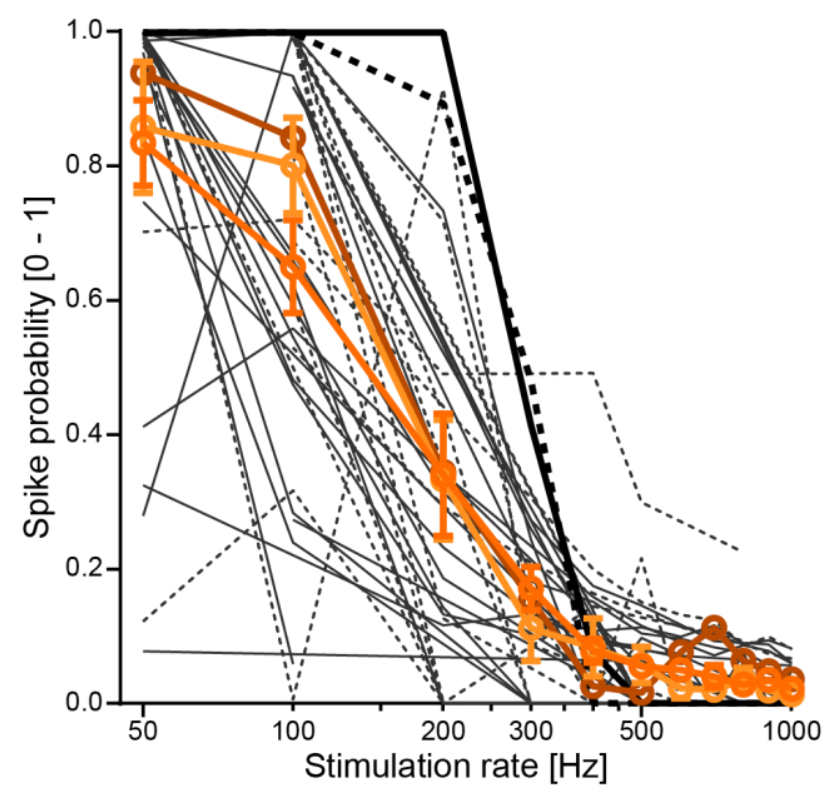

B

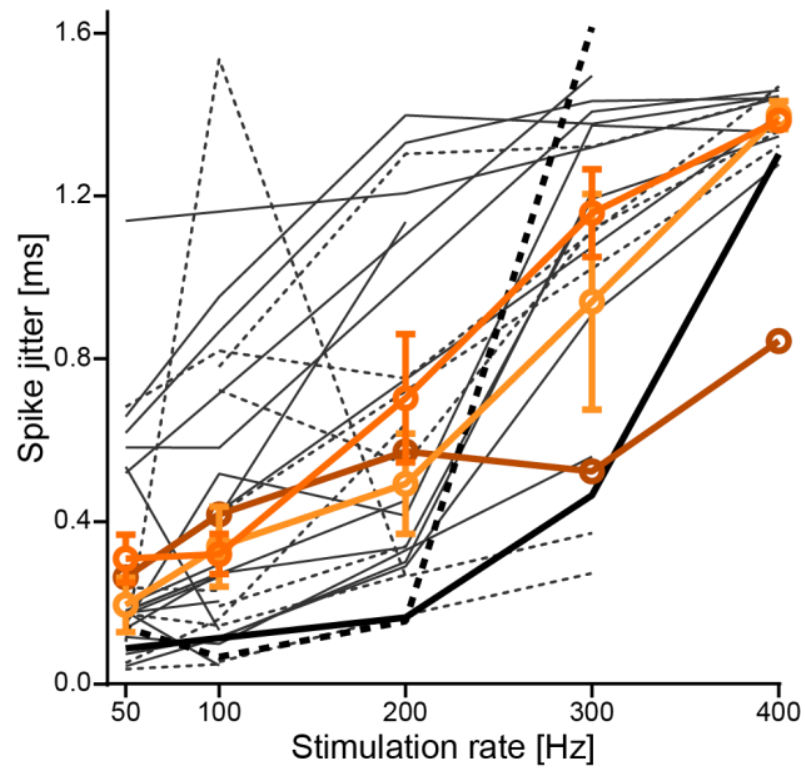

D

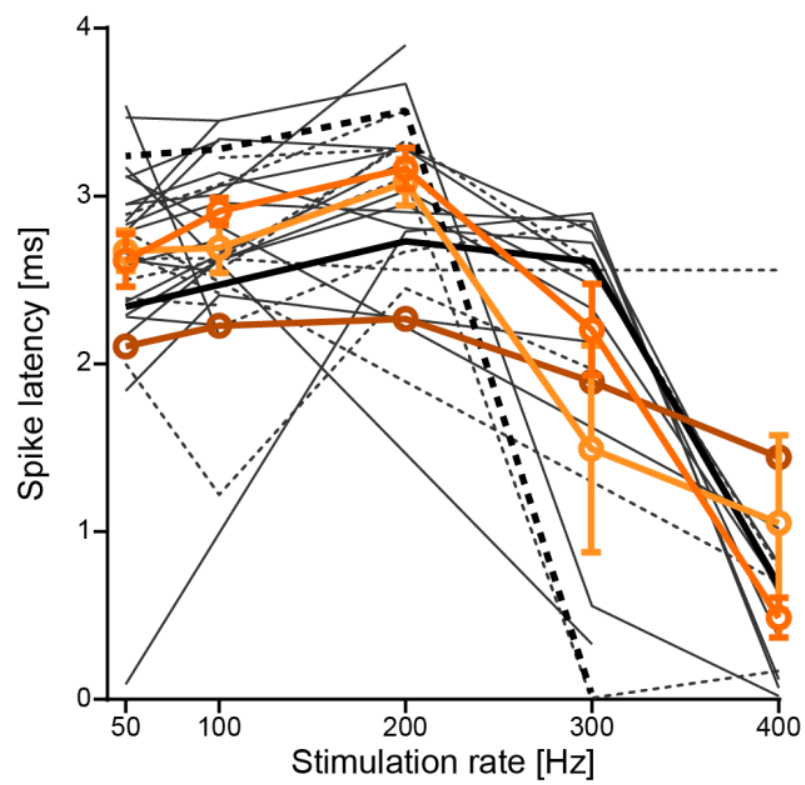

Figure 2.4. Analysis of temporal fidelity of optogenetic SGN stimulation by vf-Chrimson (A-D) Quantification of the vector strength (A), spike jitter (B), spike probability (C) and spike latency (D) as a function of repetition rate of putative SGNs. Colored points and lines show mean \pm SEM values for corresponding Chrimson construct, individual units are shown in gray only for vf-Chrimson (full lines) and vf-Chrimson-ES/TS (dashed lines). Average vector strength, first spike jitter, spike probability and first spike latency of f-Chrimson putative SGNs are replotted (Mager et al., 2018) in brown (note that only 7-5 putative SGNs showed responses beyond a first spike above $500 \mathrm{~Hz}$ of stimulation for f-Chrimson). The putative SGNs represented on Figure 2.3 are shown in black full (vf-Chrimson) and dashed lines (vf-Chrimson-ES/TS), respectively.

\section{Intensity coding by red ultrafast optogenetic stimulation}

Next, we studied the rate of optogenetically driven firing of putative SGNs as a function of light intensity to determine the output dynamic range of optogenetic coding at the single SGN 
level. Single SGNs are able to encode changes in sound intensity of about 5-50 dB SPL as measured in response to pure tones (Sachs \& Abbas, 1974; Liberman, 1978; Winter et al, 1990; Taberner \& Liberman, 2005; Huet et al, 2016). However, the full range of sound intensities audible is much larger, e.g. spanning 6 orders of magnitude or $120 \mathrm{~dB}$ in humans (Viemeister \& Bacon, 1988). This discrepancy is known as the "dynamic range problem" (Evans, 1981). To achieve wide dynamic range coding at the SGN population level, at least to some degree, the individual SGNs of one tonotopic place contribute in a complementary way to cover the full range of audible sound pressure level. Furthermore, in natural hearing, increments in sound pressure level correlate with the number of recruited SGNs (Furman et al, 2013; Bourien et al, 2014). Finally, neural adaptation is thought to contribute to wide dynamic range sound coding (Wen et al, 2012). In electrical hearing the dynamic range at the single SGN level is very limited ( 1 dB (Miller et al, 2006)) and the extensive spread of excitation leads to massive recruitment of neighboring SGNs even for low to modest stimulus intensities. Hence intensity coding by either mechanism is relatively poor with the eCI, which likely contributes to the limited speech perception in noise (Weiss et al, 2016; Zeng \& Galvin, 1999).

How individual ChR-expressing SGNs respond to changes in light intensity has not yet been studied. However, en route to developing optogenetic hearing restoration, it is of great importance to probe over what range of intensities SGN firing can be changed. Larger dynamic range of the response of single SGNs and higher spectral resolution would enable optogenetic SGN stimulation to improve intensity coding via both mechanisms of loudness coding. Here, we placed emphasis of the intensity coding analysis on f-Chrimson due to its excellent membrane expression, that causes low light energy requirements even following transduction with less potent AAVs (AAV2/6). In order to prepare the analysis of lightintensity coding by f-Chrimson-expressing SGNs (Figure EV2.1), we first compared the responses of putative SGNs to trains (range from 20 to $300 \mathrm{~Hz}, 400 \mathrm{~ms}$ duration, $100 \mathrm{~ms}$ recovery, 20 iterations) of saturating acoustic square clicks (300 $\mu \mathrm{s}$ width, $100 \mathrm{~dB}$ ) and optogenetic stimuli (pulse length of $1 \mathrm{~ms}, 18.3 \mathrm{~mW}$ ) finding peak firing probability at frequencies $20-100 \mathrm{~Hz}$, outperforming that of acoustical stimulation at $50 \mathrm{~Hz}(p=0.0027$; Mann-Whitney-U test). Firing probability declines as a function of stimulation frequency, which was more pronounced for the optogenetic response than for acoustic clicks (at 200 and $300 \mathrm{~Hz}, p<0.0001 ;$ Mann-Whitney-U test). Responses to light pulses and clicks were comparable at a stimulation rate of $100 \mathrm{~Hz}$, which was therefore chosen to evaluate SGN responses to changing stimulus intensity. 


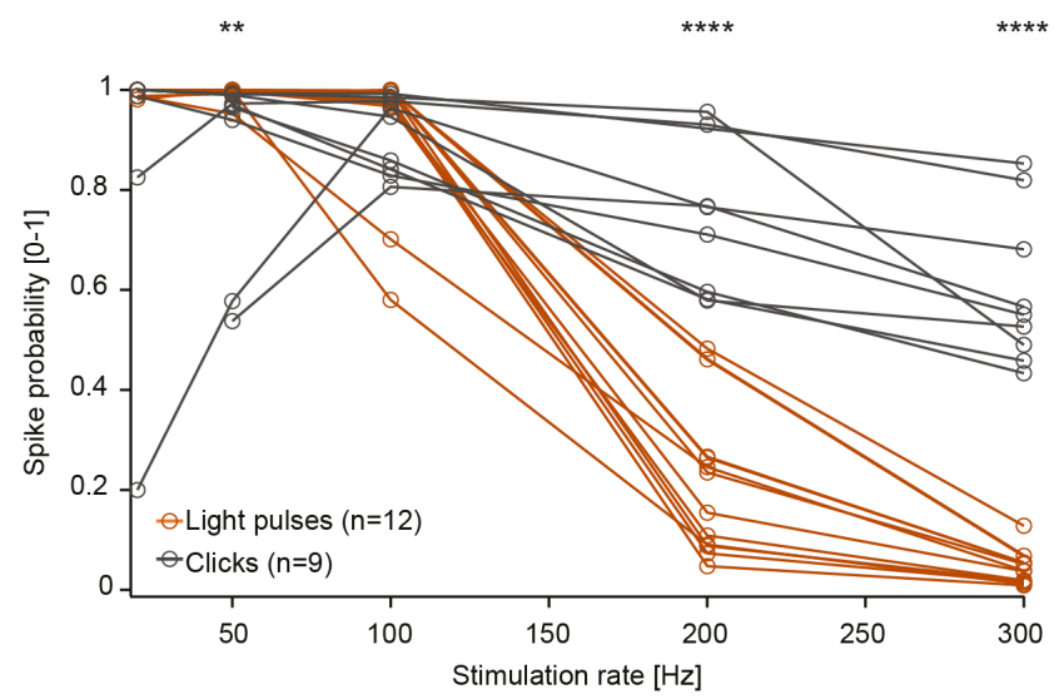

Figure EV2.1: Spike probability decreases with increasing rates of optogenetic and acoustic stimulation

Probability of firing for putative SGNs expressing f-Chrimson across different stimulation rates per acoustic click (grey, $100 \mathrm{~dB}$ SPL (pe), $300 \mu$ s click width at $100 \mathrm{~Hz} ; \mathrm{n}=12,3$ mice) and light pulse (orange, $18.3 \mathrm{~mW}, 1 \mathrm{~ms}$ pulse length at $100 \mathrm{~Hz} ; \mathrm{n}=9 ; 3$ mice) presented in a train over $400 \mathrm{~ms}$ leaving a recovery inter-train interval of $100 \mathrm{~ms}$ across repetitions per tested rate. Asterisks mark significant differences between stimulation modalities.

For a first characterization of optogenetic SGN responses to trains of light pulses of varying radiant flux, we calculated spike probability (mean number of spikes per pulse) across different stimulus intensities. At maximum radiant flux $(18.3 \mathrm{~mW})$, SGNs tended to fire 0.98 \pm 0.06 spikes per pulse while acoustic clicks elicited $1.26 \pm 0.14$ spikes per pulse at maximum sound pressure levels (100 dB (pe)) (Figure EV2.2A, B). To directly compare these two stimulus modalities, i.e. sound versus light, we present light radiant flux values in terms of decibels with respect to oABR activation threshold of f-Chrimson $(0.5 \mathrm{~mW})$ using the formula Intensity $_{\mathrm{dB}}(\mathrm{mW})=10 \times \log _{10}\left(\frac{\text { Intensity }(\mathrm{mW})}{\text { Threshold intensity }(\mathrm{mW})}\right)$. Next, we addressed the adaptation within the train as the ratio between the firing rate during the first $100 \mathrm{~ms}$ and the firing rate during the entire length of the train $(400 \mathrm{~ms})$. Interestingly, at low laser intensities, the adaptation ratio is higher for optogenetic stimulation, while for higher intensities it approaches $\sim 1$ (Figure EV2.2C, D). For acoustic stimulation, the adaptation ratio is maintained steady $(\sim 1)$ across sound pressure levels. At sound intensity levels of 40-50 dB SPL (pe [peak equivalent]), acoustically-driven SGNs show an adaptation ratio of $1.08 \pm 0.32$, significantly lower than that of $1.65 \pm 0.17$ for optically-stimulated SGNs at intensities of 2$4.5 \mathrm{~dB}(\mathrm{~mW})$ relative to $0.5 \mathrm{~mW}(p=0.0149$; Mann-Whitney-U test). 
A

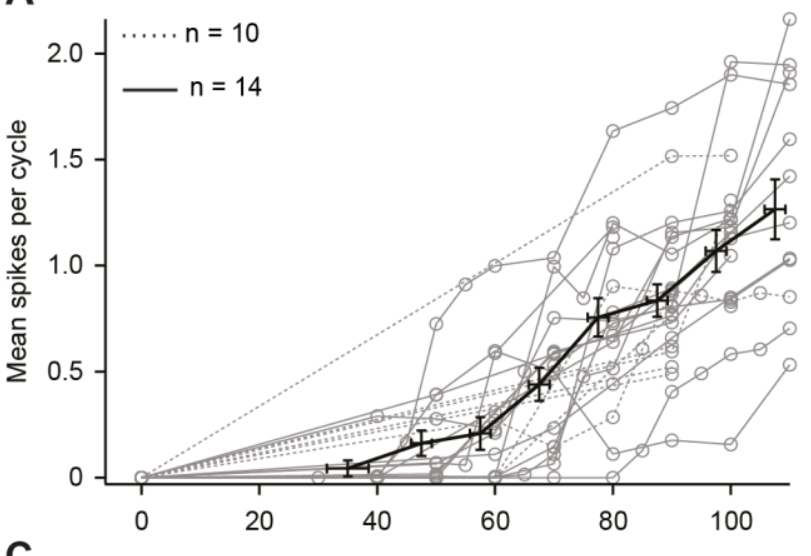

C

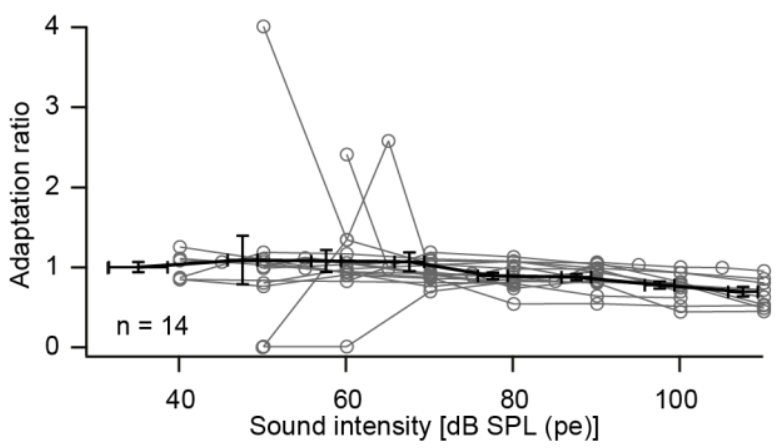

B
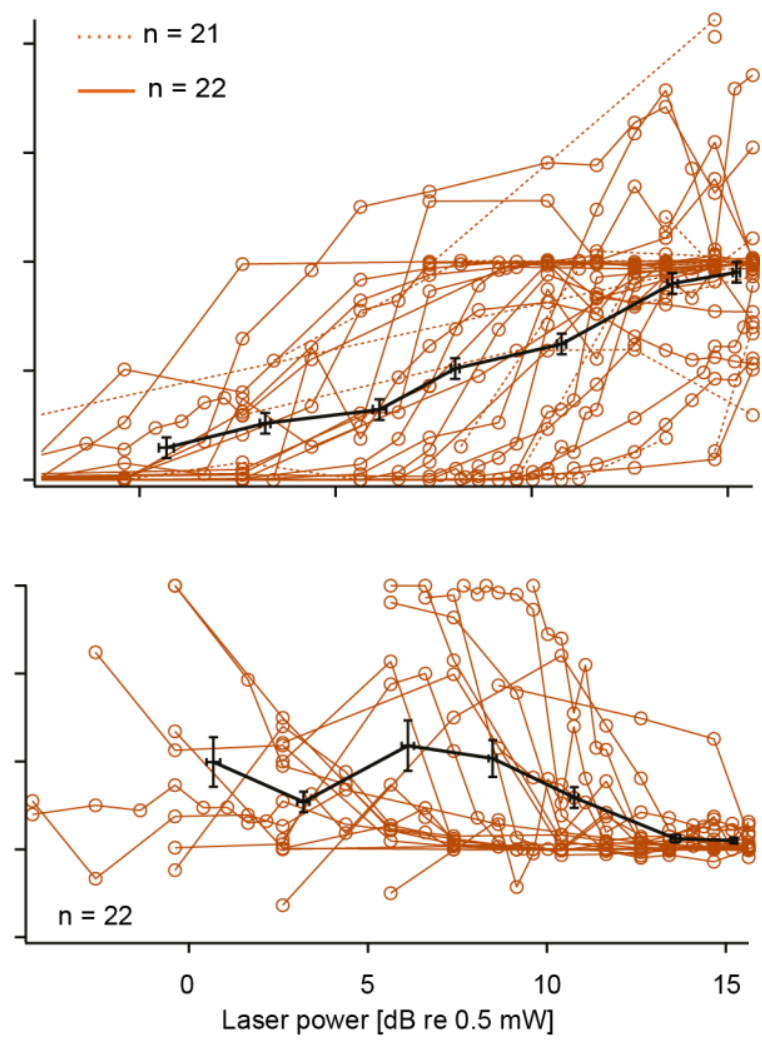

Figure EV2.2: High light intensities trigger mainly one spike per pulse while low light intensities mainly elicit spikes at the beginning of the stimulation train

(A, B) Mean number of spikes elicited per stimulation cycle in response to click stimulation (A) at increasing intensities ( $\mathrm{n}=24$ from 4 mice) and to optogenetic stimulation (B) $(n=33$ from 3 mice). Dashed lines in A and B show fibers discarded for RLF analysis due to insufficient data points (clicks: $\mathrm{n}=10$ units from 4 mice; light-pulses: $\mathrm{n}=16$ units from 3 mice). Solid lines represent responses from fibers for which RLF was further analyzed in Figure 2.5 (clicks: $n=14$ units from 4 mice; light-pulses: $n=17$ units from 3 mice). (C, D) Adaptation ratio (mean firing rate during the first $100 \mathrm{~ms}$ over mean firing rate along the entire stimulation train) of SGN responses to acoustic clicks (C) at increasing stimulus intensities $(n=14)$ and to light pulse trains (D) at increasing intensities $(n=17)$. Means $( \pm$ s.e.m.) are shown as black lines.

The relation of firing rate and stimulus intensity ("rate-level function", RLF) was constructed in separate experiments for $100 \mathrm{~Hz}$ trains of acoustic clicks and light pulses (Figure 2.5). fChrimson-expressing SGNs showed an average threshold of $5.38 \pm 1.13 \mathrm{~dB}(\mathrm{~mW})$ relative to $0.5 \mathrm{~mW}$ (previously calculated for oABR in response to $1 \mathrm{~ms}$ light pulses delivered at $10 \mathrm{~Hz}$, (Mager et al, 2018) while acoustically-stimulated SGNs had a mean threshold of $58.56 \pm 3.58$ dB SPL (pe). The mean threshold of the three most sensitive SGNs found in optical experiments was $-1.25 \pm 0.64 \mathrm{~dB}(\mathrm{~mW})$ relative to $0.5 \mathrm{~mW}$ per pulse, probably reflecting optimal f-Chrimson expression and illumination conditions. In both stimulation modalities, firing rates increased with stimulus intensity. 
For clicks, most RLFs grew with sound intensity showing "sloping saturation" (i.e. firing rate increased with sound intensity but did not saturate completely (Sachs \& Abbas, 1974). In contrast, firing of most light-stimulated SGNs showed "flat saturation" (Sachs \& Abbas, 1974): most SGNs fired one spike per pulse at saturating light intensities (27 out of 33 ANFs, $81.82 \%)$ and the remaining 6 ANFs (18.18\%) responded with an average of $1.61 \pm 0.19$ spikes per pulse. RLF growth was strongly monotonic for both stimulation modalities with higher monotonicity for clicks than for light pulses (median monotonicity index: $1.00 \mathrm{vs}$ 0.996; $p=0.0142$; Mann-Whitney-U test Figure 2.5E). RLFs grew with a steeper slope for optogenetic stimulation than for clicks: the maximal steepness amounted to $35.22 \mathrm{sp} / \mathrm{s} \times \mathrm{dB}^{-1}$ $\left(\mathrm{mW}\right.$ ) of optical stimulation compared to $5.49 \mathrm{sp} / \mathrm{s} \times \mathrm{dB}^{-1}$ SPL (pe) of acoustic clicks ( $p<$ 0.0001; Mann-Whitney-U test; Figure 2.5F). The dynamic range was estimated from sigmoidal fits to the RLFs and defined as the difference of the stimulus levels eliciting $10 \%$ and $90 \%$ of the difference between spontaneous and maximal driven firing rate. As expected from the steeper RLF, the dynamic range of optogenetic stimulation was smaller than for acoustical clicks $(3.87 \pm 0.47 \mathrm{~dB}(\mathrm{~mW})$ vs $31.29 \pm 4.46 \mathrm{~dB}$ SPL (pe), respectively; $p<$ 0.0001 , t-test; Figure 2.5G). 
A

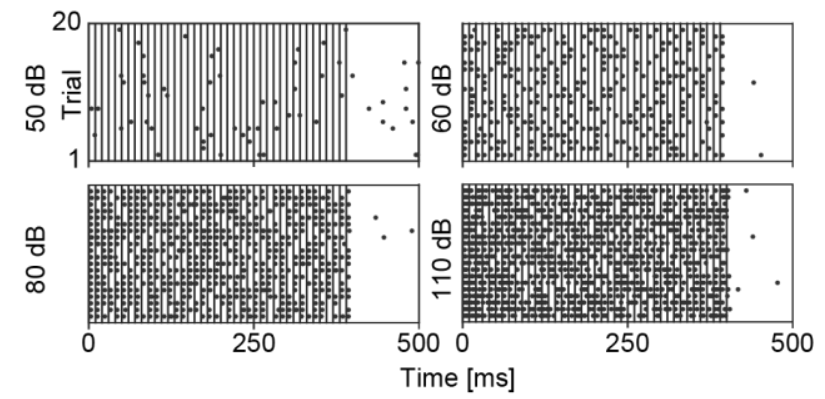

C

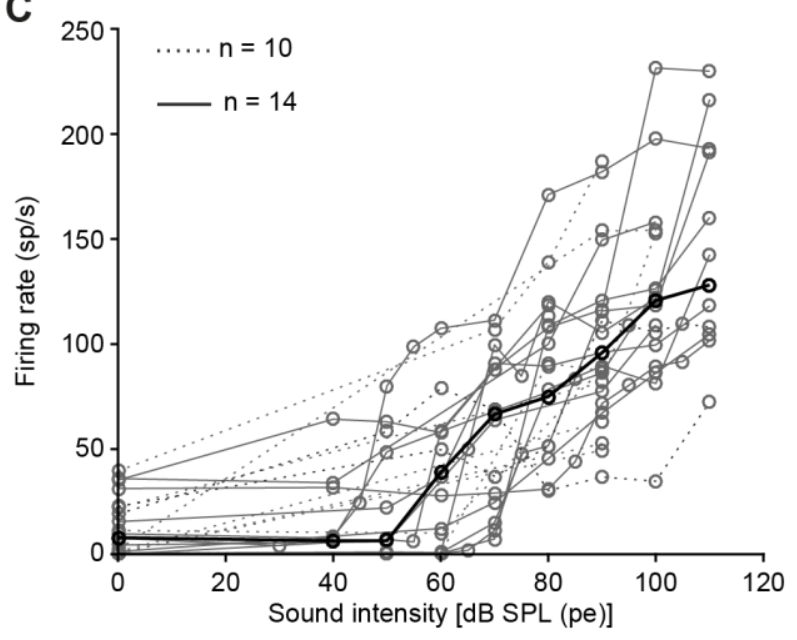

B

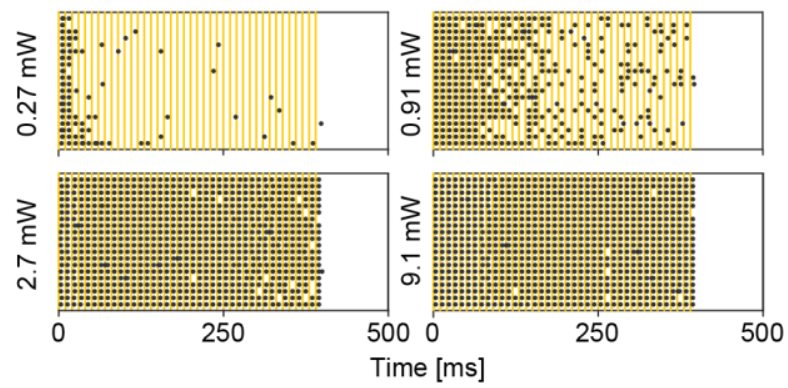

D

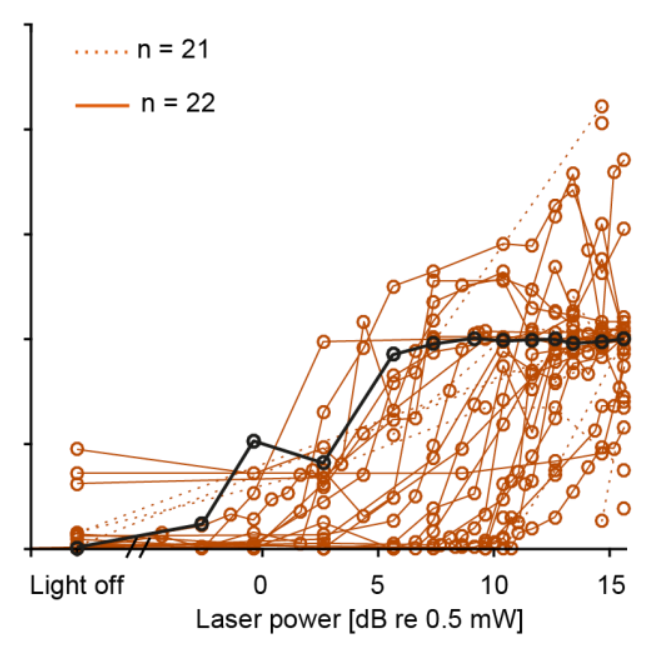

$E$

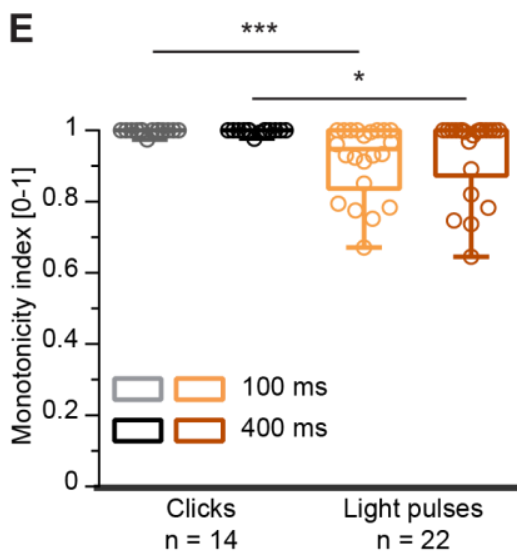

$\mathbf{F}$

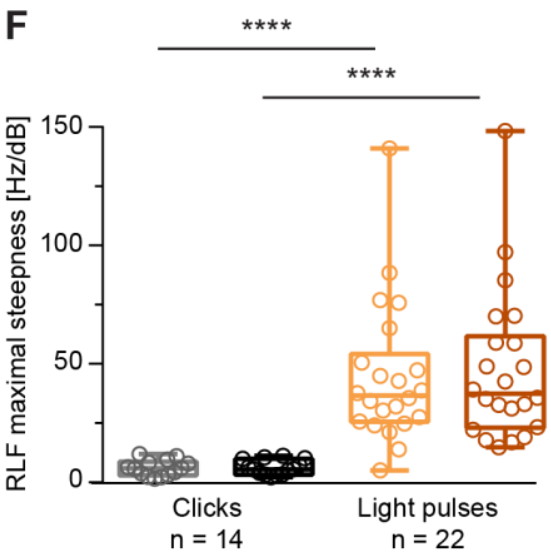

G
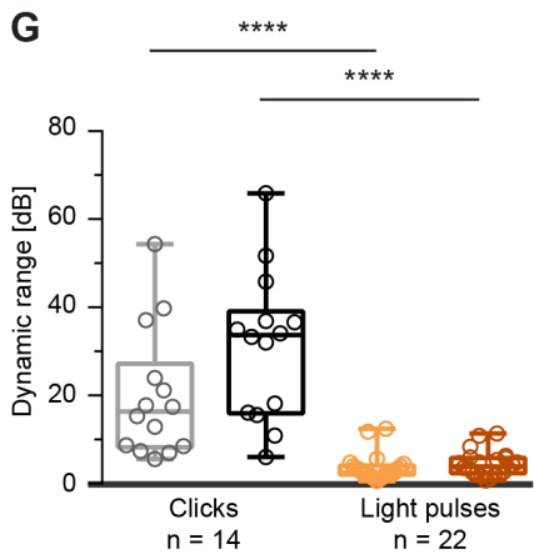

Figure 2.5: Rate-level functions of putative SGNs in response to clicks and light pulses

(A, B) Activity of exemplary putative SGNs in response to $400 \mathrm{~ms}$ long $100 \mathrm{~Hz}$ trains of 300 $\mu$ s clicks at different sound intensities (A) and of $1 \mathrm{~ms}, 594 \mathrm{~nm}$ light pulses at different radiant flux (B). (C, D) RLFs in response to acoustic click trains (C) for 24 single units from 4 mice and to light pulses (D) displayed for 33 single units from 3 mice. Dashed lines in C and D show RLFs discarded for further analysis (clicks: $n=10$ units from 4 mice; light-pulses: $n=$ 16 units from 3 mice). Solid lines represent RLFs that were further analysis shown in (E-G) (clicks: $\mathrm{n}=14$ units from 4 mice; light-pulses: $\mathrm{n}=17$ units from 3 mice). Black traces in $\mathrm{C}$ and D show data for units in A and B respectively. (E) Lower monotonicity of the firing response to optical stimulation (light orange: analysis of first $100 \mathrm{~ms}$ of train and orange: analysis of entire $400 \mathrm{~ms}$ train) than for clicks (grey: analysis of first $100 \mathrm{~ms}$ of train and black: analysis of entire $400 \mathrm{~ms}$ train). (F) maximal steepness of RLF is greater for optogenetic stimulation (color code as in E). (G) dynamic range estimated from the RLFs (range of stimulus intensity levels eliciting 10 and $90 \%$ of the difference between spontaneous 
and maximal driven rate) in acoustical (dB SPL (pe)) and optogenetic stimulation $(\mathrm{dB}(\mathrm{mW}))$, (color code as in E).

The stimulus intensity required for eliciting the half-maximal response (50\% of the difference between the spontaneous and maximal firing rate was on average $7.77 \pm 0.97 \mathrm{~dB}(\mathrm{~mW})$ relative to $0.5 \mathrm{~mW}$ ) for optogenetic stimulation, while for acoustical stimulation experiments it reached $75.91 \pm 2.90 \mathrm{~dB}$ SPL (pe) (Figure 2.5C). As mentioned, the SGNs with the diverse RLFs are thought to complement each other hence providing the brain with information covering a broader range of stimulation levels than conveyed by the individual SGN. To estimate the operating range of the population of optogenetically driven SGNs, we selected the three most responsive and the three least responsive units and calculated the difference in laser power between them at $50 \%$ of activation which amounted to $8.33 \pm 0.16 \mathrm{~dB}(\mathrm{~mW})$, wider than the average dynamic range of individual optogenetically driven SGNs.

\section{Temporal properties of SGN coding as a function of stimulus intensity}

Next, we analyzed the temporal properties of SGN firing in response to $100 \mathrm{~Hz}$ light/click pulse trains of f-Chrimson-expressing/naïve SGNs at different stimulus levels. The latency to the first spike elicited by a light pulse or click tended to decline with increasing stimulus intensity. At maximum stimulus levels, the mean first spike latency was significantly shorter for light-stimulated SGNs (2.59 $\pm 0.08 \mathrm{~ms}$ for f-Chrimson, $\mathrm{n}=33$ units from 3 mice) than in response to clicks $(2.90 \pm 0.22 \mathrm{~ms}, \mathrm{n}=24$ units from 4 mice, $p=0.0047$; Mann-Whitney-U test, Figure 2.6A,B). The temporal precision of firing was analyzed by means of the spike jitter and vector strength At maximum stimulus intensities, spiking was more precise in optical stimulation, as assessed by spike jitter $(0.39 \pm 0.05 \mathrm{~ms}$ for f-Chrimson vs. $1.06 \pm 0.17$ ms for acoustic clicks, $p=0.0002$; Mann-Whitney-U test, Figure 2.6C,D). 


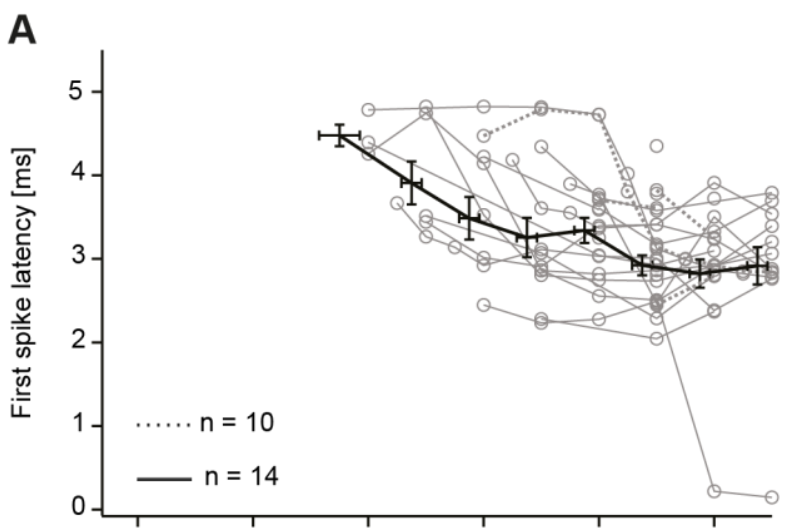

B
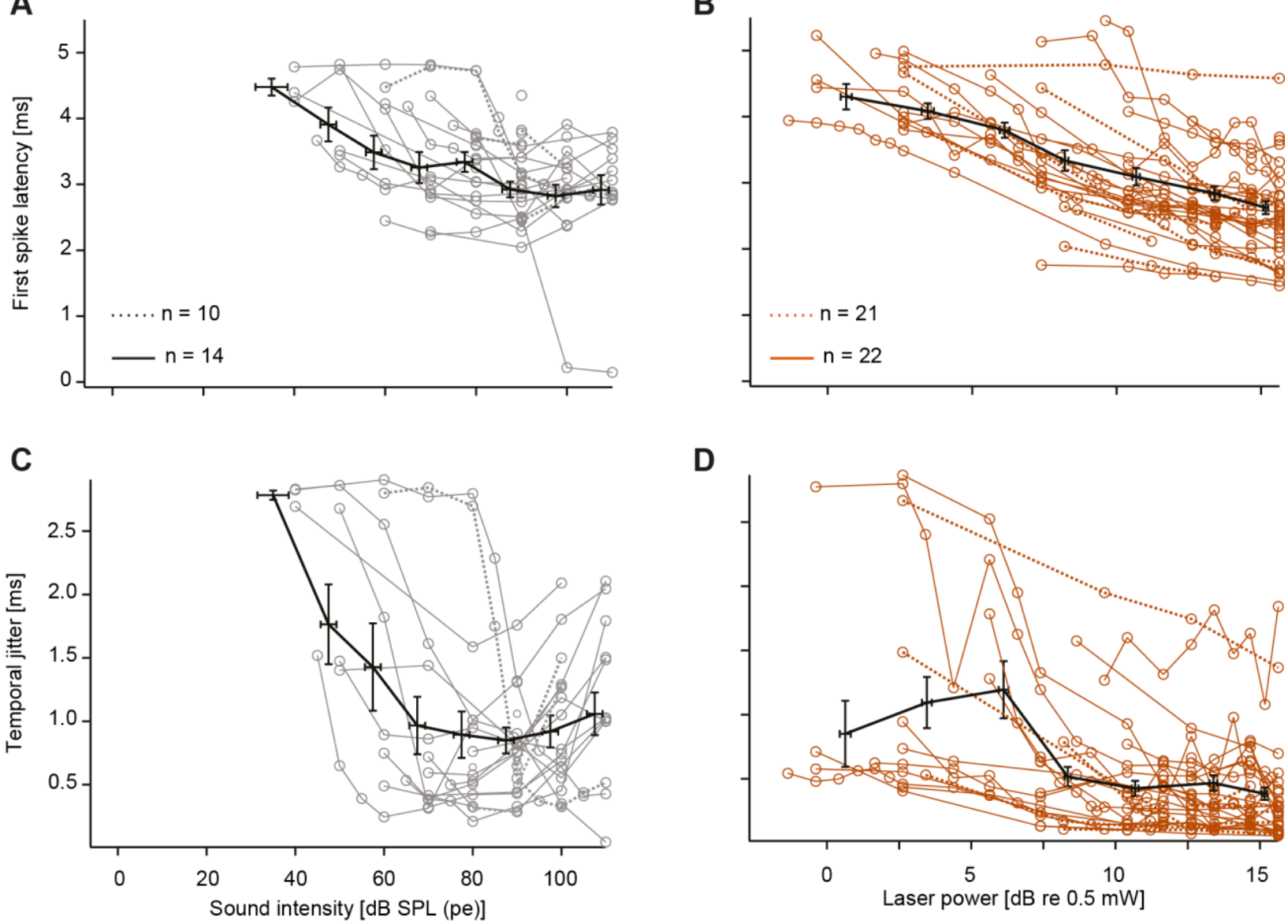

Figure 2.6: Temporal fidelity of firing as a function of stimulus intensity: probability and jitter

(A, B) Latency to the first spike per stimulation cycle elicited by clicks (A) of increasing intensity $(\mathrm{n}=24$ from 4 mice) and by light pulses $(\mathrm{B})(\mathrm{n}=33$ from 3 mice). Dashed lines in $\mathrm{A}$ and $B$ show fibers discarded for RLF analysis due to insufficient data points (clicks: $n=10$ units from 4 mice; light-pulses: $\mathrm{n}=16$ units from 3 mice; also applies to $\mathrm{C}$ and $\mathrm{D}$ ). Solid lines represent responses from fibers for which RLF was further analyzed in Figure 2.5 (clicks: $n=$ 14 units from 4 mice; light-pulses: $\mathrm{n}=17$ units from 3 mice; also applies to $\mathrm{C}$ and $\mathrm{D})$. (C, D) Jitter of the first elicited spike per stimulation cycle in acoustic experiments (C) and in optical stimulation ones (D). Means ( \pm s.e.m.) are shown as black lines.

For both stimulation modalities, vector strength increased steeply above its threshold (specific for each SGN). In response to acoustic clicks, once maximum phase locking level was reached, vector strength tended to decay at the highest intensities, while it was steadier once saturated in response to light pulses (medians: 0.98 for f-Chrimson, 0.84 for clicks, $p<$ 0.0001; Mann-Whitney-U test, Figure 2.7). 

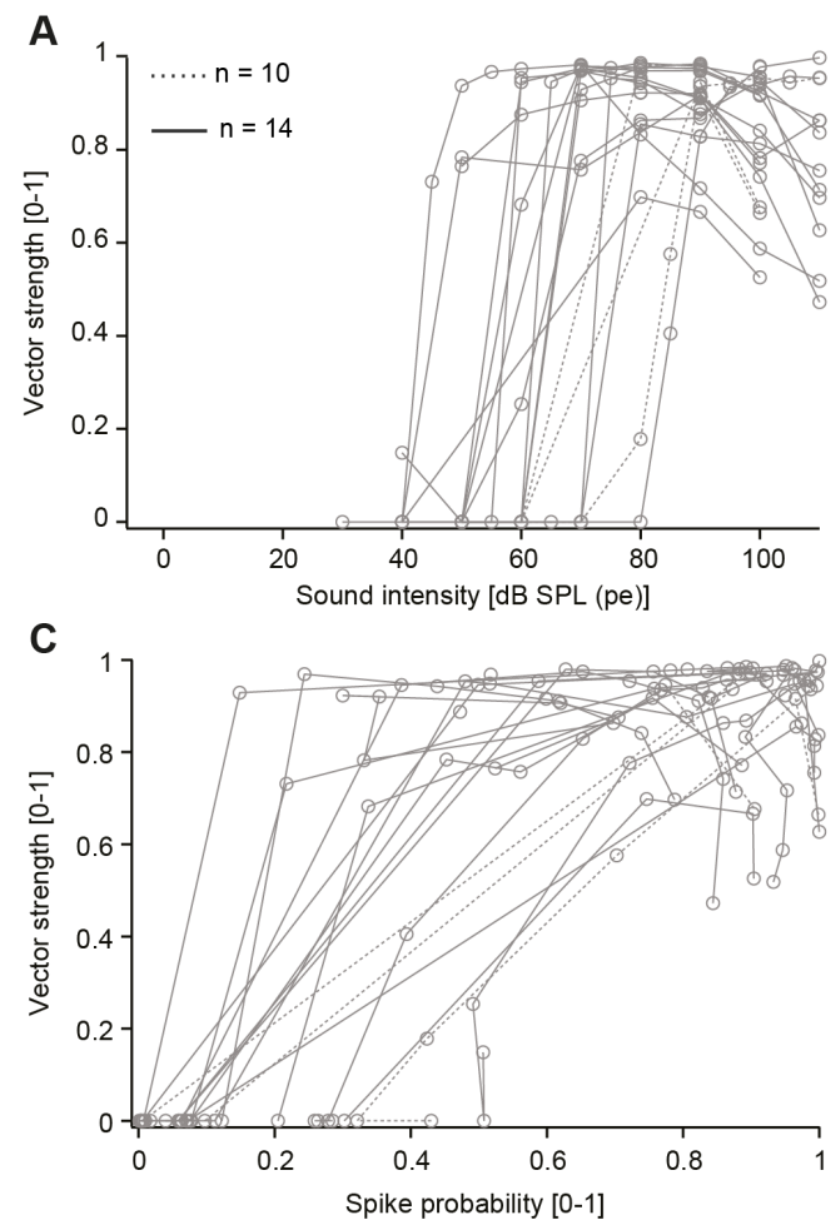

B

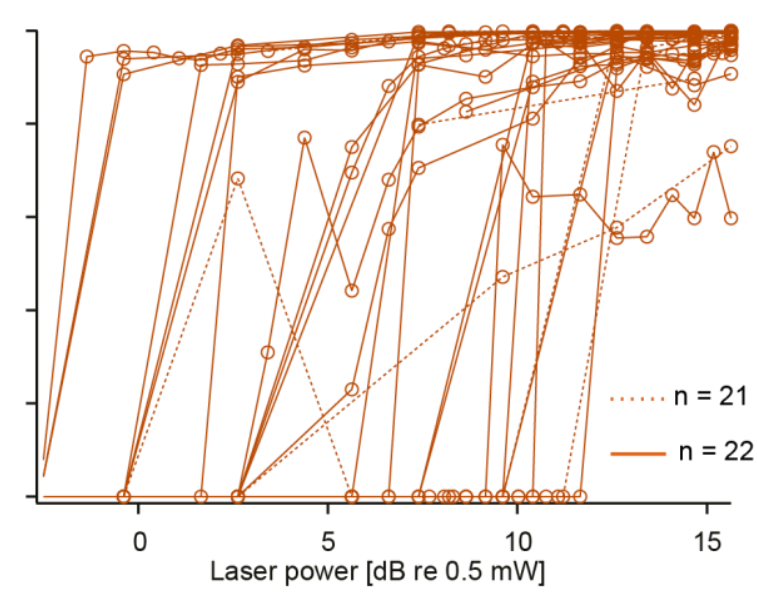

D

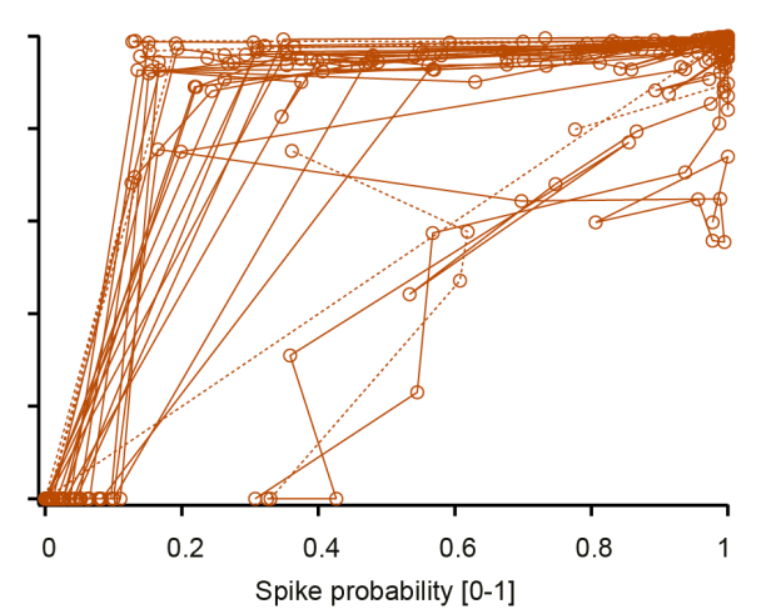

Figure 2.7: Temporal fidelity of firing as a function of stimulus intensity: vector stength (A, B) Vector strength of the SGNs response to acoustic click trains (A) of varying intensity $(n=24$ from 4 mice) and to light pulse trains $(B)(n=33)$. Dashed lines in A and B show fibers discarded for RLF analysis due to insufficient data points (clicks: $n=10$ units from 4 mice; light-pulses: $\mathrm{n}=16$ units from 3 mice; also applies to $\mathrm{C}$ and $\mathrm{D})$. Solid lines represent responses from fibers for which RLF was further analyzed in Figure 2.5 (clicks: $n=14$ units from 4 mice; light-pulses: $\mathrm{n}=17$ units from 3 mice; also applies to $\mathrm{C}$ and $\mathrm{D})$. (C, D) Variation of vector strength with spike probability in acoustically-stimulated fibers (C) and for optically-stimulated fibers (D). Lines in C and D connect data points obtained from the same SGNs. 


\section{Discussion}

Here, we characterized the optogenetic stimulation of SGNs mediated by the red-light activated ChRs f-Chrimson and vf-Chrimson. Following early postnatal injection of AAVs into the mouse cochlea, both ChRs were expressed very well by SGNs of the mouse cochlea. In contrast to previous findings with the very fast blue-light gated ChR Chronos, we did not find a major improvement of the utility of vf-Chrimson upon adding trafficking sequences derived from $\mathrm{K}^{+}$channel Kir2.1. Neural population responses of vf-Chrimson-expressing SGNs showed amplitudes above $200 \mathrm{nV}$ ( 10\% of maximal oABR amplitude) for stimulation rates $\geq 500 \mathrm{~Hz}$. On average, spike probability and phase locking quality of single vfChrimson-expressing SGNs were near $100 \%$ at $100 \mathrm{~Hz}$ and dropped to $50 \%$ around $200 \mathrm{~Hz}$ of stimulation for strong light pulses. This temporal fidelity of vf-Chrimson-mediated SGN coding is comparable to that of f-Chrimson- and Chronos-ES/TS-expressing SGNs. The dynamic range reported by oABR exceeded $13 \mathrm{~dB}(\mathrm{~mW})$ for f-Chrimson. For f-Chrimson, at the single SGNs level the dynamic range amounted to $3.9 \mathrm{~dB}(\mathrm{~mW})$, whereas at the population level extended to $8.33 \mathrm{~dB}(\mathrm{~mW})$. In conclusion, vf-Chrimson and f-Chrimson are valuable candidates for red-light optogenetic SGN stimulation.

\section{Ultrafast vf-Chrimson-mediated stimulation of the auditory pathway}

Stimulation of the auditory pathway is a prime example for an application of optogenetics requiring both high speed and light sensitivity. Upon sound stimulation in physiological hearing, SGNs fire at hundreds of $\mathrm{Hz}$ and show sub-millisecond temporal precision of spiking relative to the stimulus. Electrical stimulation achieves even higher temporal precision (Miller et al, 2006) such that eCIs typically employ very high stimulation rates ( $800 \mathrm{~Hz}$ and greater) to avoid overly synchronized activity in the auditory nerve that otherwise would be suspected to cause an unnatural hearing percept (Zeng, 2017). Here, we probed the utility of fast ChR with red-shifted action spectrum for stimulating SGNs firing. High temporal fidelity of optogenetically-driven SGN firing is a major objective for applications of the method in auditory research and future clinical hearing restoration. To a first approximation, one would expect the deactivation (closing) kinetics of the expressed $\mathrm{ChR}$ and radiant flux to govern the temporal fidelity of SGN firing. Consistent with this notion, the faster the closing kinetics of the ChR the higher the stimulation rate that the SGN population can respond to with a sizable compound action potential (20\% of maximal oABR $\mathrm{P}_{1}-\mathrm{N}_{1}$ amplitude): from $200 \mathrm{~Hz}$ for fChrimson with $\tau_{\text {off: }} 3 \mathrm{~ms}$ (Mager et al, 2018) to $500 \mathrm{~Hz}$ for vf-Chrimson ( $\tau_{\text {off: }} 1.6 \mathrm{~ms}$ (Mager et al, 2018)) to $1 \mathrm{kHz}$ Chronos with $\tau_{\text {off: }} 0.7 \mathrm{~ms}$ (Keppeler et al, 2018)). At the level of single 
putative SGNs, on average, 50\% spike probability and vector strength of 0.5 were observed at approximately $200 \mathrm{~Hz}$ of stimulation for f-Chrimson (Mager et al, 2018) and vf-Chrimson (this study) and 300-400 Hz for Chronos-ES/TS (Keppeler et al, 2018). We note that some putative SGNs showed good responses to even higher rates of stimulation (e.g. Figure 2.4 of this study).

Higher temporal fidelity of optogenetic coding comes at a price. The faster channel closing, i.e. the shorter the open channel lifetime, the higher the threshold for optical stimulation: the radiant flux threshold for oABR was found to be $6.6 \mathrm{~mW}$ for Chronos-ES/TS (Keppeler et al, 2018), $6.9 \mathrm{~mW}$ for vf-Chrimson-ES/TS (this study) and $0.5 \mathrm{~mW}$ for f-Chrimson (Mager et al, 2018). This consideration is highly relevant when it comes to chronic optogenetic stimulation such as in an optical CI. For example, coding of auditory signals using Chronos-mediated optogenetic SGN stimulation would require blue light doses risking phototoxic cellular damage and conflicting with regulations regarding light exposure (Calabuig et al, 2017; European Commission, 2006). However, membrane expression of the ChR is another important parameter, which likely explains at least part greater light sensitivity conveyed by fChrimson to SGNs than by vf-Chrimson, as f-Chrimson showed greater photocurrent densities at saturating irradiance also in NG cells. Efforts to optimize the membrane trafficking and residence of ChRs are critical for reducing the required light doses and the proteostatic stress of SGNs. These preclinical efforts should also aim for using trafficking sequences of human membrane proteins (such as Kir2.1) and evaluate the effect of omitting the fluorescent protein from the expression construct to enhance biosafety. While there was a tendency for lower oABR thresholds and greater oABR amplitudes for the traffickingoptimized vf-Chrimson-ES/TS, the optogenetic stimulation remained inferior to that mediated by f-Chrimson (e.g. Fig. 2). This might at least in part relate to the lower transduction rate observed in the present study upon early postnatal cochlear AAV-injection despite the use of likely more potent viral vectors (AAV-PHP.B and AAV-PHP.eB with transduction rates being statistically indistinguishable from each other) than used for f-Chrimson here and in (Mager et al, 2018), AAV6) with the same means of administration. More generally, we note that differences in titer and viral vector as well as trial to trial variability of the AAV-injection might contribute to the variance in the functional responses such as oABR.

Therefore, f-Chrimson was selected to further analyze the utility of optogenetic temporal coding and dynamic range in comparison to pulsatile acoustic stimulation using clicks. We found that the temporal precision of firing was significantly higher for optogenetic than for 
acoustic stimulation when driving SGNs at $100 \mathrm{~Hz}$ : at saturating stimulus levels, the temporal jitter was lower and the vector strength was slightly higher for optogenetic stimulation (Figs. 6 and 7). While interpretation of the latter needs to consider the fact that spontaneous firing was much less in the case of optogenetic experiments, we regard the lower jitter of evoked spikes to reflect the bypassing of the stochastic processes of sensory transduction and synaptic transmission in physiological coding. Temporal precision of coding increased with the level of stimulation for both acoustic and optogenetic coding. Interestingly, the vector strength and spike probabilities approached 1 already for low stimulus levels in the case of optogenetic stimulation, and remained high for higher stimulation levels. In contrast, for click stimulation vector strength tended to grow more gradually with spike probability and to decline with the highest levels. In conclusion, optogenetic stimulation with f-Chrimson provides near physiological temporal fidelity of coding with a favorable energy requirement and low risk of adverse radiation effects.

\section{Intensity coding SGNs expressing fast Chrimson}

Next to the utility of optogenetic stimulation for coding temporal (see above and (Keppeler et al, 2018; Mager et al, 2018) and spectral (Dieter et al, 2019; Hernandez et al, 2014) information, the range of stimulus intensities over which the firing rate of SGNs change is an important parameter to study. So far estimates of this so-called output dynamic range of the activation of the auditory pathway were based either on oABR amplitude (approximately 10$16 \mathrm{~dB}(\mathrm{~mW})$ ) (Mager et al, 2018; Wrobel et al, 2018) or multi-unit firing rate in the inferior colliculus (approximately $11 \mathrm{~dB}(\mathrm{~mW})$ (Dieter et al, 2019)). It is important to note here, that in all cases saturation of the response was not reached with the radiant fluxes used, hence these estimates represent an "apparent dynamic range" as a lower bound. Importantly, the range over which the individual SGN change their firing probability had not yet been investigated. Here, using f-Chrimson-mediated fiber-based optogenetic stimulation we found the output dynamic range at the single SGN level to amount to $\sim 4 \mathrm{~dB}(\mathrm{~mW})$ or $\sim 8 \mathrm{~dB}(\mu \mathrm{J})$. This presents a major advantage over the output dynamic range of electrical SGN stimulation ( $\sim 1 \mathrm{~dB}$ (current level), (Miller et al, 2006)) but still does not get close to our average estimate of SGN stimulation by acoustic clicks ( $\sim 31 \mathrm{~dB}$ (SPL). When deriving a population operating range from the sample of recorded f-Chrimson-expressing SGNs we obtained $\sim 8 \mathrm{~dB}(\mathrm{~mW})$ or $\sim 16 \mathrm{~dB}(\mu \mathrm{J})$. Obviously, the lifetime of an SGN recording did not allow us to grade the stimulus intensity in a very fine-grained manner as would be useful to derive physiological estimates of discernible intensity levels. This calls for behavioral experiments to provide intensity discrimination limens (King et al, 2016). 
Towards developing the optogenetic cochlear implant: evaluating the utility of optogenetic SGN stimulation in comparison to physiological and electrical stimulation

This study used recordings of population and single SGN activity to parametrize the utility of optogenetic SGN stimulation for coding time and intensity information. The obtained estimates provide important input for planning sound coding strategies for future optical CIs. It becomes clear, that coding strategies should target stimulation rates $<500 \mathrm{~Hz}$ for efficient yet sufficiently stochastic coding, rendering unnecessary the very high stimulation rates employed in eCIs. Indeed, studies of speech understanding as a function of stimulation rate in eCI users report no further gain beyond $500 \mathrm{~Hz}$ (Shannon et al, 2011). In order to maintain a reasonable battery lifetime, lowering the stimulation rate from the current state of art seems imperative: as the energy requirement per pulse of optogenetic stimulation (several $\mu \mathrm{J}$ ) currently exceeds that of the electrical cochlear implants (less than a $\mu \mathrm{J}$ (Zierhofer et al, 1995)), and since the goal is to significantly increase the number of stimulation channels given the greater frequency selectivity (Dieter et al, 2019). Clearly the light emitted from simultaneously activated stimulating channels will partially overlap and hence the energy budget will likely scale sublinearly with the number of channels in the oCI. Nonetheless, from the energetic point of view it seems that the apparent dynamic range found for optogenetic stimulation will be a realistic estimate also for a clinical implementation.

\section{Materials and Methods}

\section{Preparation of expression constructs}

We used pcDNA-vf-Chrimson-EYFP (generous gift from Ernst Bamberg) as starting material for the preparation of expression constructs. This construct was digested with BamHI/HindIII (NEB) and fragment containing vf-Chrimson-EYFP was gel extracted (Zymo Research) and further used for ligation. At the same time the plasmid pAAV_hSyn_f-Chrimson-EYFP (Mager et al., 2018) was also digested using restriction enzymes BamHI/HindIII and used as a backbone plasmid. This material was used to perform In-fusion cloning (TaKaRa/Clontech) and PCR reaction with the following primers: 5'AATTCAAGCTGCTAGCATGGCTGAGCTGATCAG-3' and 5'CCTGCTCTTGACCGGTCACTGTGTCCTCGT-3'. In the second step, the obtained PCR fragment was gel extracted (Zymo Research) and used for In-fusion ligation with the backbone plasmid pAAV_hSyn_Chronos-ES/TS (Keppeler et al., 2018) derived from digestion with the restriction enzymes NheI/AgeI (NEB). All obtained ligation products were further tested by the restriction enzyme digestion and finally sequenced externally. 


\section{Virus purification}

AAVs were generated in HEK-293T cells (ATCC) using polyethylenimine transfection (25.000 MW, Polysciences, USA) (Gray et al, 2011; Deverman et al, 2016). The cell line was regularly tested negatively for mycoplasma. In brief, triple transfection of HEK-293T cells was performed using the pHelper plasmid (TaKaRa/Clontech), the trans-plasmid providing viral capsid PHP.B (generous gift from Ben Deverman and Viviana Gradinaru, Caltech, USA) or PHP.eB (PHP.eB was a gift from Viviana Gradinaru (Addgene plasmid \# 103005; http://n2t.net/addgene:103005; RRID:Addgene_103005) and the cis-plasmid providing vfChrimson or vf-Chrimson-ES/TS (Figure 2.1A). We harvested viral particles $72 \mathrm{~h}$ after transfection from the medium and $120 \mathrm{~h}$ after transfection from cells and the medium. Viral particles from the medium were precipitated with $40 \%$ polyethylene glycol 8000 (Acros Organics, Germany) in $500 \mathrm{mM} \mathrm{NaCl}$ for $2 \mathrm{~h}$ at $4{ }^{\circ} \mathrm{C}$ and, after centrifugation at $4,000 \mathrm{~g}$ for $30 \mathrm{~min}$, combined with cell pellets for processing. The cell pellets were suspended in $500 \mathrm{mM}$ $\mathrm{NaCl}, 40 \mathrm{mM}$ Tris, $2.5 \mathrm{mM} \mathrm{MgCl} 2, \mathrm{pH} 8$, and $100 \mathrm{U} \mathrm{mL}^{-1}$ of salt-activated nuclease (Arcticzymes, USA) at $37^{\circ} \mathrm{C}$ for $30 \mathrm{~min}$. Afterwards, the cell lysates were centrifuged at 2,000 $\mathrm{g}$ for $10 \mathrm{~min}$ and AAVs purified over iodixanol (Optiprep, Axis Shield, Norway) step gradients $(15 \%, 25 \%, 40 \%$ and $60 \%$ ) (Zolotukhin et al, 1999; Grieger et al, 2006) at 58,400 rpm for $2.25 \mathrm{~h}$. AAVs were concentrated using Amicon filters (EMD, UFC910024) and formulated in sterile phosphate-buffered saline (PBS) supplemented with $0.001 \%$ Pluronic F-68 (Gibco, Germany). Virus titers were measured using an AAV titration kit (TaKaRa/Clontech) according to manufacturer's instructions by determining the number of DNase I resistant vg using qPCR (StepOne, Applied Biosystems). Purity of produced viruses was routinely checked by silver staining (Pierce, Germany) after gel electrophoresis (Novex ${ }^{\mathrm{TM}}$ 4-12 \% Tris-Glycine, Thermo Fisher Scientific) according to manufacturer's instruction. The presence of viral capsid proteins was positively confirmed in all virus preparations. Viral stocks were kept at $-80{ }^{\circ} \mathrm{C}$ until the injection.

\section{Postnatal AAV injection into the cochlea}

Postnatal AAV-injection into scala tympani of the left ear via the round window was performed at p6 wild-type C57BL/6 mice essentially as described in (Huet \& Rankovic, 2021) using AAV-PHP.B and AAV-PHP.eB viral capsids and hSyn promoter to drive transgenic expression of opsins in SGNs. In brief, under general isoflurane anaesthesia and local analgesia achieved by means of xylocaine, the left ear was approached via a dorsal incision and the round window membrane was identified and gently punctured using a borosilicate capillary pipette that was kept in place to inject approximately 1-1.5 $\mu 1$ of AAV2/6_hSyn- 
Chrimson (9.9E+12 genome copies/ml), PHP.eB_hSyn-vf-Chrimson-ES/TS $(1.1 \mathrm{E}+13$ genome copies/ml) or PHP. B_hSyn-vf-Chrimson -EYFP (8.7E+12 genome copies/ml). After virus application, the tissue above the injection site was repositioned and the wound was sutured and buprenorphine $\left(0.1 \mathrm{mg} \mathrm{kg}^{-1}\right)$ was applied as a pain reliever during surgery. Recovery of the animals was then tracked daily. In all experiments, mice were randomly selected for injection. No blinding was possible since injections have to be performed in the left ear leaving the right ear as an internal control. Hence, surgery prior to stimulation needed to be done in the injected ear. Animals were then kept in a $12 \mathrm{~h} \mathrm{light/dark}$ cycle, with access to food and water ad libitum. All experiments were done in compliance with the national animal care guidelines and were approved by the board for animal welfare of the University Medical Center Goettingen and the animal welfare office of the state of Lower Saxony (LAVES; 14/1726 and 17/2394). The calculation of animal number was performed prior to starting experiments employing the Wilcoxon Rank Sum Test and an error probability alpha smaller than 0.05 , a power (1-beta) of 0.95 and effect size depending on the precise experimental protocol.

\section{Immunostaining and imaging of cochlear cryosections}

Cochleae were fixed with $4 \%$ paraformaldehyde in phosphate buffered saline $(1 \mathrm{~h})$. Sections of the cochlea were cryosectioned following 0.12 M EDTA decalcification. After incubation of sections for $1 \mathrm{~h}$ in goat serum dilution buffer (16\% normal goat serum, $450 \mathrm{mM} \mathrm{NaCl}$, $0.6 \%$ Triton $\mathrm{X}-100,20 \mathrm{mM}$ phosphate buffer, $\mathrm{pH}$ 7.4) primary antibodies were applied over night at $4^{\circ} \mathrm{C}$. The following antibodies were used: chicken anti-GFP (catalog n.: ab13970, Abcam, 1:500), guinea pig anti-parvalbumin (catalog no.: 195004, Synaptic Systems, 1:300). Thereafter, secondary AlexaFluor-labeled antibodies (goat anti-chicken $488 \operatorname{IgG}(\mathrm{H}+\mathrm{L})$, catalog no.: A-11039, Thermo-Fisher Scientific, 1:200; goat-anti guinea pig $568 \operatorname{IgG}(\mathrm{H}+\mathrm{L})$, catalog no. A1107, Thermo-Fisher Scientific, 1:200) were applied for $1 \mathrm{~h}$ at room temperature. Confocal images were collected using a SP5 microscope (Leica) and processed in ImageJ. Expression was considered positive when anti-GFP immunofluorescence in a given cell (marked by anti-parvalbumin immunofluorescence) was found to be higher than $3 \mathrm{xSD}$ above the background fluorescence.

For analysis of ChR distribution line profiles (length: $7.5 \mu \mathrm{m}$, width: 3 pixels) were centered to the outer edge of the estimated SGN cell membrane. The line profiles were oriented perpendicular to the cell edge. For membrane/intracellular expression ratio a maximum peak 
detection was performed for membranous area (defined as $0 \mu \mathrm{m}$ ) and for intracellular area (defined as $1.12 \mu \mathrm{m}$ ).

\section{Animal surgery}

Mice were anesthetized with i.p. administration of a mixture of xylazine $\left(5 \mathrm{mg} \mathrm{kg}^{-1}\right)$ and urethane $\left(1.32 \mathrm{mgkg}^{-1}\right)$. Buprenorphine $\left(0.1 \mathrm{mg} \mathrm{kg}^{-1}\right)$ was used as an analgesic. Temperature was maintained constant at $37^{\circ} \mathrm{C}$ by means of a custom-designed heat plate ion a vibrationisolated table in a sound-proof chamber (IAC $\mathrm{GmbH}$, Niederkrüchten, Germany). For auditory nerve recordings, a tracheostomy was performed before the animals were positioned in a custom-designed stereotactic head holder. Pinnae were e, scalp reflected, portions of the lateral interparietal and of the left occipital bone removed, and a partial cerebellar aspiration performed to expose the surface of the cochlear nucleus.

\section{Optical stimulation in vivo}

The left bulla was reached using a retroauricular approach and opened to expose the cochlea. A $50 / 200 \mu \mathrm{m}$ optical fiber coupled to a $594 \mathrm{~nm}$ laser (OBIS LS OPSL, $100 \mathrm{~mW}$, Coherent Inc.) was inserted into the cochlea via the round window. Irradiance was calibrated with a laser power meter (LaserCheck; Coherent Inc.).

\section{Auditory brainstem responses}

For stimulus generation and presentation, data acquisition, and off-line analysis, we used a NI System and custom-written Matlab software (The MathWorks, Inc.). Optically-evoked ABRs (oABRs) and acoustically-evoked ABRs (aABRs) were recorded by needle electrodes underneath the pinna, on the vertex, and on the back near the legs. The difference potential between vertex and mastoid subdermal needles was amplified using a custom-designed amplifier, sampled at a rate of $50 \mathrm{kHz}$ for $20 \mathrm{~ms}$, filtered $(300-3000 \mathrm{~Hz})$ and averaged across 1000 presentations. The first ABR wave was detected semi-automatically with a customwritten Matlab script in which the wave was detected for each trace in a temporal window defined by the user. Thresholds were determined by visual inspection as the minimum sound or light intensity that elicited a reproducible response waveform in the recorded traces.

\section{Juxtacellular recordings from single putative SGNS}

For auditory nerve recordings, glass microelectrodes ( $50 \mathrm{M} \Omega)$ were advanced through the posterior end of the anteroventral cochlear nucleus using an Inchworm micro-positioner (EXFO Burleigh, NY, USA) and aimed towards the internal auditory canal. Action potentials were amplified using an ELC-03XS amplifier (NPI Electronic, Tamm, Germany), filtered 
(300-20000 Hz), digitized (National Instruments card PCIe-6323), analysed and prepared for display using custom-written Matlab (The MathWorks, Inc.) software. When light-responsive fibers were found, $400 \mathrm{~ms}$-long pulse trains at repetition rates $20-1000 \mathrm{~Hz}$ were presented, leaving $100 \mathrm{~ms}$ inter-train recovery over 20 iterations for each tested rate. Different rates were tested following no particular order, $20 \mathrm{~Hz}$ being the first repetition rate presented across all units. For repetition rates higher or equal to $200 \mathrm{~Hz}$, parameters were computed if the spike probability was equal to or greater than $5 \%$. If not, values were set to 0 . Phase-locking was quantified using the vector strength (Goldberg \& Brown, 1969), considering a cycle starting at the onset of a light pulse and ending at the onset of the subsequent pulse, and conforming to the equation: vector strength $=\frac{\sqrt{\left[? ?_{\mathrm{i}=1}^{\mathrm{n}} \cos ?_{\mathrm{i}}\right]^{2}+\left[?_{\mathrm{i}=1}^{\mathrm{n}} \sin ?_{\mathrm{i}}\right]^{2}}}{\mathrm{n}}, \Theta_{1}, \Theta_{2}, \ldots, \Theta_{n}$ cycle phases in which spikes occurred. The Rayleigh test was used to evaluate the significance of vector strength: if $\mathrm{L}>13.8$, the null hypothesis is rejected at the 0.001 significance level (Hillery \& Narins, 1987) and insignificant VS were set to 0 . The spike probability was calculated as the ratio between the number of spikes and the number of light-pulses. The temporal jitter is the standard deviation of spike latency across trials. The hazard function (for the temporal jitter analysis) was calculated for each stimulation rate by simulating spiking as a Poisson process at given rates (from 10 to 1000 spikes s${ }^{-1}$ ).

\section{Data analysis}

The data were analysed using Matlab (Mathworks), Excel (Microsoft), Igor Pro (Wavemetrics), FIJI (ImageJ2), Origin (Microcal Software), and GraphPad Prism (GraphPad Software). Averages were expressed as mean \pm SEM or mean $\pm \mathrm{SD}$, as specified in the captions. References to data in the main text were expressed as mean \pm SEM. For statistical comparison between two groups, data sets were tested for normal distribution (the D'Agostino \& Pearson omnibus normality test or the Shapiro-Wilk test) and equality of variances (F-test) followed by two-tailed unpaired Student's t-test, or the unpaired two-tailed Mann-Whitney U test when data were not normally distributed and/or variance was unequal between samples.

For evaluation of multiple groups, statistical significance was calculated by using one-way ANOVA test followed by Tukey's test for normally distributed data (equality of variances tested with the Brown-Forsythe test) or one-way Kruskal-Wallis test followed by Dunn's test for non-normally distributed data. 


\section{References and Notes}

Bourien J, Tang Y, Batrel C, Huet A, Lenoir M, Ladrech S, Desmadryl G, Nouvian R, Puel J-L \& Wang J (2014) Contribution of auditory nerve fibers to compound action potential of the auditory nerve. J. Neurophysiol. 112: 1025-1039

Busskamp V, Picaud S, Sahel JA \& Roska B (2012) Optogenetic therapy for retinitis pigmentosa. Gene Ther. 19: 169-175

Calabuig A, Mugnano M, Miccio L, Grilli S \& Ferraro P (2017) Investigating fibroblast cells under "safe" and "injurious" blue-light exposure by holographic microscopy. J. Biophotonics 10: 919-927

Chan KY, Jang MJ, Yoo BB, Greenbaum A, Ravi N, Wu W-L, Sánchez-Guardado L, Lois C, Mazmanian SK, Deverman BE \& Gradinaru V (2017) Engineered AAVs for efficient noninvasive gene delivery to the central and peripheral nervous systems. Nat. Neurosci. 20: 1172-1179

Commission E (2006) Directive 2006/25/EC of the European Parliament and of the Council (artificial optical radiation). J Eur Union 114: 38-59

Deverman BE, Pravdo PL, Simpson BP, Kumar SR, Chan KY, Banerjee A, Wu W-L, Yang B, Huber N, Pasca SP \& Gradinaru V (2016) Cre-dependent selection yields AAV variants for widespread gene transfer to the adult brain. Nat. Biotechnol. 34: 204-209

Dieter A, Duque-Afonso CJ, Rankovic V, Jeschke M \& Moser T (2019) Near physiological spectral selectivity of cochlear optogenetics. Nat. Commun. 10: 1962

Dieter A, Keppeler D \& Moser T (2020) Towards the optical cochlear implant: optogenetic approaches for hearing restoration. EMBO Mol. Med. n/a: e11618

Dombrowski T, Rankovic V \& Moser T (2019) Toward the Optical Cochlear Implant. Cold Spring Harb. Perspect. Med. 9: a033225

Duarte MJ, Kanumuri VV, Landegger LD, Tarabichi O, Sinha S, Meng X, Hight AE, Kozin ED, Stankovic KM, Brown MC \& Lee DJ (2018) Ancestral Adeno-Associated Virus Vector Delivery of Opsins to Spiral Ganglion Neurons: Implications for Optogenetic Cochlear Implants. Mol. Ther. 26: 1931-1939

Evans EF (1981) The Dynamic Range Problem: Place and Time Coding at the Level of Cochlear Nerve and Nucleus. In Neuronal Mechanisms of Hearing, Syka J \& Aitkin L (eds) pp 69-85. Boston, MA: Springer US Available at: http://link.springer.com/10.1007/978-1-4684-3908-3_9 [Accessed October 9, 2018]

Furman AC, Kujawa SG \& Liberman MC (2013) Noise-induced cochlear neuropathy is selective for fibers with low spontaneous rates. J. Neurophysiol. 110: 577-586 
Goldberg JM, Brown PB (1969) Response of binaural neurons of dog superior olivary complex to dichotic tonal stimuli: some physiological mechanisms of sound localization. $J$ Neurophysiol. 32:613-636

Gradinaru V, Zhang F, Ramakrishnan C, Mattis J, Prakash R, Diester I, Goshen I, Thompson KR \& Deisseroth K (2010) Molecular and Cellular Approaches for Diversifying and Extending Optogenetics. Cell 141: 154-165

Grieger, J. C., Choi, V. W., \& Samulski, R. J. (2006) Production and characterization of adeno-associated viral vectors. Nature protocols, 1:1412-1428

Hernandez VH, Gehrt A, Reuter K, Jing Z, Jeschke M, Mendoza Schulz A, Hoch G, Bartels M, Vogt G, Garnham CW, Yawo H, Fukazawa Y, Augustine GJ, Bamberg E, Kügler S, Salditt T, de Hoz L, Strenzke N \& Moser T (2014) Optogenetic stimulation of the auditory pathway. J. Clin. Invest. 124: $1114-1129$

Hillery CM, Narins PM (1987) Frequency and time domain comparison of low-frequency auditory fiber responses in two anuran amphibians. Hear Res. 25:233-248

Hofherr A, Fakler B \& Klöcker N (2005) Selective Golgi export of Kir2.1 controls the stoichiometry of functional Kir2.x channel heteromers. J. Cell Sci. 118: 1935-1943

Huet A, Batrel C, Tang Y, Desmadryl G, Wang J, Puel J-L \& Bourien J (2016) Sound coding in the auditory nerve of gerbils. Hear. Res.

Huet AT \& Rankovic V (2021) Application of Targeting-Optimized Chronos for Stimulation of the Auditory Pathway. Methods Mol. Biol. Clifton NJ 2191: 261-285

Keppeler D, Merino RM, Morena DL de la, Bali B, Huet AT, Gehrt A, Wrobel C, Subramanian S, Dombrowski T, Wolf F, Rankovic V, Neef A \& Moser T (2018) Ultrafast optogenetic stimulation of the auditory pathway by targeting-optimized Chronos. EMBO J. 37: e99649

Keppeler D, Schwaerzle M, Harczos T, Jablonski L, Dieter A, Wolf B, Ayub S, Vogl C, Wrobel C, Hoch G, Abdellatif K, Jeschke M, Rankovic V, Paul O, Ruther P \& Moser T (2020) Multichannel optogenetic stimulation of the auditory pathway using microfabricated LED cochlear implants in rodents. Sci. Transl. Med. 12: eabb8086

King J, Shehu I, Roland JT, Svirsky MA \& Froemke RC (2016) A physiological and behavioral system for hearing restoration with cochlear implants. J. Neurophysiol. 116: 844-858

Kleinlogel S, Vogl C, Jeschke M, Neef J \& Moser T (2020) Emerging approaches for restoration of hearing and vision. Physiol. Rev. 
Kral A, Hartmann R, Mortazavi D \& Klinke R (1998) Spatial resolution of cochlear implants: the electrical field and excitation of auditory afferents. Hear. Res. 121: 11-28

Lalwani AK, Walsh BJ, Reilly PG, Muzyczka N, Mhatre AN (1996) Development of in vivo gene therapy for hearing disorders: introduction of adeno-associated virus into the cochlea of the guinea pig. Gene Ther.; 3:588-592.

Lenarz T (2018) Cochlear implant - state of the art. GMS Curr. Top. Otorhinolaryngol. Head Neck Surg. 16: Available at: https://www.ncbi.nlm.nih.gov/pmc/articles/PMC5818683/ [Accessed July 31, 2018]

Liberman MC (1978) Auditory-nerve response from cats raised in a low-noise chamber. J. Acoust. Soc. Am. 63: 442-455

Mager T, Morena DL de la, Senn V, Schlotte J, D’Errico A, Feldbauer K, Wrobel C, Jung S, Bodensiek K, Rankovic V, Browne L, Huet A, Jüttner J, Wood PG, Letzkus JJ, Moser T \& Bamberg E (2018) High frequency neural spiking and auditory signaling by ultrafast red-shifted optogenetics. Nat. Commun. 9: 1750

Miller CA, Abbas PJ, Robinson BK, Nourski KV, Zhang F \& Jeng F-C (2006) Electrical Excitation of the Acoustically Sensitive Auditory Nerve: Single-Fiber Responses to Electric Pulse Trains. J. Assoc. Res. Otolaryngol. 7: 195-210

Richter C-P, Rajguru SM, Matic AI, Moreno EL, Fishman AJ, Robinson AM, Suh E \& Walsh JT (2011) Spread of cochlear excitation during stimulation with pulsed infrared radiation: inferior colliculus measurements. J. Neural Eng. 8: 056006

Sachs MB \& Abbas PJ (1974) Rate versus level functions for auditory-nerve fibers in cats: tone-burst stimuli. J. Acoust. Soc. Am. 56: 1835-1847

Sahel J \& Roska B (2013) Restoring vision. Nature 557: 359-367

Shannon RV, Cruz RJ \& Galvin JJ (2011) Effect of Stimulation Rate on Cochlear Implant Users' Phoneme, Word and Sentence Recognition in Quiet and in Noise. Audiol. Neurotol. 16: 113-123

Stockklausner C, Ludwig J, Ruppersberg JP \& Klöcker N (2001) A sequence motif responsible for ER export and surface expression of Kir2.0 inward rectifier K(+) channels. FEBS Lett. 493: 129-133

Taberner AM \& Liberman MC (2005) Response Properties of Single Auditory Nerve Fibers in the Mouse. J. Neurophysiol. 93: 557-569

Viemeister NF \& Bacon SP (1988) Intensity discrimination, increment detection, and magnitude estimation for 1-kHz tones. J. Acoust. Soc. Am. 84: 172-178 
Weiss RS, Voss A \& Hemmert W (2016) Optogenetic stimulation of the cochlea-A review of mechanisms, measurements, and first models. Netw. Comput. Neural Syst. 27: 212-236

Wen B, Wang GI, Dean I \& Delgutte B (2012) Time course of dynamic range adaptation in the auditory nerve. J. Neurophysiol. 108: 69-82

Winter IM, Robertson D \& Yates GK (1990) Diversity of characteristic frequency rate-intensity functions in guinea pig auditory nerve fibres. Hear. Res. 45: 191-202

Wrobel C, Dieter A, Huet A, Keppeler D, Duque-Afonso CJ, Vogl C, Hoch G, Jeschke M \& Moser T (2018) Optogenetic stimulation of cochlear neurons activates the auditory pathway and restores auditory-driven behavior in deaf adult gerbils. Sci. Transl. Med. 10: eaao0540

Zeng FG (2017) Challenges in Improving Cochlear Implant Performance and Accessibility. IEEE Trans. Biomed. Eng. 64: 1662-1664

Zeng F-G \& Galvin JJI (1999) Amplitude Mapping and Phoneme Recognition in Cochlear Implant Listeners. Ear Hear. 20: 60-74

Zierhofer CM, Hochmair-Desoyer IJ \& Hochmair ES (1995) Electronic design of a cochlear implant for multichannel high-rate pulsatile stimulation strategies. IEEE Trans. Rehabil. Eng. 3: 112-116

Zolotukhin, S., Byrne, B. J., Mason, E., Zolotukhin, I., Potter, M., Chesnut, K., Summerford, C., Samulski, R. J., \& Muzyczka, N. (1999). Recombinant adeno-associated virus purification using novel methods improves infectious titer and yield. Gene therapy, 6:973-985

Acknowledgments: We thank Daniela Gerke, Sandra Gerke and Christiane Senger-Freitag for expert technical assistance.

Funding: This work was funded by the European Research Council through the Advanced Grant 'OptoHear" to T.M. under the European Union's Horizon 2020 Research and Innovation program (grant agreement No. 670759) and was further supported by the German Research Foundation through the Cluster of Excellence (EXC2067) Multiscale Bioimaging (T.Ma.) as well as the Leibniz Program to T.M., and a scholarship of the Göttingen Promotionskolleg für Medizinstudierende, funded by the Jacob-Henle-Programm or ElseKröner-Fresenius-Stiftung (Promotionskolleg für Epigenomik und Genomdynamik, 2017_Promotionskolleg.04) to A.M.

Author contributions: B.B. designed the study, performed o/aABR and single SGNs recordings, immunohistochemistry and FP expression analysis. D.L.M. performed recordings from single SGNs and oABRs. V.R. designed the study, performed cloning, production and 
injections of the viruses. A.H. analyzed the data and designed the study. T.M designed the study and supervised the work. All authors contributed to the study design, analyzing of the data, contributed to the writing of the manuscript.

Competing interests: The authors declare no competing interests. 


\section{Conclusion}

In this chapter, we found that red-shifted ultrafast Chrimson variants are suitable for future oCI: both f-Chrimson and vf-Chrimson drive the auditory nerve at high stimulation rates with satisfying fidelity. This will allow for precise encoding temporal features of sound. When fChrimson and vf-Chrimson are compared, the latter did not appear as a much faster option at the single unit level while oABR measurements, representing the neural population response, suggests this to be the case. Moreover, between the two versions of vf-Chrimson, enhanced membrane targeting of the opsin improved sensitivity to light, yet in terms of response magnitude, it did not boost oABR wave I amplitude as much as it did for Chronos. Finally, fChrimson showed a larger operating range compared to electrical stimulation (Miller et al., 2006).

Fast-Chrimson has short light on-/off-cycle meanwhile still quite light sensitive which would enable improved encoding of frequency and amplitude of sound, respectively. It has also a large expression level and is localized in cell membrane of the SGNs. Furthermore, in mouse, expression of f-Chrimson, and hence its functionality, endures at least up to nine months (Mager et al., 2018). At this point, f-Chrimson can emerge as the opsin of choice for future clinical oCI. As the last checkpoint before clinical applications, in the next chapter, I tried to understand the possible effects of viral f-Chrimson delivery into the mouse cochlea over a time course. 


\section{Chapter 3: \\ Nonclinical biosafety assessment of red-shifted cochlear optogenetics}

\section{Introduction}

Optogenetics in the field of neuroscience has paved the way for precise spatial activation/inhibition of neuronal populations almost two decades ago (Boyden et al, 2003). In terms of cochlear optogenetics, it enabled optical control of auditory nerve in various rodents (Hernandez et al, 2014; Wrobel et al, 2018). In gerbils, cochlear optogenetics showed also narrower activation of the auditory nerve promising for better place-code of sound (Dieter et al, 2019; 2020). Moreover, behaviorally it enabled percepts in gerbils and rats (Wrobel et al, 2018; Keppeler et al, 2020; Jablonski et al, 2020).

Cochlear optogenetics requires a genetic modification in the AN using foreign microbial opsins. For future applications, this genetic modification must be efficient and stable to provide users reliable hearing over years without any harmful effects. As in any drug development or gene therapy trials, we share the concerns of such manipulation in terms of cellular specificity and safety in terms of possible unwanted degeneration and immunological reaction at on- and off-target tissues.

A previous study investigated the expression of microbial opsin ChR-2 using AAV-based technology in the retina and their effects in the neural tissue (Sugano et al, 2010). Their aim was to restore vision in blind rats. In this long-term work over year, it was possible to elicit reliable physiological responses. After a year of expression, authors reported little or no pathological indications or immunological response caused by neither ChR-2 expression nor AAV2. However, local delivery was systemically disseminated such that intestine, lung and heart tissue were shown to be positive for ChR-2 transcripts.

AAV-based gene delivery promises safe way of therapy (Kleinlogel et al, 2020). It provides non-pathogenic gene delivery to target organs. Recombinant AAV vectors are mostly nonintegrating into the host genome and it can still achieve efficient long-term expression of the gene of interest. AAV-based gene delivery technologies are already in medical use for gene therapy. In the last decade, there are three such medications approved by the European Medicines Agency and the US Food and Drug Administration. These are Glybera, LUXTURNA and ZOLGENSMA used for the treatment of genetic diseases lipoprotein lipase deficiency, inherited retinal disease, and spinal muscular atrophy (Nidetz et al, 2020). Here, 
LUXTURNA provides healthy copies of RPE65 gene to the retinal cells as a therapy to blinding retinal dystrophy (Rodrigues et al, 2018).

Here, we wanted to assess efficiency, stability and safety of AAV-based optogenetic transduction of mouse auditory nerve considering the potential translational studies developing oCI systems. Viral construct of interest was chosen as AAV2/6 capsid carrying coding sequence fast-Chrimson under the neuron-specific human synapsin promoter. We opt for this since this opsin is (1) physiologically suitable to response properties of auditory nerve and (2) after 9-months of expression, the AN can still be reliably driven. It shows (3) enhanced cell membrane localization without additional trafficking signals and (4) it has redshifted absorption/activation spectrum which makes it more favorable for lesser phototoxicity.

\section{Materials and Methods}

\section{Animals}

Wildtype C57BL/6J mice of either sex were used in this study. Animals were then kept in a $12 \mathrm{~h}$ light/dark cycle, with access to food and water ad libitum. All experiments were done in compliance with the German national animal care guidelines and were approved by the board for animal welfare of the University Medical Center Göttingen and the animal welfare office of the state of Lower Saxony.

\section{Viral construct and delivery}

Viral construct and delivery procedures were done exactly as in Mager et al., 2018. Viral construct was prepared by UNC Virus Core (vector genome: 5 x 109). Briefly, postnatal day 6 mouse pups were unilaterally injected through the round window membrane into the scala tympani. All injections were done by Dr. Vladan Rankovic and Mrs. Christiane SengerFreitag.

\section{Organ collection}

Under deep isoflurane anesthesia, the mice were decapitated to dissect whole brain, cochleae, heart, kidney, liver, lung and spleen. The brains were directly immersed into $4 \%$ paraformaldehyde and left for fixation for 24 hours at $+4{ }^{\circ} \mathrm{C}$. After fixation, for cryoprotection, the brains were incubated in 10\%, 20\% and 30\% sucrose solutions for 24 hours at $+4{ }^{\circ} \mathrm{C}$, respectively. Once the brains were fully sunken in the final $30 \%$ sucrose solution, they were embedded in cube molds using optimal cutting temperature medium. The brains were then flash-frozen in liquid nitrogen and stored in $-80{ }^{\circ} \mathrm{C}$ until further sectioning. 
After dissecting the whole brain out of skull, inner and middle ear structures, i.e. cochlea, vestibular system and the ossicular chain, were gently removed from the respective temporal bones using fine-tip forceps. The protective bony bulla was also removed to visualize the snail shape and the round window of the cochlea. Lastly, five inner organs were sampled and frozen to $-20{ }^{\circ} \mathrm{C}$ until further PCR analysis.

\section{Histology}

Cochleae: The procedure described in Mager et al, 2018, was followed. 4\% FA fixation, 0.12M EDTA decalcification, 25\% glucose cryoprotection steps were followed, respectively. $0.16 \mu \mathrm{m}$ thick cross-sections across modiolus were collected on a cryostat. Sections were stained using guinea pig anti-parvalbumin (1:300; SySy) and chicken anti-GFP mouse (1:500; Abcam) primary antibodies, goat anti-guinea pig 568 and goat anti-chicken 488 (1:200, ThermoFisher) secondary antibodies, imaging was done on inverted SP5 confocal microscope (Leica).

Brain: Brain histology was done by Dr. Eva Gruber-Dujardin at the Pathology Department of German Primate Center. Since initial histology was done on cryosections did not work, we switched to paraffin-embedded histology. Brains were fixed in $4 \%$ formaldehyde solution and divided into three coronal blocks: Block I, II and III contained frontal, middle and hind brain regions, respectively. 2-3 $\mu \mathrm{m}$ thick sections were collected on positively charged slides where each slide contained one paraffin section from each block. The consecutive sections were stained for chicken anti-GFP antibody (1:500, Abcam), mouse anti-human CD3 (1:50, Agilent), mouse anti-IBA1 (1:100, Genetex) primary antibodies respectively. For all markers, Discovery universal secondary antibody (Roche) was used along with DAB Map Kit (Roche) for chromogenic labeling. All sections were stained with hematoxylin as background label. All staining procedure was done on Ventana Discovery Staining Platform. Image acquisition of the slides was done DAB-stained slides were scanned with the Aperio CS2 scanner at a magnification of $20 \mathrm{X}$ (Leica, Germany).

\section{SGN transduction and density}

Using ImageJ, SGN cell bodies were first counted on magenta parvalbumin channel and they were counted on green YFP one at maximum intensity z-project. Average intensity values from spiral limbus were subtracted from the whole image to reduce background autofluorescence. All statistical testing and display of data was done on GraphPad Prism. Transduction level then was determined as: 
(Number of double positive SGN somata

$\div$ Number of parvalbumin positiveSGN somata) $\times 100$

SGN densities were calculated as:

Number of parvalbumin positive somata $\div$ Area of Rosenthal's canal in $10^{4} \mu m^{2}$

Semi-quantitative analysis of $f$-Chrimson-YFP expression in brain

The digitized images were analyzed with Aperio ImageScope (Version 12.4.3.5008, Leica Biosystems). Block I and II were annotated into 4 segments as left-right and corticalsubcortical, block III were done as left-right and cerebellar-brainstem. Upon visual inspection, scores were given in a range of 0-4 where

0: $0 \%$ = YFP positivity;

1: $0 \%<$ YFP positivity $\leq 10 \%$;

2: $10 \%<$ YFP positivity $\leq 40 \%$;

3: $40 \%<$ YFP positivity $\leq 85 \%$;

4: $85 \%<$ YFP positivity $\leq 100 \%$

\section{T-cell counts}

Using "Counter Tool" function of Aperio ImageScope, T-cells were counted in each annotated area, for example left hemi-cerebellum, separately. Then, number of cells was divided by the area of annotated area in $\mathrm{mm}^{2}$.

\section{Microglia activation analysis}

Using built-in "Positive Pixel Count v9" analysis algorithm, number of positive and negative pixels, and area of annotations were extracted. The algorithm makes use Munsell color scheme (Munsell, 1905) where color is defined in three parameters: hue being basic color; chroma being color intensity and value notifying lightness/brightness. For brown, color of chromogen used in this study, hue is given as 0.1 by definition in this algorithm. Chroma, or given as hue width in the program, can be set to $0.33-0.5$ which then includes pixels having colors of red and yellow. I have set this to 0.33 . Finally, all brown pixels are categorized into three according to their brightness, i.e. weakly positive brown pixels: $220-175$; positive brown 
pixels: 175-100; strongly positive brown pixels: 100-0. All pixels which are not brown but blue are classified as negative pixels (mostly nuclei). After obtaining the number of positive and negative pixels, we defined three measures to be able to compare experimental groups and determine microglial activation.

Positivity defined as:

Number of all positive pixels / Number of all negative pixels

Microglial activation defined as:

$$
\begin{gathered}
\text { Number of strongly positive pixels /(Number of positive pixels } \\
+ \text { Number of weakly positive pixels) }
\end{gathered}
$$

Microglial activation per $\mu \mathrm{m}^{2}$ defined as:

$$
\text { Number of strongly positive pixels /Annotated area in } \mu m^{\wedge} 2
$$

\section{PCR detection}

DNA isolation was done using genomic DNA isolation kit from tissue and cells (Nexttec) according to user's manual. The designed primers targeted downstream of f-Chrimson coding sequence and upstream of YFP coding sequence. Forward primer: 5'TTGGGGCATGTACCCAATCC-3'; Reverse primer: 5'-TCTCGTTGGGGTCTTTGCTC-3'. All DNA isolation and PCR protocols were run by Mrs. Christiane Senger-Freitag. 


\section{Results}

\section{Expression of f-Chrimson in SGNs lasts for two years}

Mager and colleagues (2018) have shown that postnatally transduced mouse SGNs can express the f-Chrimson construct up to at least 9 months of age allowing reliable orange-light activation of auditory nerve. In the same study, the younger age group covered a rather large time window ( 1-4 months old). Here, I tested the expression level and longevity of $\mathrm{f}$ Chrimson in the SGNs at several time points over 24 months. C57B1/6J mice pups (p6) were injected unilaterally through round window membrane using the same batch of viral preparation, i.e. AAV2/6 hSyn-f-Chrimson-EYFP, as described in Mager et al., 2018. The mice were euthanized for organ sampling at the ages of 1, 3, 6, 12, and 24 months. Fluorescent immunohistochemistry with parvalbumin (which, unlike calretinin (Shrestha et al., 2018), detects most if not all type I SGNs) and GFP antibodies on the cochlear cryosections across three modiolar turns (apical, middle, basal) was used to label total and transduced SGN populations, respectively (Figure 3.1A). Transduction levels reached up to $80 \%$ in the injected cochlea for all the age groups (Figure 3.1B) and they were all similar (Tukey's multiple comparisons test; 3 vs 1 month old, $\mathrm{p}=0.1749 ; 6$ vs 1 month old, $\mathrm{p}=9570$; 12 vs 1 month old; $\mathrm{p}=9942 ; 24$ vs 1 month old, $\mathrm{p}=2449 ; 6$ vs 3 months old, $\mathrm{p}=0.9346 ; 12$ vs 3 months old, $p=0.6099 ; 24$ vs 3 months old; $p>0.9999 ; 12$ vs 6 months old, $p$ > 0.9999; 24 vs 6 months old, $p=0.9683 ; 24$ vs 12 months old, $p=0.7223$ ). Yet, expression of the $f-$ Chrimson construct was not limited to the injected cochlea only. While much lower compared to the injected side (ordinary two-way ANOVA, $\mathrm{p}<0.0001$ ), the transduction level was as high as $\sim 8 \%$ (24-month-old) in the contralateral non-injected cochlea. Finally, when distribution of transduction among modiolar turns was analyzed (Figure 3.1C), apical and middle turn SGNs were at higher levels of transduction $(60 \%-80 \%)$ compared to basal ones $(\sim 40 \%$, Kruskal-Wallis test, $\mathrm{p}=0.0005)$. Even though expression levels across the modiolar turns vary, similar grand averages (across all turns, black dots, Figure 3.1C) among different age groups indicate that a big proportion of SGNs maintain expression of the f-Chrimson construct over the average mouse lifetime of two years. 


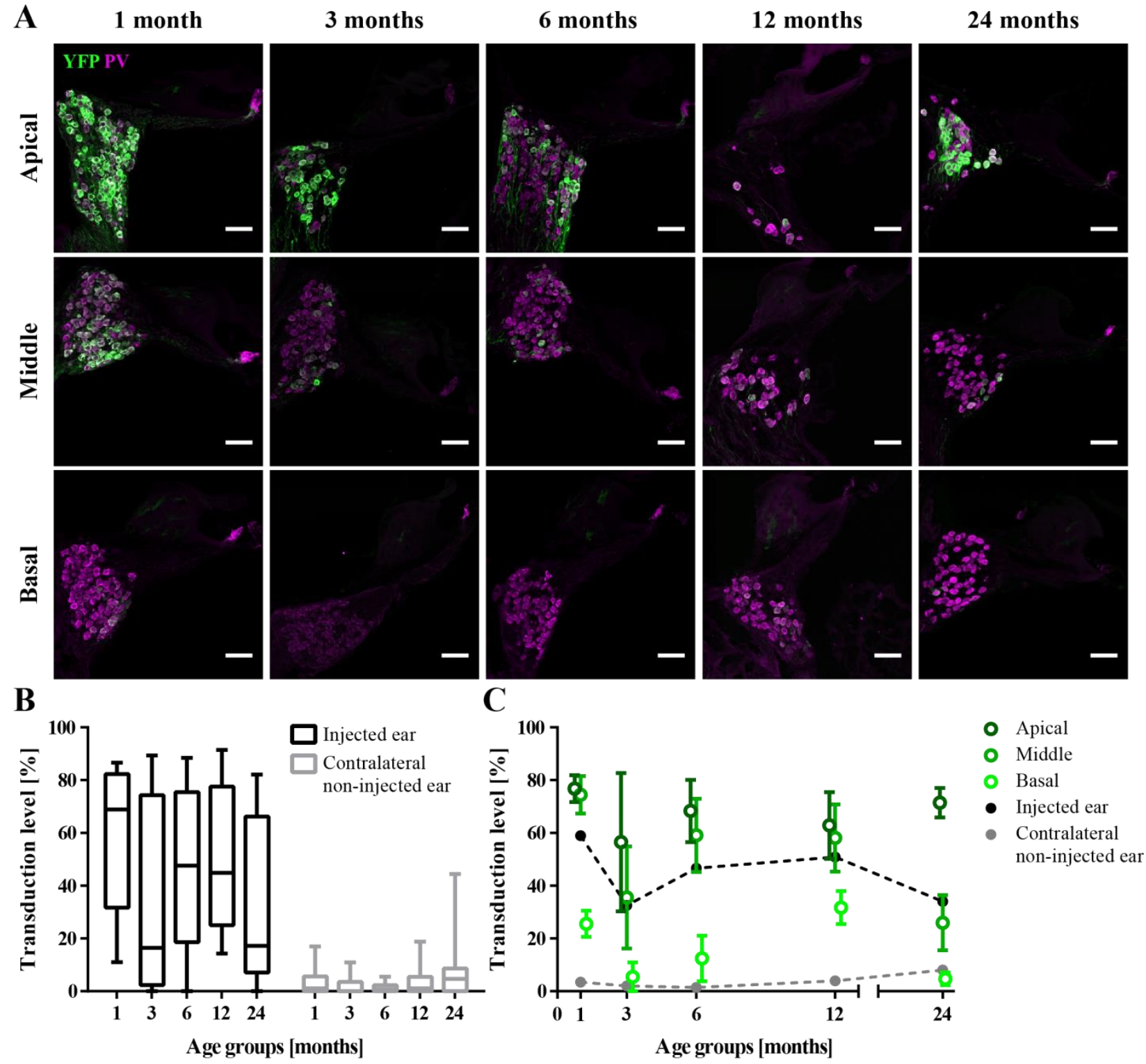

Figure 3.1: Expression of f-Chrimson in transduced mouse cochlea.

(A) Exemplary confocal images of mid-modiolar sections from cochleae injected with the AAV-f-Chrimson for the different age groups. Scale bar: $50 \mu \mathrm{m}$. (B) Transduction levels of SGNs across all cochlear turns from the injected and contralateral non-injected cochleae for the age groups. Box-whisker plots with upper and lower limits of the boxes representing 75\% and $25 \%$ of the dataset and the whiskers $90 \%$ and $10 \%$, respectively. Horizontal lines in the boxes indicate the median of the corresponding dataset. $\mathrm{N}_{1 \text {-month }}=4, \mathrm{~N}_{3 \text {-month }}=3, \mathrm{~N}_{6 \text {-month }}=3$, $\mathrm{N}_{12 \text {-month }}=5, \mathrm{~N}_{24-\text { month }}=3$ mice. (C) Transduction levels (mean \pm sem) at different modiolar turns are plotted among all the age groups. Grand averages (across all turns) of injected (black) and contralateral non-injected (grey) are also shown for comparison.

\section{Age- and manipulation-related SGN loss}

A great proportion of SGNs (50-60\%) maintained expression of f-Chrimson for 24 months. However, in some images, the lower number of neuron bodies e.g. apical, 12-months-old in Figure 3.1A, suggested the possibility of neuronal loss. C57B1/6J strain mice are known to start losing hearing by the age of 6 months (Kane et al., 2012). This age-related hearing loss, 
a.k.a. presbycusis, initially exhibit itself by the degeneration of inner and outer hair cells at the high-frequency sound coding base of cochlea. Yet, decrease in number of SGNs at the basal turns of cochlea starts at a later age of 15 months (Someya et al., 2009).

To better understand the degree of SGN loss associated with age and possibly with the optogenetic manipulation, we first compared the density of SGN bodies between the injected and contralateral non-injected cochleae (Figure 3.1A, 1C) which did not differ from each other (ordinary two-way ANOVA, $\mathrm{p}=0.4374$ ). Yet, age emerged as a factor for SGN loss where average SGN densities in the injected cochlea varied from 52.7, 40, 46.8, 32.5, 28.3 SGN bodies $/ 10^{4} \mu \mathrm{m}^{2}$ for 1, 3, 6, 12 and 24-month-old age groups, respectively (ordinary twoway ANOVA, $\mathrm{p}<0.0001)$. Contralateral non-injected cochleae had average densities given as 49.1, 45.2, 43.6, 45.2, $25.8 \mathrm{SGN}$ bodies $/ 10^{4} \mu \mathrm{m}^{2}$ for 1, 3, 6, 12 and 24-month-old age groups, respectively. Pairwise comparisons of SGN densities in the injected cochlea between 1versus 12-month-old and 1- versus 24-month-old showed a decrease (Tukey's multiple comparisons test, $\mathrm{p}_{1}$ vs $12=0.0003, \mathrm{p}_{1}$ vs $24<0.0001$ ). In the contralateral non-injected side, however, there was a difference in SGN density only between 1- and 24-month-old (Tukey's multiple comparisons test, $\left.\mathrm{p}_{1 \text { vs } 24}=0.0003\right)$. At the age of 12 months, when the SGN densities are compared across all turns, only the apical turn of the injected cochlea and middle turn of contralateral non-injected side were found to be different from each other (Dunn's multiple comparison test, $\mathrm{p}=0.0144)$. 
A
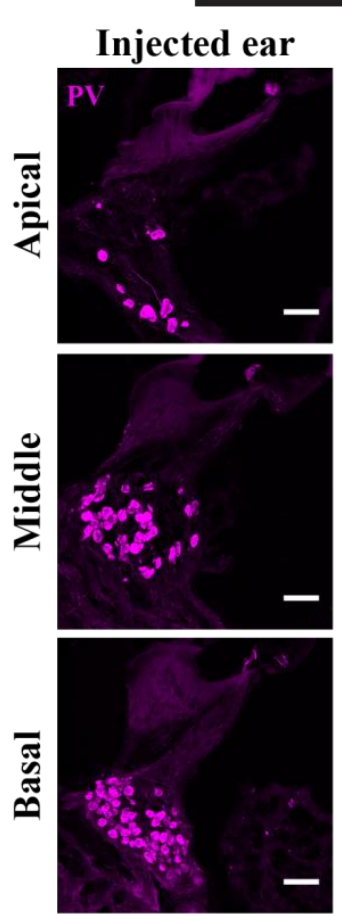

$\mathbf{C}$
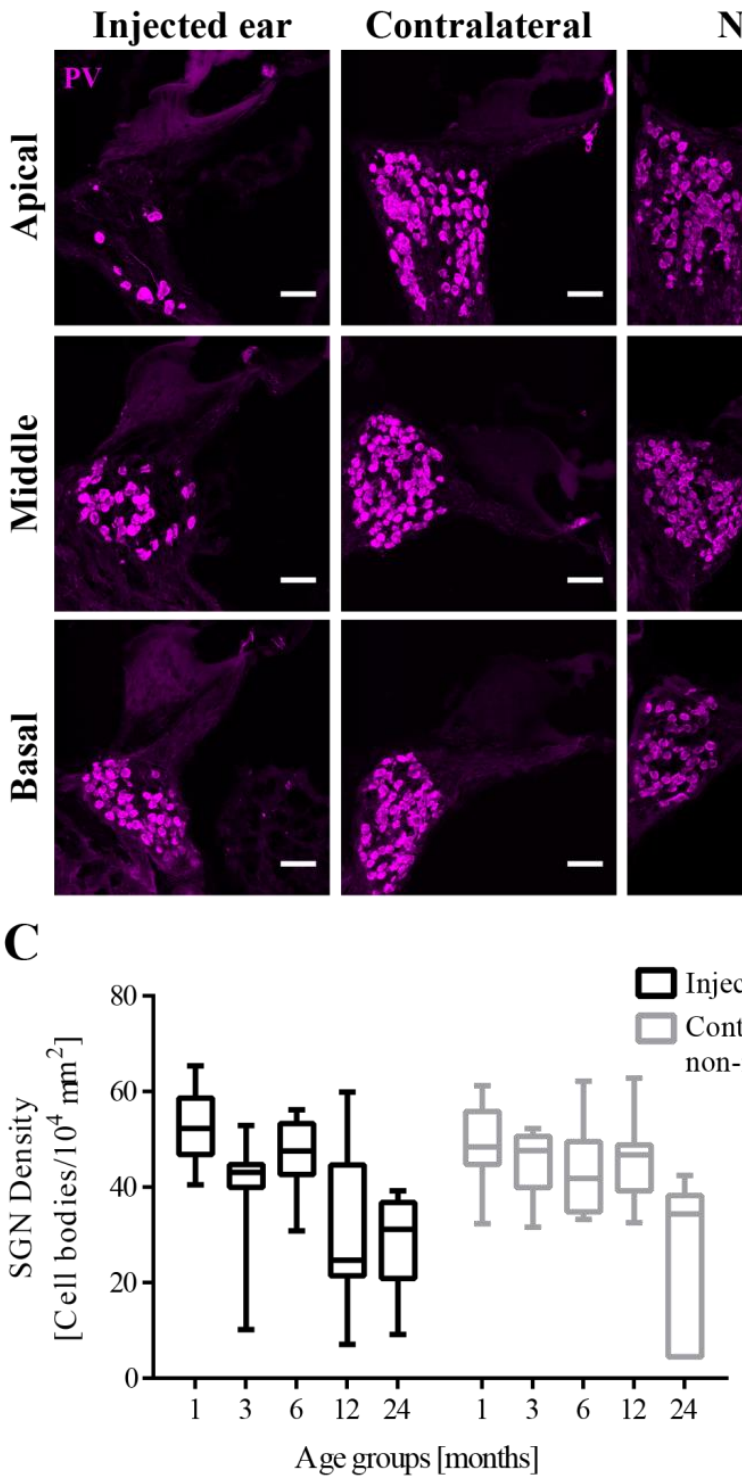

B

3 months
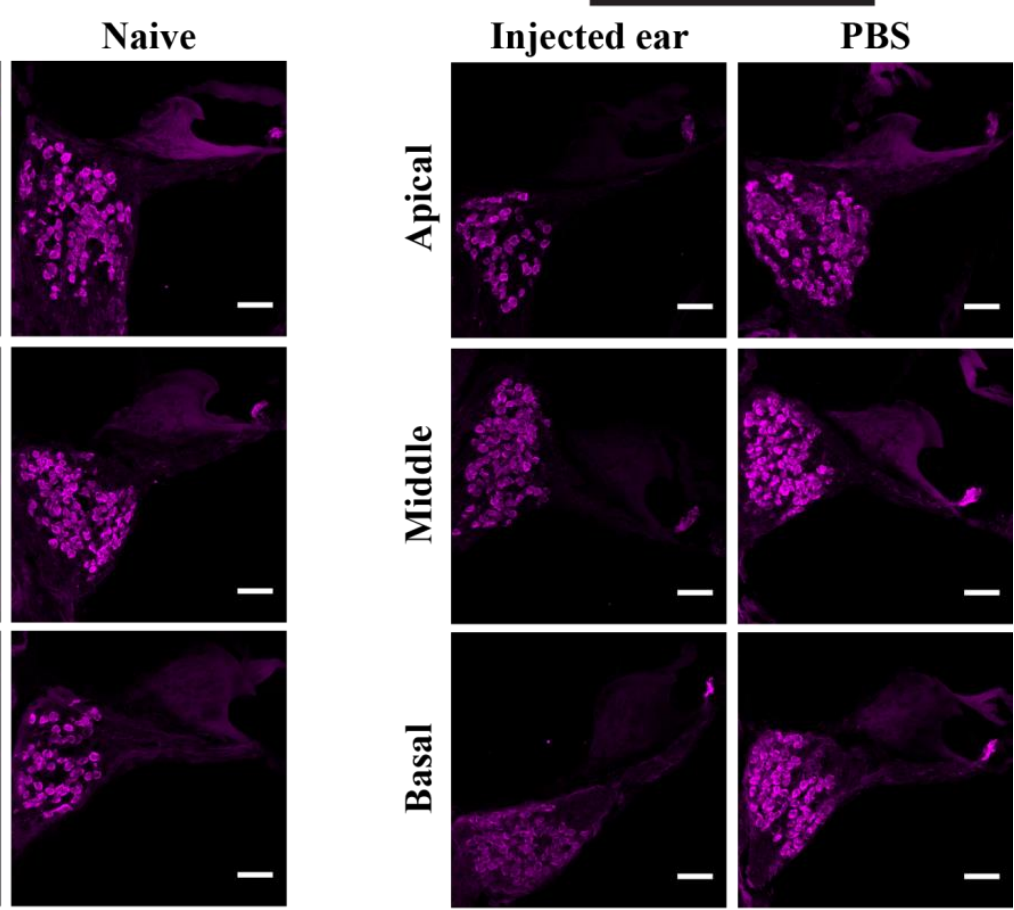

D

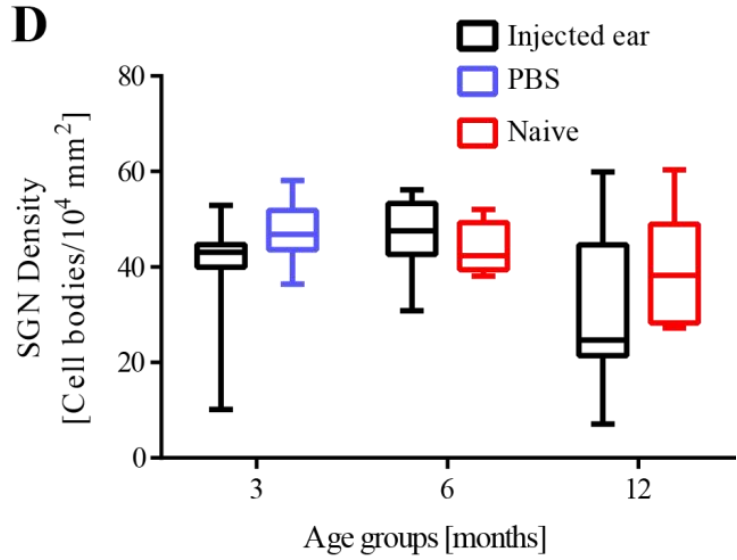

Figure3.2: SGN density profile of f-Chrimson-injected cochlea in comparison with negative controls.

(A) Confocal images of mid-modiolar cross-sections from injected, contralateral non-injected and naïve cochleae from 12-month-old mice. Scale bar: $50 \mu \mathrm{m}$. (B) Confocal images of modiolar cross-sections from injected cochleae of f-Chrimson- and PBS-injected mice at the age of 3 months. Scale bar: $50 \mu \mathrm{m}$. (C) SGN densities across all cochlear turns from the injected and contralateral non-injected cochleae across the age groups are plotted in boxwhisker graphs. Upper and lower limits of the boxes represent $75 \%$ and $25 \%$ of the dataset, respectively. Horizontal lines in the boxes indicate the median, whereas the whiskers stand for maximum and minimum of the corresponding dataset. $\mathrm{N}_{1 \text {-month }}=4, \mathrm{~N}_{3 \text {-month }}=3, \mathrm{~N}_{6 \text {-month }}=3$, $\mathrm{N}_{12 \text {-month }}=5, \mathrm{~N}_{24}$-month $=3$ mice. (D) SGN densities per any modiolar turn from the injected cochleae of AAV-f-Chrimson-, PBS-injected and naïve animals for the age groups of 3-, 6and 12-month-old mice. Border designation of the plots is as in $\mathrm{C} \mathrm{N}_{3 \text {-month }}=3, \mathrm{~N}_{6 \text {-month }}=3$, $\mathrm{N}_{12 \text {-month }}=5$ mice for AAV-f-Chrimson; $\mathrm{N}_{3 \text {-month }}=2$ mice for PBS; $\mathrm{N}_{6 \text {-month }}=2$ mice $\mathrm{N}_{12 \text {-month }}$ $=2$ mice for naïve group. 
Secondly, to elaborate more thoroughly, we compared the SGN densities of the AAV-fChrimson-injected cochleae either to PBS-injected at 3 months or to naïve cochleae at 6 and 12 months (Figure 3.2A, B, D). At the age of 3 months, average SGN density of the injected cochleae of AAV-f-Chrimson-injected mice is $40 \mathrm{SGN}$ bodies $/ 10^{4} \mu \mathrm{m}^{2}$ similar to that of PBSinjected control being 47.3 SGN bodies $/ 10^{4} \mu \mathrm{m}^{2}$ (Mann-Whitney test, $\mathrm{p}=0.0879$ ). Average SGN densities of 6- and 12-month-old naïve control groups were 43.8 and 39.5 SGN bodies $/ 10^{4} \mu \mathrm{m}^{2}$, respectively. This corroborates the average SGN densities of f-Chrimsoninjected groups, i.e. 46.8 and $32.5 \mathrm{SGN}$ bodies $/ 10^{4} \mu \mathrm{m}^{2}$ for 6 - and 12-month-old mice (Tukey's multiple comparison test; $\mathrm{p} 6$ naive vs 6 f-Chrimson $=0.9705 ; \mathrm{p} 12$ naive vs $12 \mathrm{f}$-Chrimson $=$ 0.6638). In conclusion, although aging is the main determinant of SGN loss, continuous (over)expression of f-Chrimson might pose a risk to accelerate this process.

\section{Spread of f-Chrimson expression to the brain following postnatal AAV-injection}

The above results document a lifelong expression of f-Chrimson in the SGNs in mice following a single postnatal AAV-injection into the ear. Nonetheless, significant, though lower f-Chrimson expression was also found in SGNs of the contralateral non-injected ear. A recent study injecting dye through the round window membrane into the cochlea in neonatal and adult mice, showed a spread of the dye to brain via the cochlear aqueduct (Talaei et al., 2019). Therefore, we probed for a spread of the AAV-f-Chrimson to the brain. To this end, paraffin-embedded coronal sections of the brains were collected from three blocks on the rostro-caudal axis (Figure 3.3A). Block I included mostly forebrain, block II covered the areas such as hippocampus, visual and auditory cortices, and thalamus. Cerebellum, inferior colliculi and brainstem were then collected on the block III. F-Chrimson-EYFP expression in the brain was detected using the same primary antibody as for the above fluorescence microscopy (Figure 3.1A) but employing chromogenic DAB (brown, Figure 3.3A, B) as the indicator instead.

After visual inspection of the sections, expression of f-Chrimson-YFP throughout brain was observed in postnatally AAV-f-Chrimson-injected mice (Table 3.1, Figure 3.3A). FChrimson-EYFP-expressing neurons and neural projections were present both in the neighboring regions as close as cochlear nucleus but also as far as retrosplenial cortex on the ipsilateral side (Figure 3.3B). A similar pattern of spread was also mirrored in the contralateral hemisphere, though it was less in terms of coverage and intensity (Figure 3.3A). There was no expression in naïve control animals (Figure 3.3A). 
Table3.1: Brain regions where f-Chrimson-YFP expression was most frequentlyobserved

\begin{tabular}{|c|c|}
\hline & Brain regions/structures \\
\hline Block I & $\begin{array}{l}\text { (Anterior) cingulate, (pre)limbic, orbital, primary motor and } \\
\text { somatosensory, olfactory-related such as taenia tecta and olfactory } \\
\text { tubercle }\end{array}$ \\
\hline Block II & $\begin{array}{l}\text { Retrosplenial (layer } 5 \text { and/or 6), hippocampus especially dentate } \\
\text { gyrus, visual and auditory areas, piriform, cortical-amygdalar areas, } \\
\text { thalamus, hypothalamus }\end{array}$ \\
\hline Block III & $\begin{array}{l}\text { Cerebellum, inferior colliculus, cochlear nucleus, trapezoid body, } \\
\text { olivary complex, ascending fibers to vestibular nucleus }\end{array}$ \\
\hline
\end{tabular}

We, later, did semi-quantitative scoring of the spread across the age groups (Figure 3.3C, D). Here, all groups showed minimal (0-10\%) to mild (10-40\%) level of expression along rostrocaudal axis (Figure 3.3C; ordinary two-way ANOVA, $\mathrm{p}=0.5616 ; \mathrm{N}=3$ mice per age group). Comparison between ipsi- and contralateral hemispheres also did not exhibit a prominent difference in terms of spread although ipsilateral hemisphere tended to score higher (Figure 3.3D; ordinary two-way ANOVA, $\mathrm{p}=0.0958 ; \mathrm{N}=3$ mice per age group). 
A

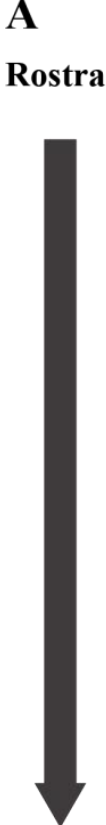

Caudal
12 months - Transduced

IPSI

흠

YFP-DAB

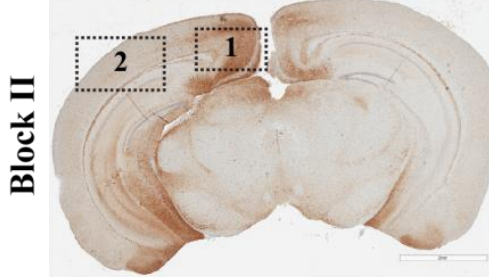

三

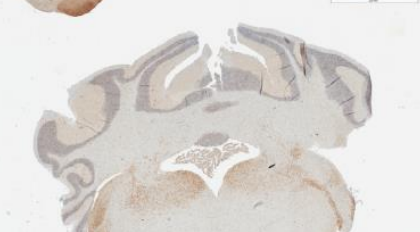

3
12 months - Naive

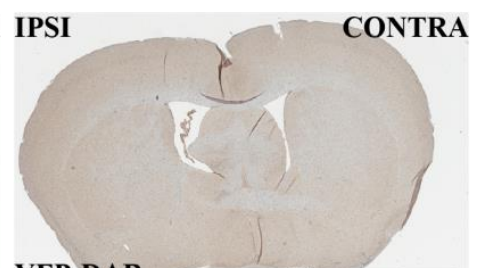

YFP-DAB

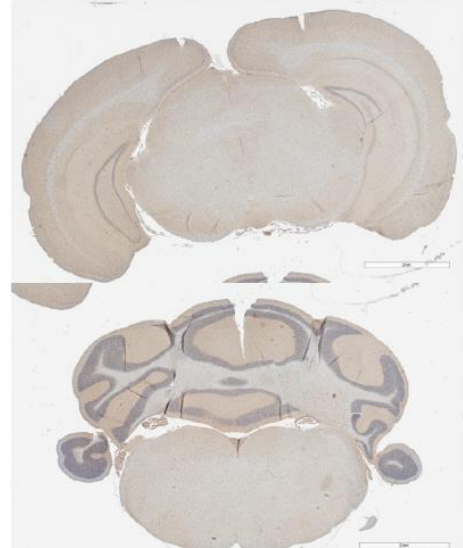

B
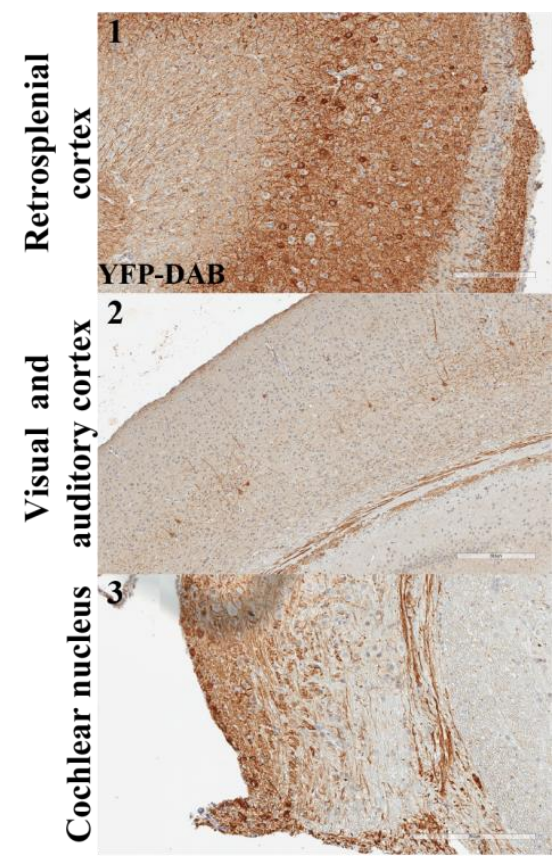

C

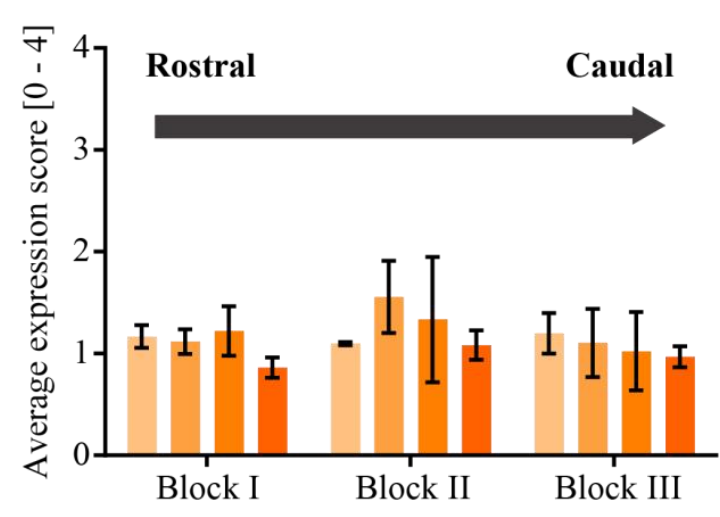

D

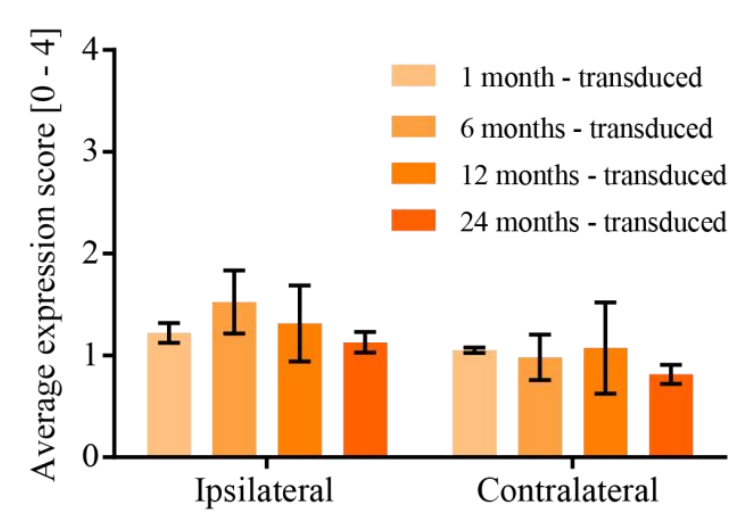

Figure3.3: Off-target expression of viral f-Chrimson construct.

(A) Exemplary light microscopy images of coronal sections of transduced and naïve mice at the age of 12 months are shown. Chromogenic DAB (brown) labels the regions where fChrimson-YFP expression is present on the three brain blocks along rostro-caudal axis. Scale bars: $2 \mathrm{~mm}$. (B) Zoom-in images of the numbered regions from (A). Scale bars: $200 \mu \mathrm{m}$. (C, D) Semi-quantitative scoring of expression of f-Chrimson along rostro-ventral (C) or mediolateral (D) axes.

\section{Inflammatory traces in brain are age-related rather than due to AAV spread}

Despite being minimal, expression of the f-Chrimson construct in brain might impose a risk of neuroinflammation considering the microbial nature of the opsin. To address this issue, we labeled consecutive coronal sections for CD-3 and IBA-1 markers. The former marks mature $\mathrm{T}$-cells and the latter is used to target at microglia. 


\section{T-lymphocyte infiltration to the neural tissue}

Brain is almost devoid of CD3-positive T-cells $\left(<1\right.$ per $\left.\mathrm{mm}^{2}\right)$ in a healthy mouse younger than 12 months old (Ferretti et al. 2016). This number can climb up to $2-3$ cells per $\mathrm{mm}^{2}$ when animal is 24 months old. In case of neurodegenerative diseases, like Alzheimer's for instance, infiltration of T-cells rises $3-4$ folds compared to age-matched mouse group. Therefore, it is a suitable indicator to pinpoint age- and f-Chrimson-YFP-related neuroinflammation.

We observed CD3-positive T-cells very rarely in the young age groups ( $<12$ months) whereas in 24-month-old group, they were abundantly found (Figure 3.4A). These cells appear as round cells with a distinct peripheral circle distributed within the neural tissue. When the brain regions having high level of f-Chrimson expression (Figure 3.3B) were checked for T-cell infiltration, these areas did not exhibit increased number of T-cells (Figure 3.4B, C). Further quantification showed that 1-, 6- and 12-month-old age animals of the AAV-injected group had $\leq 1$ cell per $\mathrm{mm}^{2}$ in which the T-cell density was doubled for 24 month-old mice along the rostro-caudal axis (ordinary two-way ANOVA, $\mathrm{p}=0.0013 ; \mathrm{N}=3$ mice per group). On the other hand, age-matched naïve controls of 6- and 12-month-old mice had a similar density of CD3-positive cells (ordinary two-way ANOVA, $\mathrm{p}=0.5015 ; \mathrm{N}=2$ mice per group) implying that increase in T-cell infiltration stems from age-related issues. 3month PBS-injected group also did not differ, for example, when compared with 1-month-old mice (ordinary two-way ANOVA, $p=0.2116$ ). T-cell densities were similar between ipsiand contralateral hemispheres (Figure 3.4D). 
A

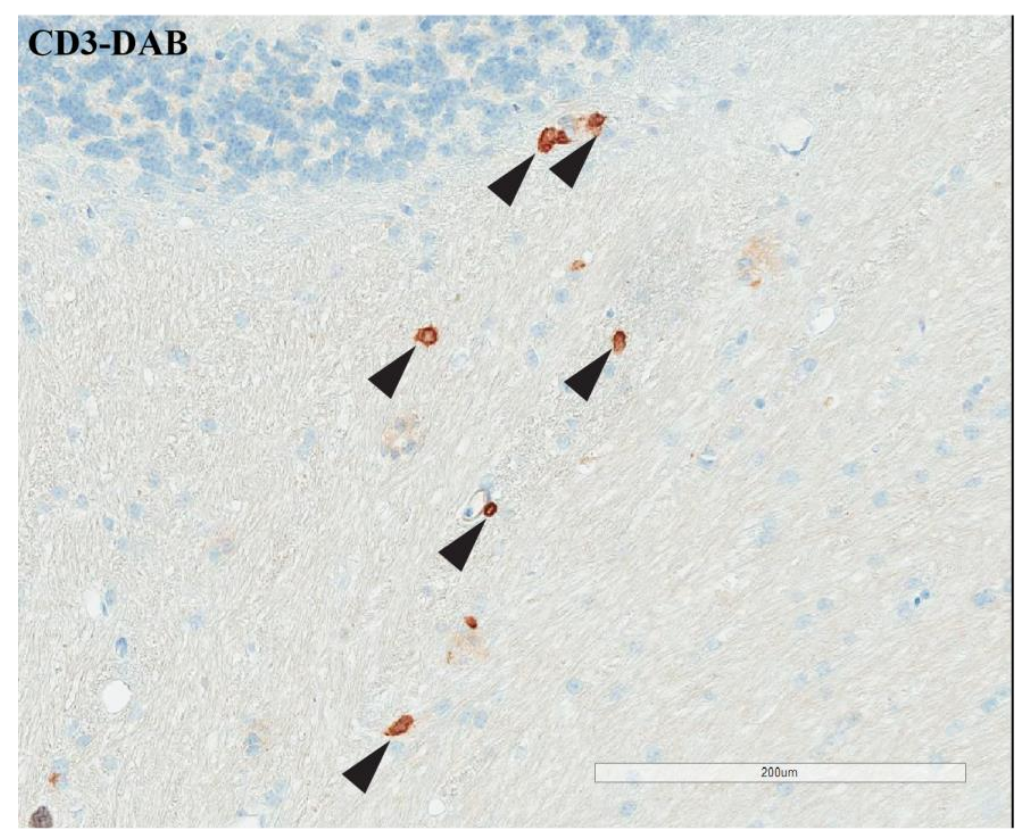

B

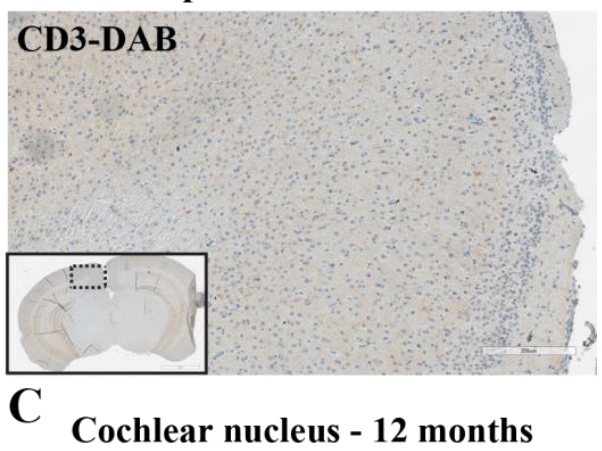

\section{CD3-DAB}

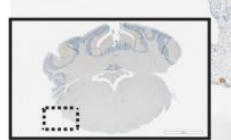

D

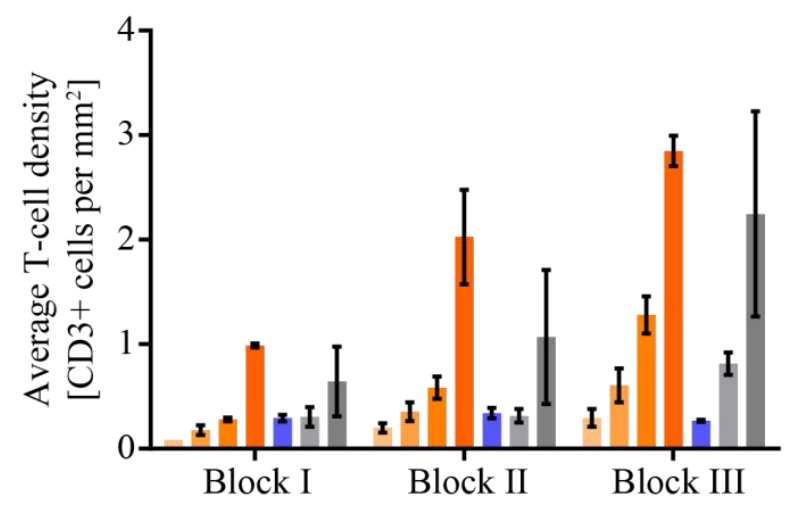

$\mathbf{E}$

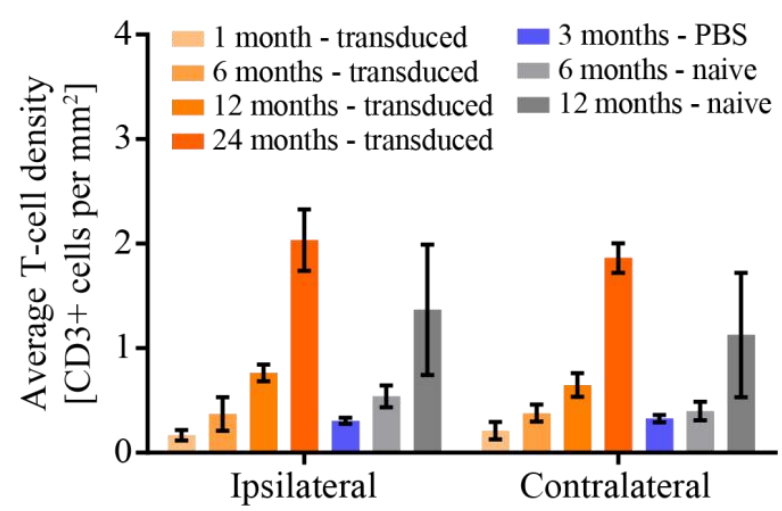

Figure3.4: T-cell infiltration in the brain

(A) Exemplary CD3-positive T-lymphocytes (arrowheads) are shown in the white matter of cerebellum from a 24-month-old mouse. Scale bar: $200 \mu \mathrm{m}$. (B, C) CD3 histology on retrosplenial cortex (B) and cochlear nucleus (C) corresponding to regions from Figure 3.3B with high-score for f-Chrimson expression. Insets show the relative location of the zoom-in image to the whole coronal section. Scale bars: $200 \mu \mathrm{m}$. (D, E) Average T-cell densities are plotted either along rostro-ventral (D) or medio-lateral (E) axes.

\section{Microglia activation as an immune response to f-Chrimson-YFP expression}

In a healthy brain, microglial cells are in a finely ramified state (arachnoid morphology). In case of neurodegenerative diseases like Alzheimer's or when there is pathogen in the neural tissue, microglial activation takes place where the cells withdraw their thin processes towards soma and become more amoeboid (Felsky et al., 2019). This morphological change reflects itself as an increase in microglial density which could be easily distinguished visually on histological samples if the inflammatory reaction is substantial. Yet, if the changes are subtle 
to eye, such mild activation would then be neglected. Here, I investigated potential microglial activation in response to $\mathrm{f}$-Chrimson-YFP expression and to aging in brain via comparing microglia density profiles.

Microglial cells were detected using IBA-1 antibodies on the inflamed positive control, and neural tissues from AAV-injected and naïve animals (Figure 3.5A, B, C). Brain samples from ring-tailed lemur (Lemur catta) under helminthic invasion were used in this study as a positive control for microglial activation and density profiling. As it is clearly visible, the density of brown IBA-labeling was drastically increased in the positive control (Figure 3.5A). In AAV-transduced and naïve animals, however, the microglia kept their arachnoid morphology (Figure 3.5B, C).

As a first step, to determine microglial distribution, positivity indices of the experimental groups were compared (Figure 3.5D, G). The positivity index represents the total number of all positive (brown, orange, yellow) pixels divided by total number of both positive and negative (grey/blue) pixels, was used as a measure of microglial coverage relative to all cell populations. Along the rostro-caudal axis (Figure 3.5D), except for block III, positivity indices were similar with each other (ordinary two-way ANOVA, $p=0.5797$ ). Ipsi- and contralateral hemispheres were also comparable in terms of positivity index (Figure 3.5G; ordinary two-way ANOVA, $\mathrm{p}=0.1770$ ). 


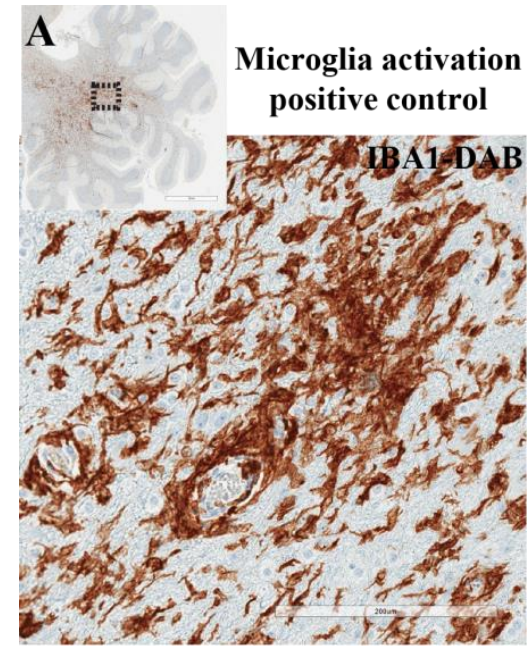

D

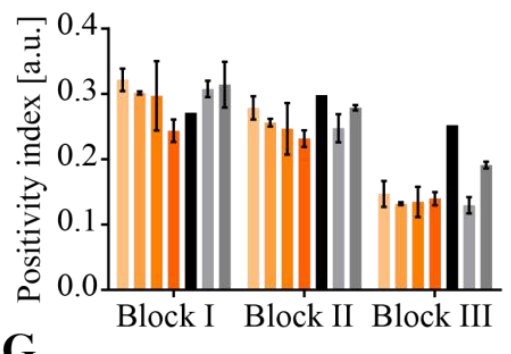

G

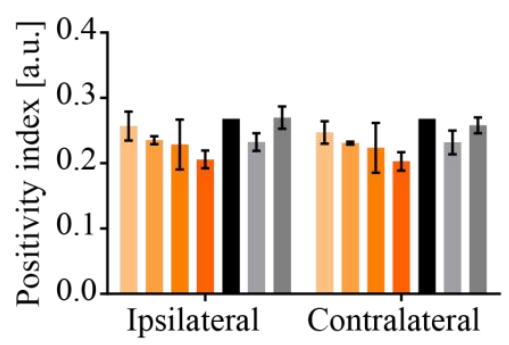

B

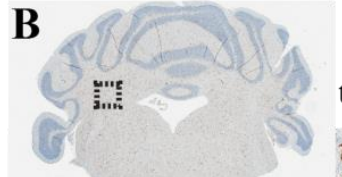

12 months transduced

IBA1-DAB

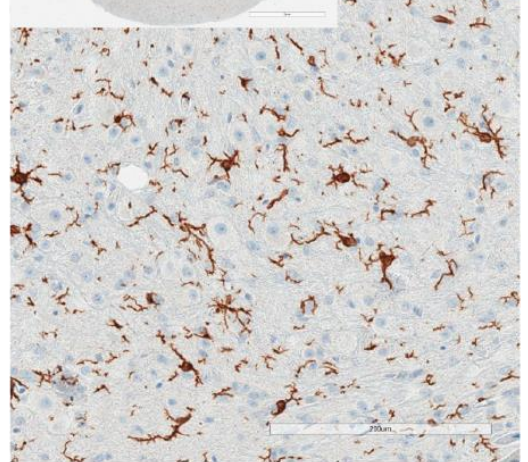

E

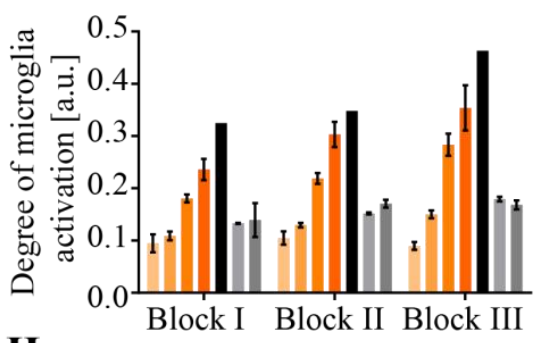

H

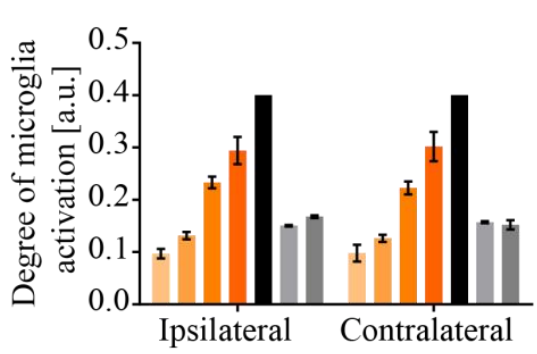

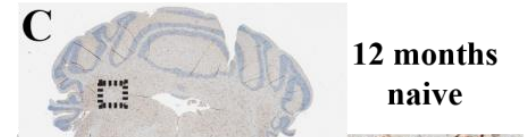

IBA1-DAAB

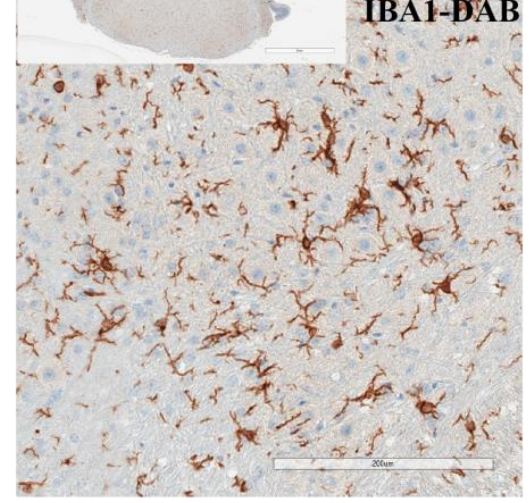

F

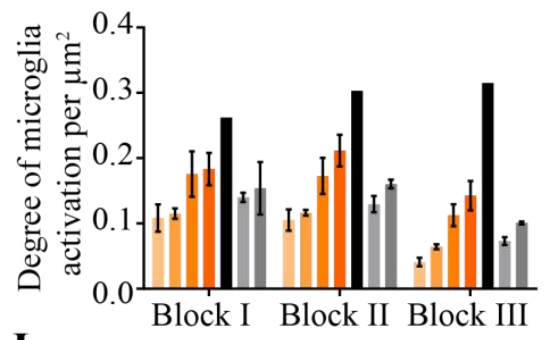

I

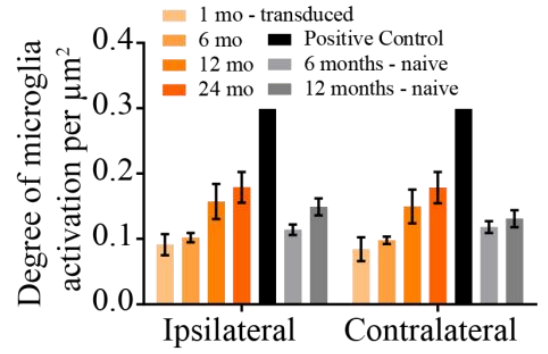

Figure3.5: Microglia activation profile

(A, B, C) Exemplary light microscopy images of IBA-1 labeled cerebellar tissue from helminth-infected (A), transduced (B), and naïve (C) animals. Insets show the overview images. Scale bars: $200 \mu \mathrm{m}$, and $2 \mathrm{~mm}$ for insets. (D, E, F) Three measures, i.e. positivity index (D), degree of microglia activation (E), degree of microglia activation per $\mu \mathrm{m}^{2}(\mathrm{~F})$, were plotted for determining rostro-caudal microglia density profile. $(\mathrm{G}, \mathrm{H}, \mathrm{I})$ The same measures $(\mathrm{D}, \mathrm{E}, \mathrm{F})$ were plotted for lateral profile.

Then, I compared the degree of microglia activation defined as total number of strongly positive pixels (brown) divided by the total sum of positive (orange) and weakly positive (yellow) pixels (Figure 3.5E, H). Here, microglial activation was referred to as proportional increase in microglia density, i.e. increase in number of brown strongly positive pixels relative to just positive and weakly positive pixels. As in T-cell infiltration, an age-related increase in microglia activation was present yet this was still milder than the positive control sample (Figure 3.5E, ordinary two-way ANOVA, $\mathrm{p}=0.0674 ; \mathrm{N}=3$ mice per transduced group, $\mathrm{N}=2$ mice per naïve group, $\mathrm{N}=1$ lemur for the positive control). Age-matched naïve 
controls also exhibited similar level of microglial activation except for 12-month-old groups of block III (Tukey's multiple comparison test, $\mathrm{p}=0.0262$ ). Laterally, the activation in hemispheres was also similar (Figure 3.5H; ordinary two-way ANOVA, p = 0.8201).

Finally, I compared the coverage of microglial activation defined as the total number strongly positive brown pixels divided by area (Figure 3.5F, I). Here, age-related microglia activation was less pronounced among transduced groups (Figure 3.5F; ordinary two-way ANOVA, p = 0.9804). Only 1- and 24-month-old age groups differed from each other at the level of block II (Tukey's multiple comparison test, $\mathrm{p}=0.0360$ ). Age-matched naïve controls were at similar degree of microglia activation as the transduced counterparts (ordinary two-way ANOVA, $\mathrm{p}=$ 0.9994). Lastly, lateral activation pattern was comparable (Figure 3.5I; ordinary two-way ANOVA, $\mathrm{p}=0.6908)$.

\section{Viral f-Chrimson construct was not detected in inner organs}

In 2011, Sugano and colleagues reported a similar study where blind rats received intravitreous injections of AAV-based channelrhodopsin-2 to restore vision in advanced retinitis pigmentosa. Although the delivery was local, in some cases, they observed expression of viral ChR-2 construct in off-target organs, i.e. intestine, lung, heart. In this study, expression of the f-Chrimson construct in the brain raised the question if this spread could be beyond neighboring tissues. Therefore, viral DNA presence was screened by conventional PCR in heart, lung, liver, kidney and spleen sampled from transduced, PBS-injected and naïve mice at different ages. A 917 basepair-long DNA strip that covered coding sequences, downstream of f-Chrimson and upstream of YFP, was targeted and amplified. The viral f-Chrimson construct was not present in the inner organs (Figure 3.6, lanes 6-10). It was detected only from the injected cochleae of the transduced animals in most cases. Only in this 1-month-old animal, PCR yielded bands for both cochleae (Figure 3.6, lanes 4-5). We used template plasmid DNA (lane 1) as a positive control that showed the expected size band around $900 \mathrm{bp}$ yet there was also a smear formation. As the positive control for DNA isolation (lane 3), primers for RIMbinding protein-2 genomic DNA was used which amplified a $\sim 350 \mathrm{bp}$ band. This gene is frequently targeted in my research group for genotyping. Lastly, the negative control (lane 2) for PCR, where distilled water was used as template interestingly, also sometimes produced a faint band around $1100 \mathrm{bp}$ which is above the expected size of $917 \mathrm{bp}$. 


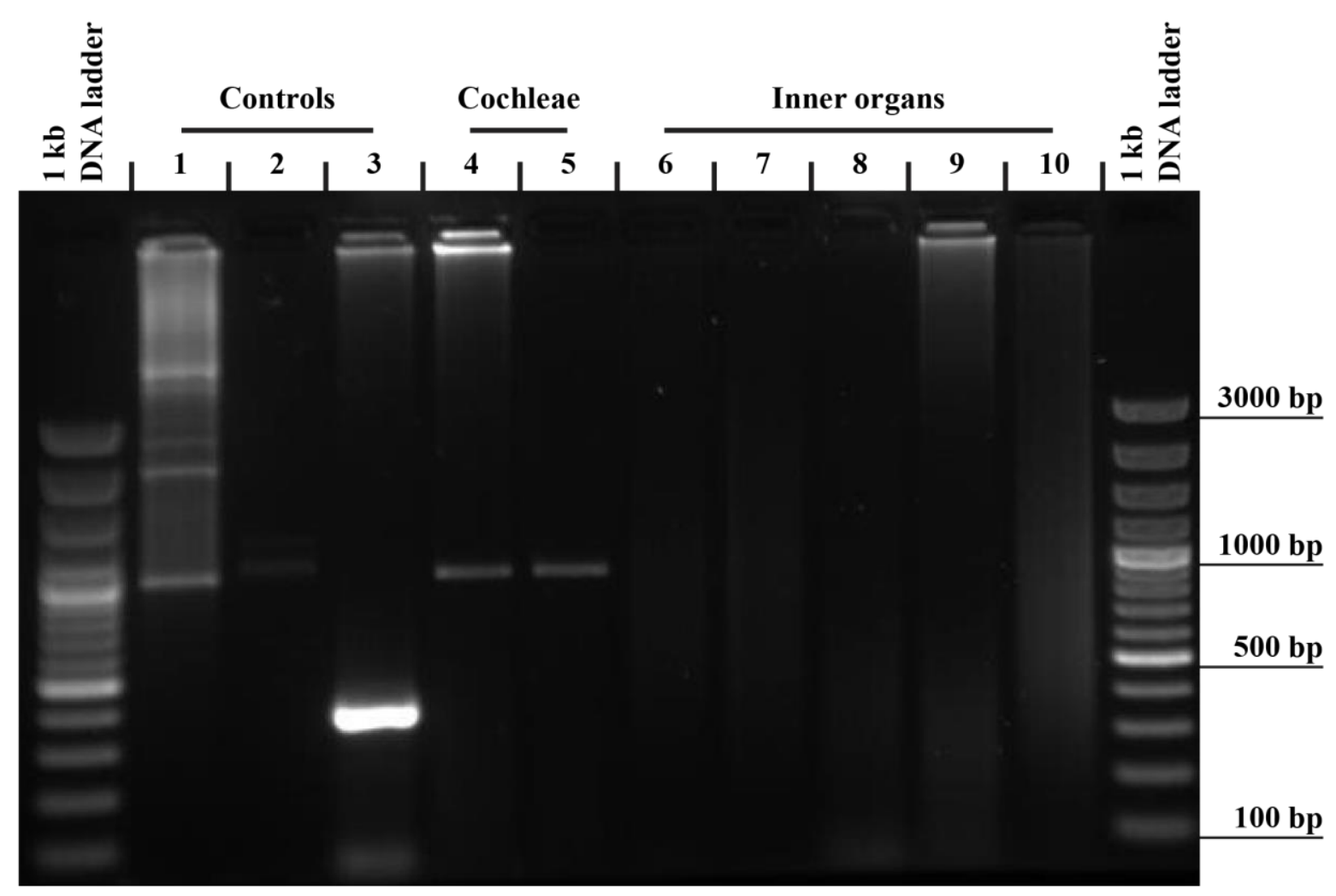

\section{Figure3.6: f-Chrimson DNA is locally restricted}

Agarose gel image of PCR products amplified from template plasmid DNA (pAAV2-hSyn-fChrimson-EYFP-WPRE-bGH) as positive control for amplified site (1), double-distilled $\mathrm{H}_{2} \mathrm{O}$ as PCR negative control (2), tissue DNA sample extracted from contralateral non-injected cochlea as DNA isolation control (3), tissue DNA samples extracted from contralateral noninjected cochlea (4), injected cochlea (5), heart (6), lung (7), liver (8), kidney (9), and spleen (10) is shown. All tissue samples are from a 1-month-old transduced mouse. Expected PCR product size for cochleae and the inner organs is $917 \mathrm{bp}(4-10)$ whereas it is $367 \mathrm{bp}$ for DNA isolation control (3). 


\section{Discussion}

In this chapter, the aim was to investigate stable, efficient, specific and safe transduction of mouse SGNs using viral f-Chrimson construct. We achieved this via unilateral injections of AAV2/6 - hSyn-f-Chrimson-YFP construct through the cochlear RWM of postnatal animals. Expression of the f-Chrimson construct lasted up to two years, average lifetime of a laboratory mouse (Flurkey, Currer, Harrison, 2016). Although the number of transduced SGNs dropped comparing the youngest and the oldest animals, from about $60 \%$ to $40 \%$ on average, the transduction level was stable for one year. Interestingly, there was a sharp decrease in transduction level of 3-month-old group along with high variability which is possibly stemming from an injection failure for that group.

I also compared the SGN densities among the groups since viable and healthy SGNs are quintessential for hearing through cochlear implant technologies. Hence, SGN cell body densities were analyzed which showed first an age-related neuron loss in both cochleae of virally transduced groups. For the injected ear, this loss was clear at the age of 12 months compared to internal non-injected counterpart. On the other hand, compared to cochleae from naïve animals at the same age, SGN density of the injected side was similar, yet with a tendency to be lower. Due to an inherent mutation in cadherin-23 gene, C57Bl/6J mouse strain is known to have sensorineural hearing loss starting from 6 months of age at highfrequency sound coding basal cochlea where hair cells die (Kane et al., 2012). Following this hair cell loss, at the age of 12 - 15 months, again from the basal part, the number of SGNs starts to diminish (Someya et al., 2009). Taken this into consideration, aging seems to be the major factor for loss of the neurons observed and expression of the channelrhodopsin might be slightly accelerating this process. Lastly, it should be also noted that the pressure injection as the delivery method seems to not cause any SGN loss.

Local delivery of the viral construct in was not limited to the injected cochlea. First clear nontarget tissue was the cochlea of contralateral side showing minimal expression. Such spread was first reported to occur in adult guinea pigs injected through RWM into the perilymph and the potential route was suspected as cochlear aqueduct connecting two cochleae (Kho et al., 2000). A recent study in mice investigates the role of cochlear aqueduct using dye tracking techniques in adult and postnatal animals (Talaei et al., 2019). There, authors observed that irrespective of age of the animals, injections through RWM resulted in distribution of the dye in brain. They hypothesize that injected compound through the round window into the scala tympani would end up in the cranial cavity by the way of cochlear aqueduct due to proximity 
of these two structures. In our injection method, this could be the route as well allowing viral suspension to reach even at the very anterior parts of the brain.

PCR-based analysis of potential spread to even distal parts of body showed that the tested inner organs are free from the viral f-Chrimson construct. In the same study (Kho et al. 2000), they have also not detected any spread to the inner organs. Using ethidium bromide labeling of DNA, authors determined a detection limit of 100 viral particles, hence 100 genomic copies of viral DNA, on agarose gel. This then would require more material to start with in case of DNA extracted from varying amount of tissue samples. For example, it was possible to detect the expected PCR product in the injected cochlea, yet it was not the case for the contralateral non-injected one. The reason why even in cochlea detection of viral DNA is not consistent as the target organ is that tissue sample from cochlea for PCR assay was collected after enough number of cryosections for histology was reached. Possibly the starting material varied substantially for cochlea samples. There was enough tissue provided for the inner organs and hence if there was even a small amount of viral existence in the inner organs this PCR detection should have yielded the target DNA sequence from the f-Chrimson construct. Lastly, RBP-2 amplification would not also refute this idea because RBP-2 gene is ubiquitous; therefore, such a comparison would not be suitable.

All in all, this study shows that expression of f-Chrimson in the auditory nerve endures for the lifetime of a mouse with minimal loss of SGNs. There was a spread of the construct up to brain without any immunological reactions. For future translational studies, for example in non-human primates, this long-term and relatively stable expression with almost no adverse effects can make chronic experiments possible using oCIs. 


\section{General Discussion}

Development of eCIs in restoring auditory function is a prominent achievement in neuroprosthetics. The device encodes sound information directly at the auditory nerve via electric pulses. However, ionic milieu of the cochlea makes it difficult to place-code of sound in a refined fashion since electric current spreads broadly. On the contrary, as light can be conveniently focused, better spectral resolution of sound can be attained along the tonotopic axis of cochlea. This could improve quality of hearing, for example, by providing better pitch perception and thereby facilitating music appreciation. Aside from the endeavor for the development of micro-engineered light stimulators, the other challenge lies within the nature of auditory nerve as it does not sense light. Here, cochlear optogenetics emerges and offers the option to render the SGNs light sensitive. This thesis revolves around the idea of optogenetic manipulation of SGNs in the aspects of physiological and safety-related functionality, compatibility and durability. Moreover, it presents three channelrhodopsins, Chronos, f-Chrimson and vf-Chrimson, which were all optimized for ultrafast control of auditory nerve. I would like to discuss these three options for future clinical or research framework.

First off, besides place-code of sound, spiral ganglion neurons fire at high rates with high fidelity to encode temporal features of soundwaves. The work has done in CatCh-injected gerbils showed that light can indeed activate narrower spectral ranges accurately compared to electric stimulation (Dieter et al, 2019; 2020a). Yet, CatCh has slow closing time constant which does not allow SGNs follow stimulation rates above $100 \mathrm{~Hz}$ with a reasonable precision where steady-state firing rate of these neurons is around 200-300 spikes per second (Wrobel et al, 2018; Liberman, 1978). Our oSU data sets using the three ultrafast opsins indicate that it is possible to trigger spike trains at stimulation rates $\sim 300 \mathrm{~Hz}$ in a highly synchronous manner. Yet, we do not manage to stimulate at higher repetition rates with high precision. With f-Chrimson, this makes sense considering its closing time constant of $3.2 \mathrm{~ms}$ at body temperature. But with faster opsins, being Chronos and vf-Chrimson, it does not fully explain the situation since their closing time constants are $0.76 \mathrm{~ms}$ and $1.6 \mathrm{~ms}$, respectively. One explanation can be that in case of Chrimson variants, $\mathrm{f}-\mathrm{Chrimson}$ has around $32 \mathrm{pA} / \mathrm{pF}$ current density under stationary illumination $(500 \mathrm{~ms})$ in $\mathrm{NG}$ cells whereas vf-Chrimson can only elicit around $8 \mathrm{pA} / \mathrm{pF}$ photocurrent density although closing kinetics is just 2-times faster in the latter. Similar pattern is also observed under pulsatile light conditions ( $3 \mathrm{~ms})$ in NG cells where current-voltage curves indicate that f-Chrimson, for example at $-80 \mathrm{mV}$ 
membrane potential, can elicit photocurrents of around 1400 pA but vf-Chrimson can only about $400 \mathrm{pA}$. So, charge exchange ratio is not directly proportional to the closing kinetics in this comparison. Therefore, to be able to compensate for the reduced photocurrent density and hence precise temporal excitability of SGNs, enhanced membrane expression is essential.

As a second speculative argument what one could also ponder that vf-Chrimson remains longer in the inactive state longer than f-Chrimson; hence renders the SGN still inexcitable. In a modeling study (Gupta et al, 2019), authors suggest a four-state photocycle (two open and two closed) for vf-Chrimson taking photoisomerization of retinal molecule into account. There, a certain portion of vf-Chrimson molecules are thought to undergo closed state 2 which does not accept photons and can only thermally relax in minutes. Lesser light-sensitivity of vf-Chrimson compared to f-Chrimson might explain this such that if more light in a certain time window is required to activate and open vf-Chrimson channels via the conformational change in the retinal molecule, it could require longer time for retinal to relax back to light accepting state. Since we also conduct stimulation rate experiments at saturating light conditions, maybe most of the vf-Chrimson is pushed to this closed state 2. Therefore, it can make only a number of membrane-embedded vf-Chrimson to pass photocurrents.

Lastly, similar firing rates with similar fidelities can point that this is irrelevant to the opsin but dependent simply on the intrinsic firing properties of the SGNs. In a study done in mouse pups (Adamson et al, 2002), authors checks the firing properties where they discover that low frequency coding apical SGNs showed longer latencies and slowly adapting responses. More basally located high frequency sound encoding SGNs, on the other hand, displayed opposite response properties. They reason such a difference in firing patterns between apical and basal SGNs due to different compositions of subunits of voltage-gated potassium channels. In our measurements, since the sample number of optically-evoked single units is generally limited to several dozens, perhaps we miss the majority of intrinsically fast-spiking SGN fibers. This can be also supported by oABR recordings, which is the measure of synchronous population response of SGNs, where sizable wave I amplitudes can be recorded up to $500 \mathrm{~Hz}$ for vfChrimson and up to $1000 \mathrm{~Hz}$ stimulation rate for Chonos. To sum this part, in terms of temporal properties of cochlear optogenetics, these three opsins are inseparable so far.

If all three opsins appear to provide equally fast temporal control of SGNs, then one can ask which one to investigate in depth for future oCI applications. Briefly, we could then question the light-related requirements of the opsins firstly as the wavelength and minimum intensity 
for neural activation. Chronos can be opted out due to its blue-light activation spectrum which would exacerbate SGN health for long-term use. Furthermore, in spite of the enhancements done for elevated membrane expression levels, light intensity thresholds are still high. Nevertheless, being a naturally-occurring ultrafast opsin and readily available multichannel blue-light oCIs make Chronos appealing for immediate research questions.

This leaves us with the red-shifted opsins, i.e. f- and vf-Chrimson. Both are activated by redlight yet the latter, even the membrane-enhanced version, require more light flux to drive auditory nerve compared to the former. Furthermore, f-Chrimson was able to induce approximately five-fold greater oABR wave I amplitudes compared to vf-Chrimson suggesting that it can recruit more SGN populations at an improved synchronicity.

In conclusion, our current understanding of ultrafast channelrhodopsins for cochlear optogenetics applications implies that it would be possible to precisely code for temporal features of sound by light. This would then advance artificial hearing and approximate auditory percepts more to the normal hearing. 


\section{References}

Adamson, C. L., Reid, M. A., Mo, Z. L., Bowne-English, J., \& Davis, R. L. (2002). Firing features and potassium channel content of murine spiral ganglion neurons vary with cochlear location. The Journal of comparative neurology, 447(4), 331-350.

Ashmore J (2008) Cochlear outer hair cell motility. Physiol Rev 88: 173 - 210

Arenkiel, B.R., Peca, J., Davison, I.G., Feliciano, C., Deisseroth, K., Augustine, G.J.,Ehlers, M.D., Feng, G., (2007). In vivo light-induced activation of neural circuitryin transgenic mice expressing channelrhodopsin-2. Neuron 54, 205-218

von Békésy G, Wever EG (1960) Experiments in hearing. New York, NY: McGraw-Hill

Boyden, E.S., Zhang, F., Bamberg, E., Nagel, G., Deisseroth, K., (2005). Millisecondtimescale,genetically targeted optical control of neural activity. Nat. Neurosci.8, $1263-1268$

Caldwell MT, Jiam NT, Limb CJ (2017) Assessment and improvement of sound quality in cochlear implant users. Laryngoscope Investig Otolaryngol 2: 119 - 124

Carricondo, F., \& Romero-Gómez, B. (2019). The Cochlear Spiral Ganglion Neurons: The Auditory Portion of the VIII Nerve. Anatomical record (Hoboken, N.J. : 2007), 302(3), 463471.

Dieter, A., Klein, E., Keppeler, D., Jablonski, L., Harczos, T., Hoch, G., Rankovic, V., Paul, O., Jeschke, M., Ruther, P., \& Moser, T. (2020). $\mu$ LED-based optical cochlear implants for spectrally selective activation of the auditory nerve. EMBO molecular medicine, 12(8), e12387.

Dieter, A., Keppeler, D., \& Moser, T. (2020). Towards the optical cochlear implant: optogenetic approaches for hearing restoration. EMBO molecular medicine, 12(4), e11618.

Dieter, A., Duque-Afonso, C. J., Rankovic, V., Jeschke, M., \& Moser, T. (2019). Near physiological spectral selectivity of cochlear optogenetics. Nature communications, 10(1), 1962.

Duebel J, Marazova K, Sahel J-A (2015) Optogenetics. Curr Opin Ophthalmol26: 226 - 232 
Dunn, W. D., Jr, Gearing, M., Park, Y., Zhang, L., Hanfelt, J., Glass, J. D., \& Gutman, D. A. (2016). Applicability of digital analysis and imaging technology in neuropathology assessment. Neuropathology : official journal of the Japanese Society of Neuropathology, 36(3), 270-282.

European Commission (2006) Directive 2006/25/EC of the European Parliament and of the Council (artificial optical radiation). Available at: http://data.europa.eu/eli/dir/2006/25/oj/eng [Accessed November 29, 2020]

Felsky D, Roostaei T, Nho K, Risacher SL, Bradshaw EM, Petyuk V, Schneider JA, Saykin A, Bennett DA, De Jager PL. Neuropathological correlates and genetic architecture of microglial activation in elderly human brain. Nat Commun. 2019 Jan 24;10(1):409.

Ferretti MT, Merlini M, Späni C, Gericke C, Schweizer N, Enzmann G, Engelhardt B, Kulic L, Suter T, Nitsch RM. T-cell brain infiltration and immature antigen-presenting cells in transgenic models of Alzheimer's disease-like cerebral amyloidosis. Brain Behav Immun. 2016 May;54:211-225.

Flurkey, K; Currer, J M.; and Harrison, D E., "Mouse models in aging research." (2007). Faculty Research 2000 - 2009. 1685.https://mouseion.jax.org/stfb2000_2009/1685

Garita-Hernandez M, Guibbal L, Toualbi L, Routet F, Chaffiol A, Winckler C, Harinquet M, Robert C, Fouquet S, Bellow S, Sahel JA, Goureau O, Duebel J, Dalkara D. (2018) Optogenetic Light Sensors in Human Retinal Organoids. Front Neurosci. Nov 2;12:789

Gauvin G, et al, 2020, Optogenetic therapy: High spatiotemporal resolution and pattern recognition compatible with vision restoration in non-human primates, BioRXiv

Gradinaru V, Zhang F, Ramakrishnan C, Mattis J, Prakash R, Diester I, Goshen I, Thompson KR, Deisseroth K (2010) Molecular and cellular approaches for diversifying and extending optogenetics. Cell 141:154-165

Gupta, N., Bansal, H., \& Roy, S. (2019). Theoretical optimization of high-frequency optogenetic spiking of red-shifted very fast-Chrimson-expressing neurons. Neurophotonics, 6(2), 025002. 
Han X, Qian X, Stern P, Chuong AS, Boyden ES. (2009) Informational lesions: optical perturbation of spike timing and neural synchrony via microbial opsin gene fusions. Front Mol Neurosci.;2:12.

Kane KL, Longo-Guess CM, Gagnon LH, Ding D, Salvi RJ, Johnson KR. Genetic background effects on age-related hearing loss associated with $\mathrm{Cdh} 23$ variants in mice. Hear Res. 2012;283(1-2):80-88.

Keppeler D, Schwaerzle M, Harczos T, Jablonski L, Dieter A, Wolf B, Ayub S, Vogl C, Wrobel C, Hoch G, Abdellatif K, Jeschke M, Rankovic V, Paul O, Ruther P, Moser T. (2020) Multichannel optogenetic stimulation of the auditory pathway using microfabricated LED cochlear implants in rodents. Sci Transl Med.12(553):eabb8086

Kho ST, Pettis RM, Mhatre AN, Lalwani AK. Safety of adeno-associated virus as cochlear gene transfer vector: analysis of distant spread beyond injected cochleae. Mol Ther. 2000 Oct;2(4):368-73.

Klapoetke, N.C., Murata, Y., Kim, S.S., Pulver, S.R., Birdsey-Benson, A., Cho, Y.K.,Morimoto, T.K., Chuong, A.S., Carpenter, E.J., Tian, Z., Wang, J., Xie, Y., Yan, Z.,Zhang, Y., Chow, B.Y., Surek, B., Melkonian, M., Jayaraman, V., Constantine-Paton, M., Wong, G.K.-S., Boyden, E.S., (2014). Independent optical excitation ofdistinct neural populations. Nat. Methods 11, 338-346

Kleinlogel S, Vogl C, Jeschke M, Neef J, Moser T. (2020) Emerging Approaches for Restoration of Hearing and Vision. Physiol Rev.;100(4):1467-1525.

Jablonski L, et al., 2020, Hearing restoration by a low-weight power-efficient multichannel optogenetic cochlear implant system, BioRXiv

Jeschke M, Moser T. (2015) Considering optogenetic stimulation for cochlear implants. Hear Res.;322:224-234.

Liberman M. C. (1978). Auditory-nerve response from cats raised in a low-noise chamber. The Journal of the Acoustical Society of America, 63(2), 442-455.

Lin JY. (2011) A user's guide to channelrhodopsin variants: features, limitations and future developments. Exp Physiol.;96(1):19-25. 
Miller CA, Abbas PJ, Robinson BK, Nourski KV, Zhang F, Jeng FC. (2006) Electrical excitation of the acoustically sensitive auditory nerve: single-fiber responses to electric pulse trains. J Assoc Res Otolaryngol.;7(3):195-210.

Munsell, Albert H. (1905). A Color Notation. Boston: G. H. Ellis Co.

Nidetz, N. F., McGee, M. C., Tse, L. V., Li, C., Cong, L., Li, Y., \& Huang, W. (2020). Adeno-associated viral vector-mediated immune responses: Understanding barriers to gene delivery. Pharmacology \& therapeutics, 207, 107453.

Rodrigues, G. A., Shalaev, E., Karami, T. K., Cunningham, J., Slater, N., \& Rivers, H. M. (2018). Pharmaceutical Development of AAV-Based Gene Therapy Products for the Eye. Pharmaceutical research, 36(2), 29.

Smith ZM, Delgutte B, Oxenham AJ. (2002) Chimaeric sounds reveal dichotomies in auditory perception. Nature;416:87-90.

Sengupta A, Chaffiol A, Macé E, Caplette R, Desrosiers M, Lampic M, Forster V,Marre O, Lin JY, Sahel J-A et al (2016) Red-shifted channelrhodopsinstimulation restores light responses in blind mice, macaque retina, andhuman retina. EMBO Mol Med 8: 1248 - 1264

Shannon RV (1983) Multichannel electrical stimulation of the auditory nerve in man. II. Channel interaction. Hear Res 12: $1-16$

Shannon RV, Cruz RJ, Galvin JJ (2011) 3rd. Effect of stimulation rate on cochlear implant users' phoneme, word and sentence recognition in quiet and in noise. Audiol Neurootol.;16(2):113-23.

Someya S, Xu J, Kondo K, Ding D, Salvi RJ, Yamasoba T, Rabinovitch PS, Weindruch R, Leeuwenburgh C, Tanokura M, Prolla TA. (2009) Age-related hearing loss in C57BL/6J mice is mediated by Bak-dependent mitochondrial apoptosis. Proc Natl Acad Sci U S A.;106(46):19432-7.

Sugano E, Isago H, Wang Z, Murayama N, Tamai M, Tomita H. (2011) Immune responses to adeno-associated virus type 2 encoding channelrhodopsin- 2 in a genetically blind rat model for gene therapy. Gene Ther.;18(3):266-74. 
Taberner, A. M., \& Liberman, M. C. (2005). Response properties of single auditory nerve fibers in the mouse. Journal of neurophysiology, 93(1), 557-569.

Talaei S, Schnee ME, Aaron KA, Ricci AJ. (2019) Dye Tracking Following Posterior Semicircular Canal or Round Window Membrane Injections Suggests a Role for the Cochlea Aqueduct in Modulating Distribution. Front Cell Neurosci.;13:471.

Wrobel, C., Dieter, A., Huet, A., Keppeler, D., Duque-Afonso, C. J., Vogl, C., Hoch, G., Jeschke, M., \& Moser, T. (2018). Optogenetic stimulation of cochlear neurons activates the auditory pathway and restores auditory-driven behavior in deaf adult gerbils. Science translational medicine, 10(449), eaao0540. 


\section{List of Abbreviations}

AAV: Adeno-associated virus

ABR: Auditory brainstem response

aABR: Acoustically-evoked auditory brainstem response

AN: Auditory nerve

ANF: Auditory nerve fiber

AP: Action potential

CD3: cluster of differentiation 3

ChR: Channelrhodopsin

CI: Cochlear implant

eCI: Electric cochlear implant

ES: Endoplasmic reticulum export signal

F-Chrimson: Fast-Chrimson

IBA1: ionized calcium binding adaptor molecule 1

IHC: Inner hair cell

oABR: Optically-evoked auditory brainstem response

oCI: Optical cochlear implant

OHC: Outer hair cell

SGN: Spiral Ganglion Neuron

TS: Membrane trafficking signal

Vf-Chrimson: Very-fast-Chrimson

YFP: Yellow fluorescent protein 


\section{Acknowledgments}

First of all, I would like to thank Prof. Dr. Tobias Moser for giving me a chance to work on this exciting project. He allowed me to be included in different aspects of this giant project. I am especially thankful to him for his encouraging attitude under the stressful conditions of pandemic and thesis. Here, I am also thankful to Prof. Dr. Manuela Schmidt and Dr. Jens Gruber for co-steering my work.

I would like to thank Dr. Vladan Rankovic for being my direct supervisor, for producing all these various viral constructs and for injecting mice. He was a backbone of this project. I would like to deliver my gratitude to Dr. David Lopez de la Morena who taught me how to conduct the physiological recordings and introduced me to understand the data. My thanks can extend to Christiane Senger-Freitag, Daniela Gerke and Sandra Gerke for their excellent technical support. For her administrative support, I also thank Patricia Räke-Kügler. I would also like to convey my gratitude to Sandra Drube, Jonas Barth and Franziska Kühne of the IMPRS Neuroscience office for their support all these years.

I feel deeply grateful to Dr. Lukasz Jablonski whose doors are always open for anyone at any time. For me and for this work, he created an understanding communication environment which made it possible to delve into questions over and over again if necessary. He also brought me in gaining insight into the optics and electronics.

Such a great gratitude, I also feel for Antoine Huet, $\mathrm{PhD}$, who created a safe environment for any question. His questioning nature, even for the basics, encourages me to utter my point of view. He taught me how to approach the electrophysiology data. I especially thank him for initiating our regular "Cake Meeting" where I am able to openly discuss my projects. Here, I would like to also thank Aida Garrido, PhD, Anupriya Thirumalai and my single-unit colleague Artur Mittring for their feedback during those meetings.

I would like to thank to Dr. Eva Gruber-Dujardin very much for her invaluable contribution to the biosafety project from hands-on work to understanding and interpreting the data. I look up to her for her professional and personal attitude. I would also like to thank Nadine Schminke and Larissa Hummel for their technical assistance.

Without support and love of friends, this work would not be possible. I thank from the bottom of my heart to Heba Ali, Büsra Coban, Tugce Isik, Günes Birdal, Sinem Sertel, Haiko Poppinga, Nicolas Lemus Diaz, Anita Krisko, Özge Demet Özcete, Zahide Ürk, Bugra Aktan, 
Serdar Capar, Lakshay Khurana and again to Lukasz Jablonski. I am just wordless now but I hope I reciprocate with you.

Last and most, I am intensely grateful and forever indebted to my mom and dad. They experience genuinely all peaks and valleys of this journey with me. I thank to my brother for his support and reminding me to step back and see the bigger picture. 